\title{
The Metabolism of Anabolic Agents In the Racing Greyhound
}

\author{
A thesis submitted in partial fulfilment \\ of the requirements for the Degree of \\ Doctor of Philosophy
}

by

Mr. Keith Robert Williams, B.Sc.

July 1999

Department of Forensic Medicine \& Science

University of Glasgow

Copyright (C) 1999 by Keith R. Williams. All rights reserved. No part of this thesis may be reproduced in any forms or by any means without the written permission of the author. 
ProQuest Number: 13833925

All rights reserved

INFORMATION TO ALL USERS

The quality of this reproduction is dependent upon the quality of the copy submitted.

In the unlikely event that the author did not send a complete manuscript and there are missing pages, these will be noted. Also, if material had to be removed, a note will indicate the deletion.

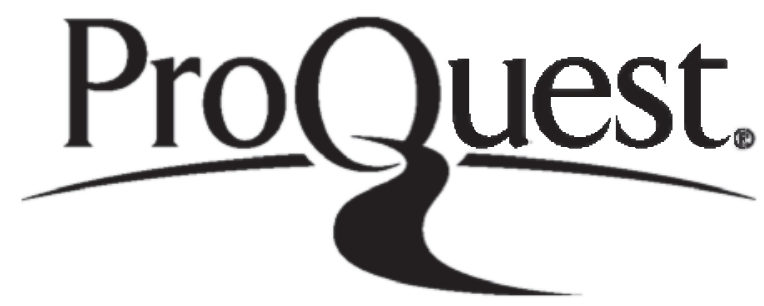

ProQuest 13833925

Published by ProQuest LLC (2019). Copyright of the Dissertation is held by the Author.

All rights reserved.

This work is protected against unauthorized copying under Title 17, United States Code Microform Edition (C) ProQuest LLC.

ProQuest LLC.

789 East Eisenhower Parkway

P.O. Box 1346

Ann Arbor, Ml $48106-1346$ 


$$
11726(\text { copy } 1)
$$


To my parents for all their help, support and encouragement 
List of Figures $\quad$ V

List of Tables $\quad$ VIII

Summary

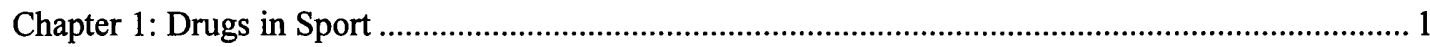

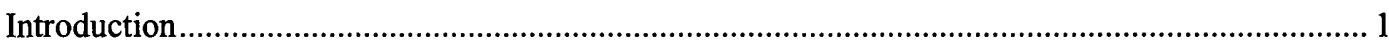

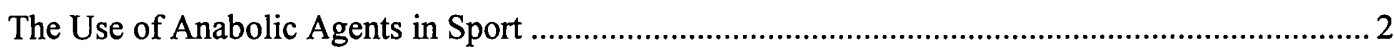

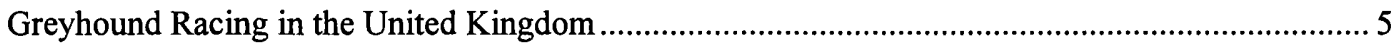

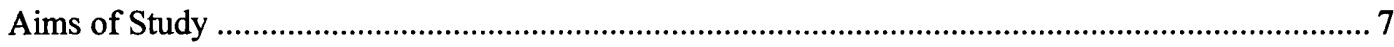

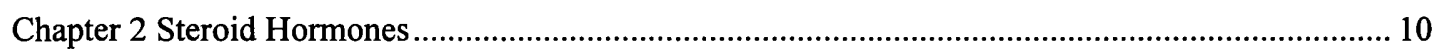

Steroid Nomenclature and Structure ...................................................................................... 10

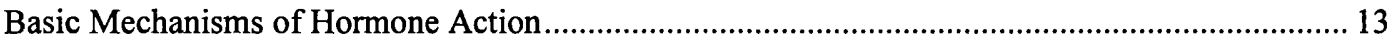

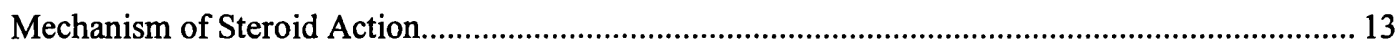

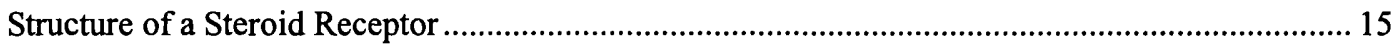

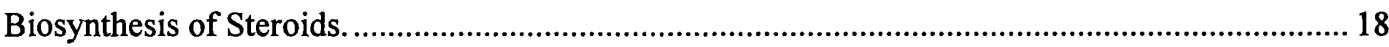

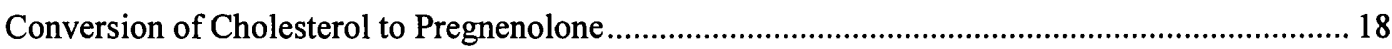

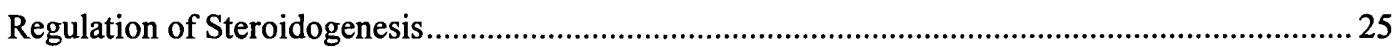

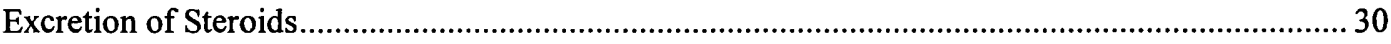

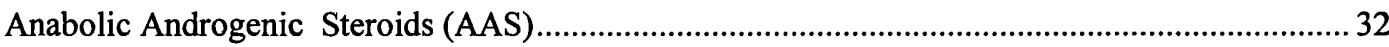

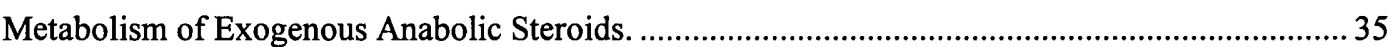

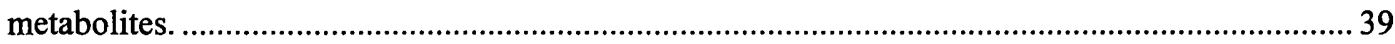

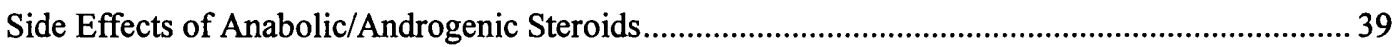

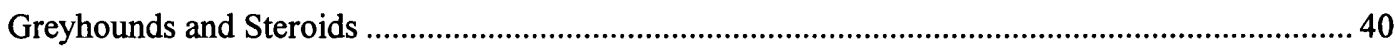

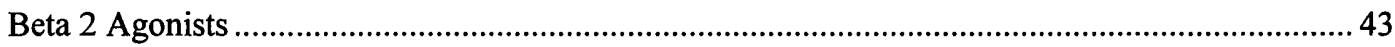

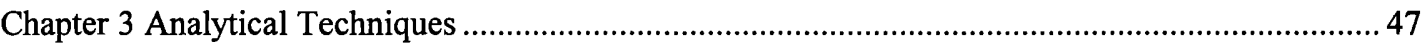

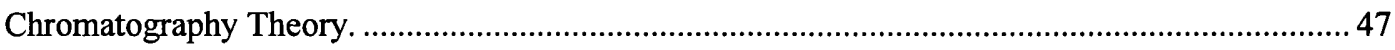

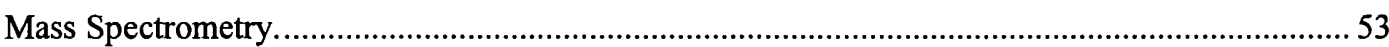

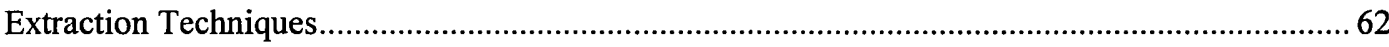

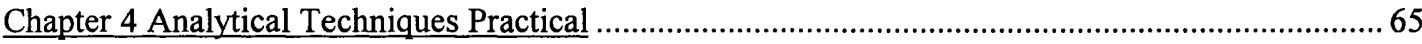


Introduction

Elucidation of the background steroid profile of the racing greyhound

glucuronide Metabolism of Testosterone in the Racing Greyhound ....................................... 92

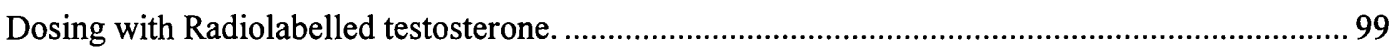

Conclusions

Chapter 6 Metabolism of Nandrolone and Boldenone .......................................................... 107

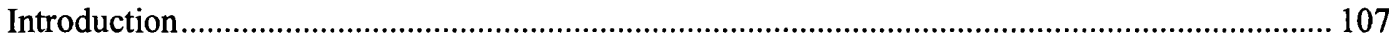

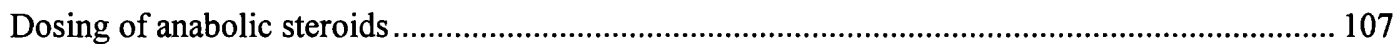

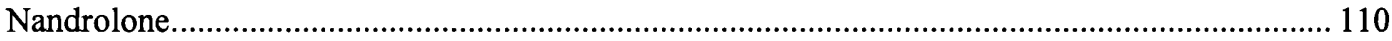

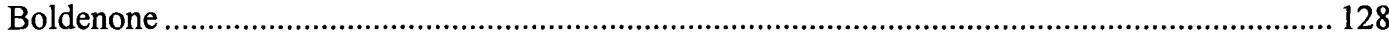

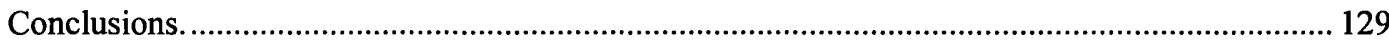

Chapter 7 The analysis of clenbuterol in the plasma and urine of the racing greyhound. ................. 130

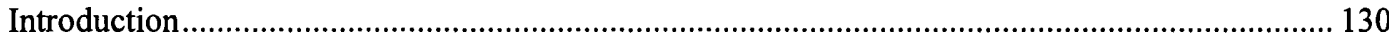

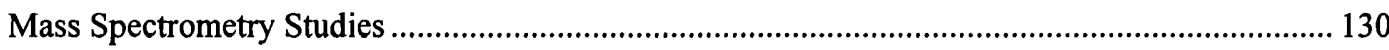

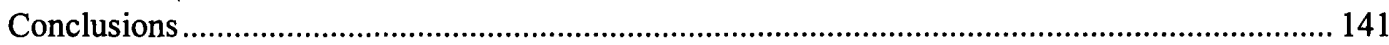

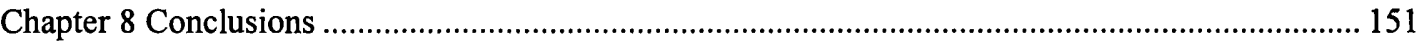

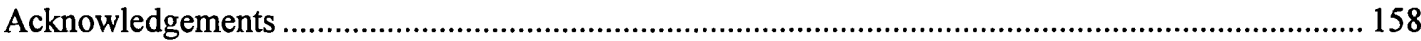

References

Appendix 1 Paper published in Analytica Chimica Acta

Appendix 2 Two papers presented at the international association of Forensic Science, Dusseldorf, August 1993

Appendix 3 Greyhounds used in metabolism studies 


\section{List of Figures}

Figure 2.1 a Basic Steroid Hydrocarbon Skeleton 11

Figure $2.1 \mathrm{~b}$ Numbering System of Steroid Rings 11

Figure 2.3 Proposed Mechanisms for the Action of Hormones $\quad 14$

$\begin{array}{ll}\text { Figure 2.4 Mechanism of Steroid Action. } & 16\end{array}$

$\begin{array}{ll}\text { Figure 2.5 Structure of Steroid Receptor } & 17\end{array}$

Figure 2.6 Synthesis of Cholesterol from Acetyl CoA. 19

$\begin{array}{ll}\text { Synthesis of testosterone from pregnenolone } & 217\end{array}$

Figure 2.8 Biosynthesis of Corticosteroids 23

$\begin{array}{ll}\text { Figure 2.9 Biosynthesis of Oestrogens } & 24\end{array}$

Figure 2.10 The Inhibition of Steroid Synthesis by Steroids 27

Figure 2.11 Metabolism of Testosterone 29

Figure 2.12 Mode of action of Beta agonists $\quad 46$

Figure 3.1 Schematic Diagram of an Electron Impact Source. $\quad 54$

Figure 3.2 Various Types of Mass Spectrometers 56

$\begin{array}{ll}\text { Figure 3.3 Definitions of Resolution } & 60\end{array}$

Figure 5.1 Urinary steroid profile of a male racing greyhound 86

$\begin{array}{ll}\text { Figure 5.2 Urinary steroid profile of a female racing greyhound } & 87\end{array}$

Figure 5.3 Sample chromatogram showing the separation of

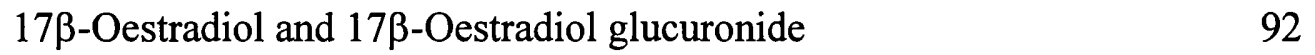

Figure 5.4a Concentration of testosterone in the urine of Dog 1 as determined by RIA 
Figure 5.4b Concentration of testosterone in the urine of Dog 2 as determined by RIA

Figure 5.5 Disintegration per minute per millilitre of plasma versus time after dosing following an intravenous injection of a greyhound with ${ }^{3} \mathrm{H}$ - testosterone

Figure 5.6: Radioactivity in urine up to 144 hours post-dose 102

Figure 5.7 Graph of the ratio between labelled and unlabelled testosterone following administration of approximately $2 \mathrm{mg}$ of deutero-labelled testosterone

Figure 6.1 Structures of Nandrolone, Nandrolone Laurate and Boldenone 109 Figure 6.2 Mass spectra of Oxime and TMS derivatives formed

Figure 6.3 Heptafluoroderivative of Nandrolone

Figure 6.4 Chemical structures of nandrolone derivatives formed.

114

Figure 6.5 Concentration of nandrolone in the plasma of Dog 1 versus time following intramuscular injection of 50 milligrammes of nandrolone laurate

Figure 6.6 Concentration of nandrolone in the plasma of Dog 2 versus time following intramuscular injection of 50 milligrammes of nandrolone laurate

Figure 6.7 Concentration of testosterone in the plasma of dog 1 following the administration of nandrolone

Figure 6.8 Testosterone:Epitestosterone ratio in plasma for dog 1 overlaid on top of concentration of testosterone in plasma for dog 1

Figure 6.9 Androsterone:etiocholanolone Ratio in the plasma of dog 2

following the administration of 50 milligrammes nandrolone laurate 
Figure 6.10 Concentration of nandrolone per milligramme of creatinine against time after dose for dog 1 dosed with 50 milligrammes of nandrolone laurate.

Figure 6.11 Concentration of creatinine versus time before and after dosing

Figure 7.1 Structures of Clenbuterol and Salbutamol

Figure 7.2 Derivatisation of Clenbuterol as Proposed by Girault et al ${ }^{100}$

Figure 7.3 Proposed Derivatisation reaction of Clenbuterol in this study

Figure 7.4 Mass Spectrum of the Trimethylsilyl derivative of Clenbuterol

Figure 7.5 Electron Impact Mass spectra of perfluoroacyl derivatives of clenbuterol

Figure 7.6 Positive Ion chemical ionisation mass spectra of perfluoroacyl derivatives of clenbuterol

Figure 7.7 Negative Ion chemical ionisation Mass spectra of perfluoroacyl derivatives of clenbuterol

Figure 7.8 Levels of clenbuterol in plasma versus time after dose for a male racing greyhound following oral administration of 2 milligrammes of clenbuterol

Figure 7.9 Levels of clenbuterol in plasma versus time after dose for a female racing greyhound following oral administration of 2 milligrammes of clenbuterol

Figure 7.10 Levels of clenbuterol in urine versus time after dose for a male racing greyhound following oral administration of 2 milligrammes of clenbuterol

Figure 7.11 Levels of clenbuterol in urine versus time after dose for a female racing greyhound following oral administration of 2 milligrammes of clenbuterol 


\section{$\underline{\underline{\text { List of Tables }}}$}

Table 1. Drugs Reported Present in the Urine of Racing Greyhounds Tested at the Reference Laboratory at the University Of Glasgow in 1988.

Table 2.1 Commonly available anabolic preparations

Table 2.2 Metabolites of Some Exogenous Steroids

Table 2.3 Side effects of anabolic/androgenic steroid use

Table 2.4 Some commercially available beta agonists

Table 4.1: Selected ion recording parameters used for the analysis of selected steroids as their trimethylsilyl derivatives.

Table 4.2: Selected ion recording parameters used for the analysis of selected steroids as their heptafluorobutyrate derivatives.

Table 4.3: Selected ion recording parameters used for the analysis of selected steroids as their trimethylsilyl derivatives.

Table 4.4: Selected Ion Recording Parameters used for the Analysis of Clenbuterol and Salbutamol as their Trimethylsilyl Derivatives

Table 5.2 Recovery of radiolabelled steroids extracted from pooled racing greyhound urine.

Table 5.3 Ions monitored for the determination of testosterone in urine.

Table 5.4 Recovery of tritiated testosterone from spiked plasma samples 


\section{Summary}

The work presented in this thesis was concerned with the detection of anabolic drugs in the racing greyhound. The use of these substances in racing animals is prohibited by the National Greyhound Racing Club in the rules governing the sport. Prior to this study, little information existed regarding the metabolism of these anabolic agents in the racing greyhound or the detection of their administration.

This study initially established a greyhound metabolic unit and developed an experimental protocol based on fully trained racing greyhounds as the animal model. The unit and protocol were licenced by the Home Office for this purpose. Because of the lack of information in the literature, work was carried out to establish the normal urinary steroid profile in the racing greyhound and some basic information regarding steroid metabolism in the racing greyhound. Subsequently, the metabolism of selected anabolic steroids (nandrolone and boldenone) in the greyhound was studied, together with the effects of these exogenous steroids on the normal steriodal profile. Finally, the metabolism in the greyhound of another anabolic agent, clenbuterol, was examined.

Methodology was developed for the analysis of steroids in urine and plasma based on solid phase extraction, enzymatic hydrolysis, Sephadex LH-20 fractionation and qualitative and quantitative analysis by gas chromatography-mass spectrometry (GCMS). The extraction protocols were assessed using radiolabelled steroids and found to be acceptable. Hydrolysis procedures were assessed by HPLC and found to be 
satisfactory. A variety of derivatisation reagents were evaluated to optimise the GC-MS procedure .

It was not possible to detect the presence of steroids in the urine of male greyhounds using full scan mass spectrometry. In female greyhounds, however, it was possible to identify the presence of a pregnane, an estrogen and two corticosteroids.

Additional constituents were present which may also have been steroids but their identification could not be confirmed. Testosterone was shown to be present in greyhound urine using radioimmunoassay. Maximum concentrations of testosterone measured were approximately $25 \mathrm{ng} / \mathrm{mg}$ creatinine and $10 \mathrm{ng} / \mathrm{mg}$ creatinine for two male greyhounds.

The metabolism and turnover of testosterone in the greyhound were investigated using labelled forms of the drug. Radiolabelled testosterone was administered to a male greyhound at a low dose, designed to increase the metabolic pool size of testosterone in the animal as little as possible. Plasma samples and complete urine collections were obtained. Elimination of the radiolabelled material from the plasma was rapid and approximately $40 \%$ of the dose was recovered in the urine by 7 days post dose. An apparent volume of distribution of 5.8 litres was calculated for testosterone.

A quantitatively significant dose of deuterium labelled testosterone ( 2 milligrams) was administered intravenously to a greyhound. The testosterone pool size for the animal was estimated by isotopic dilution at approximately $190 \mu \mathrm{g}$. 
Two exogenous anabolic steroids (nandrolone and boldenone) were administered intramuscularly to two male racing greyhounds. Nandrolone was found to be present in predose urine and plasma samples indicating that nandrolone is in fact an endogenous steroid in the racing greyhound. Following administration of nandrolone to the two male dogs, levels of nandrolone in plasma rose from 30 and $60 \mathrm{ng} / \mathrm{ml}$ to over $100 \mathrm{ng} / \mathrm{ml}$ in both dogs. Elimination of nandrolone after a few days brought the plasma concentration back to the initial starting levels. Levels of endogenous steroids were depressed in plasma samples along with the concentration of creatinine excreted in the urine.

Boldenone was not detected in either plasma or urine samples following intramuscular administration. While depression of the endogenous profile did occur, it was less than that which occurred following dosing with nandrolone.

Clenbuterol is a beta-agonist drug that has been abused as an anabolic agent both in human sport and in livestock farming. Methodology was developed for the detection of clenbuterol in plasma and urine samples from the racing greyhound. Although the greatest sensitivity could be achieved using negative ion chemical ionisation-mass spectrometry in association with a heptafluorobutyrate derivative, the use of a simpler trimethylsilyl derivative and electron impact ionisation was found to be adequate and was considered more appropriate as a routine method. Clenbuterol was administered orally to one male and one female racing greyhound. Severe side effects were noted within 2 hours post dose and continued past 24 hours post dose. Peak plasma concentrations of clenbuterol occurred between 5 - 24 hours post dose 
and it was possible to determine the presence of clenbuterol in plasma and urine of the dogs for at least 8 days post dose.

It was concluded that the principal aims of the project had been achieved. 


\section{Chapter 1: Drugs in Sport}

\section{Introduction}

With the rewards for success in sporting events currently being so high, it is not surprising that competitors will do almost anything to gain an advantage over their opponents. To try and gain this advantage, individuals may roughen up one side of a cricket ball in an effort to increase the swing of the ball to catch out an unwary batsman, insist a game of chess is played on a board with green and red squares to try and disrupt the opponent's concentration, as Bobby Fischer did in 1972 , or use chemical substances to allow an individual to gain an unfair physical advantage over other competitors. In the latter case, a person may take substances for short-term effects, for example sedatives, which can be used to reduce nerves or anxiety in snooker and archery, or use drugs, which are intended to give long-term benefits such as anabolic agents.

The use of chemical substances in an effort to gain an advantage is not a modern phenomenon. In the Third Century BC, Greek athletes ate hallucinogenic mushrooms ${ }^{1}$. Viking warriors used root extracts to induce berserker trances. In the last century in 1879, competitors in six-day bicycle races used caffeine, alcohol, nitro-glycerine, ether dropped on sugar cubes, strychnine pills, cocaine and opium. The Olympic marathon champions in both 1904 and 1908 had almost certainly taken strychnine.

In 1960, Knud Jensen of Denmark, a cyclist, died on the opening day of the Rome Olympics. Traces of ronicol (a vasodilator) and amphetamine were found in his body at the post-mortem examination. In 1967, the British cyclist Tommy Simpson died while competing in the 'Tour de France'. Like Jensen 7 years earlier, amphetamine was found in his body at the post-mortem. These tragedies and others led to the introduction of legislation in various countries and the introduction of dope testing for both Olympic events in 1968, the Winter Games at Grenoble and the 
summer games at Mexico City. This legislation and dope testing has not, however, eliminated the use of drugs in sport.

\section{The Use of Anabolic Agents in Sport}

In 1973, Howard Connelly, the Olympic hammer champion in 1956 stated:'the overall majority of athletes I know would do anything and take anything short of killing themselves to improve their athletic performance.'

To improve strength and muscle bulk, and consequently to improve their athletic ability, some individuals have used anabolic agents.

The effects of testosterone were first noted in the eighteenth century, when John Hall transplanted the testes of a cock to hen. The hen developed male characteristics. In 1849, Berthold showed that implanting gonads into castrated roosters could prevent the effects of castration. Various 'magic potions' then became available over the next 75 to 100 years and were primarily sold on the basis that they could help prevent ageing and maintain sexual potency. These potions ranged from injecting an aqueous suspension of guinea pig testicles to the transplantation of monkey testicles into human males in the 1920's.

It was in 1930 that one of the first androgens was isolated. Androstenedione $(15 \mathrm{mg}$ ) was isolated from 15,000 litres of urine. Soon after, testosterone was isolated and eventually synthesised in 1935 . Experiments continued with testosterone throughout World War Two. It was given to German soldiers to increase their aggression and, ironically, to victims of the concentration camps to increase their strength and muscle bulk. Sporting use of steroids probably originated in the 1950's. Dr John B Zeigler, the US weightlifting team doctor for the world championships in Vienna in 1954 claimed that the Russian team doctor told him that certain members of the Russian team were taking synthetic testosterone ${ }^{2}$. Dr Zeiger started to use dianabol, a synthetic oral steroid developed by Ciba Geigy with a high anabolic/androgenic ratio ${ }^{3}$ with weightlifters but soon discontinued his involvement with steroids when he started to notice side effects in users such as the shrinking of 
testicles ${ }^{2}$. The use and experimentation with anabolic steroids continued throughout the 1960's. It was predicted by the Scottish Athlete David Jenkins that five of the top men in every sport would be using anabolic steroids by the Montreal Olympics ${ }^{3}$. The use of anabolic steroids was not only practised by individuals. Franke and Berendonk have published a review of the anabolic steroids used in the German Democratic Republic ${ }^{4}$.

As indicated earlier, testing for banned substances commenced in 1968 in the Olympic Games. It was not until the 1976 Olympics in Montreal that testing for anabolic steroids came into effect. The initial tests were carried out by radioimmunoassay following procedures developed by Professor Raymond Brooks of St. Thomas' Hospital, London.

Even though the use of anabolic steroids could be detected analytically in 1976, testosterone was not added to this list until 1982. A problem existed in determining the use of exogenous sources of this natural, endogenous steroid. It was noted that the ratio of the concentrations of testosterone and its inactive stereoisomer, epitestosterone (T/EpiT ratio) normally lay between 1:1 and 2.5:1 in humans. Testosterone use was said to have occurred if the T/EpiT ratio exceeded $6: 1^{5}$. The biochemical basis for this test depends on a negative feedback mechanism in the hypothalamus-pituitary-gonad axis, summarised in figure 1.1. The validity of this test has been challenged, notably in the case of the athlete Diane Modahl.

The detection of steroid use in sport is now a highly sophisticated process. The early presumptive radioimmunoassay tests have been replaced with gas chromatography-mass spectrometry, including the introduction of high resolution mass spectrometry for the 1996 Olympic Games in Atlanta, to provide the definitive answer which can stand up to scientific and legal scrutiny. Even though there is a wide variety of testing procedures in place, individuals still try to use anabolic agents to gain a competitive edge. In 1988, Ben Johnson of Canada was stripped of his Gold Medal in the $100 \mathrm{~m}$ at the Seoul Olympics after testing positive for Stanozolol (Winstrol), a 17 $\alpha$-alkylated oral steroid. It has been alleged that he thought he was 
using dehydrotestosterone, which was believed to be undetectable in 1988, but his coaches and trainers provided him with stanozolol instead. In 1992, the British athlete Jason Livingstone was tested positive for Dianabol (methandrostenolone), one of the original anabolic steroids that was no longer popular due to its serious side effects. Recent studies have demonstrated the detection of stanozolol in hair, further advancing the window of detection to the length of the hair sample ${ }^{6}$.

Athletes turned to the use of other anabolic agents in order to try and evade detection. A popular agent was clenbuterol, a $\beta$-agonist. Originally used to treat asthma, the first use of this compound as an anabolic agent is credited to British athletes in $1988^{7}$, and like all other performance enhancing drugs it was thought to be undetectable. In addition to its use in humans, it has been used illegally to increase muscle bulk in beef cattle. Very sensitive and specific methodology has been developed for detection and analysis of clenbuterol in a variety of samples ${ }^{89}$. Again, the testing procedures have caught up with individuals who use drugs and the German athlete Katrina Krabbe was tested positive for clenbuterol while racing in South Africa.

Drugs like the anabolic steroids and clenbuterol are now gradually being replaced by compounds like hGH (human growth hormone), a 191 amino acid peptide, and iGF 1 and iGF2 (insulin like growth factor 1 and 2). It is also probable that as testing procedures are developed for these compounds, individuals will switch to other alternatives.

Conventional wisdom is that anabolic agents should not be used continuously, otherwise plateauing occurs, that is, after initial gains, the agents no longer exert their effect on the body. Consequently, a series of procedures have been developed to avoid plateauing ${ }^{10}$. The agents are used in cycles of four to twelve weeks, followed by a clear period, allowing the body to re-establish its normal conditions (e.g. liver function, normal steroid profiles). During the periods of drug consumption, individuals 'stack', 'shotgun' or 'stagger' the anabolic agents. Stacking involves the use of two agents simultaneously, normally an oral steroid and 
an injectable steroid. Shotgunning involves the use of more than two agents simultaneously and staggering is the changing of the agents periodically. All these processes are designed to stop plateauing. There is little evidence to suggest that they work. A detailed description of the mode of action of steroids is given in later chapters. In addition to these procedures, many individuals use far larger doses of steroids than would be used normally, thereby increasing the risk of undesirable side effects.

\section{Greyhound Racing in the United Kingdom}

In the United Kingdom, there are two types of greyhound racing tracks. There are the tracks which are regulated by the National Greyhound Racing Club (NGRC) and the "gaff" or "flapping" tracks which in effect are independent tracks with no one body regulating the racing which occurs on these tracks. There were 38 NGRC regulated tracks in the U.K., two of which were in Scotland: Shawfield Stadium in Glasgow and Powderhall Stadium in Edinburgh (now no longer in operation). As well as the two NGRC tracks, there are up to 14 flapping tracks in Scotland, mostly around the central belt. On each type of track the overall format is similar. There are ten to twelve races in each race meeting with each race normally containing six dogs. This number is not fixed as NGRC rules allow up to eight dogs in a single race and a race may be run with less than six greyhounds in the traps.

Prior to racing, each greyhound is subjected to time trials to establish a formbook. Once this form has been established, the greyhounds are graded and placed into races according to their form. A handicap system also operates at NGRC registered tracks where a greyhound may be given up to a 10 metre start over its rivals. At flapping tracks each dog is weighed, visually inspected and earmarked prior to each race. No more detailed examination of the animal is carried out.

At NGRC regulated tracks, a more detailed veterinary examination is carried out and a urine sample is taken prior to the race to screen for the presence of any illegal doping substance. As with other sporting bodies such as the Jockey Club and 
the International Olympic Committee, the NGRC has banned the use of substances that will either enhance or diminish the performance of a racing animal. The definition of an individual who is guilty of doping a greyhound is given as follows in the NGRC rulebook, rule number $174 \mathrm{a}^{11}$ :-

[A person is liable to disciplinary action if he/she]

i. has administered or attempted to administer or has allowed or caused to be administered or connived at the administration for any improper use to a greyhound of any quantity of a substance which, by its nature, could affect the performance of a greyhound the origin of which on or in the tissues, body fluids or excreta of a greyhound could not be traced to normal or ordinary feeding or care; or

ii. has had in his/her charge a greyhound which on examination under rules 113,116 , or 173 showed the presence on or in its tissues or bodily fluids or excreta of any quantity of any substance which by its nature could affect the performance of a greyhound or which shows evidence in any way of the administration for any improper use, the origin of which cannot be traced to normal or ordinary feeding or care.

This statement covers a wide range of substances and includes caffeine and theobromine that are allowed in small quantities in other sports. For comparison, the International Olympic Committee rules provide a list of banned substances and doping methods and the Jockey Club rules state that it is illegal to administer a substance that will affect a named body system (e.g. the nervous system, the cardiovascular system).

This first urine sample is screened and tested at the trackside. If any trace of a compound is detected which contravenes the above rule, the greyhound is withdrawn from the race meeting immediately. In a further attempt to eliminate the chemical manipulation of the greyhounds, in the event of a greyhound not 
performing to its established form, a second screen is performed based on a postrace sample of blood or urine. This latter sample is sent to the reference laboratory at Newmarket, formerly the Department of Forensic Medicine and Science at the University of Glasgow, where it is analysed by radioimmunoassay (RIA), thin layer chromatography (TLC), high performance liquid chromatography (HPLC) gas chromatography (GC) and gas chromatography-mass spectrometry (GC-MS). Table 1 lists the substances found in the samples examined in the reference laboratory in 1988. These results came from 637 samples tested at the reference laboratory, with $5.81 \%$ of the samples giving positive results.

If a racing animal is found to be positive for a banned substance, the owner/trainer can be fined and his racing licence can be suspended for a period of time.

\section{Aims of Study}

To implement a successful doping control program for anabolic agents, a certain minimum level of information is required, for example the normal urinary and plasma steroid pattern in a racing greyhound, the effects of anabolic steroids on this pattern and pharmacokinetic data of the various compounds used. Very little data exists on these topics for the racing greyhound. The racing animals may display significantly different patterns of responses to anabolic agents when compared not only to other species but also to other breeds of dogs due to their physiology and training conditions e.g. little or no body fat, high protein diet, active training regime. It is necessary to establish this basic information under controlled circumstances to allow appropriate control measures to be introduced. The aims of this project could therefore be summarised as follows

1. to establish a metabolic unit for greyhounds in which the dogs would be trained in an identical manner to racing animals; 
2. to characterise the normal urinary and plasma steroid profiles for the racing greyhound;

3. to obtain some basic pharmacokinetic information regarding steroid metabolism in the racing greyhound;

4. to examine the pharmacokinetics of specific anabolic agents in the racing greyhound;

5. to examine the effects of these exogenous steroids on the normal steroid profile;

6. to examine the pharmacokinetics of other anabolic agents (namely clenbuterol) in the racing greyhound 


\begin{tabular}{|l|c|}
\hline \multicolumn{1}{|c|}{ Drug } & $\begin{array}{c}\text { Number of } \\
\text { Positive Results }\end{array}$ \\
\hline Caffeine & 13 \\
\hline Chlorpromazine & 4 \\
\hline Cyclizine & 4 \\
\hline Quinalbarbitone & 4 \\
\hline QuininelQuinidine & 3 \\
\hline Primidone and Phenobarbitone & 1 \\
\hline Amphetamines & 1 \\
\hline Ibuprofen & 1 \\
\hline Caffeine, Theobromine and Diazepam & 1 \\
\hline Millophylline & 1 \\
\hline Diazepam and Diphenhydramine & 1 \\
\hline Paracetamol & 1 \\
\hline Procaine & 1 \\
\hline Glutethimide & 1 \\
\hline Total & 37 \\
\hline
\end{tabular}

Table 1. Drugs Reported Present in the Urine of Racing Greyhounds Tested at the Reference Laboratory at the University Of Glasgow in 1988. 


\section{Chapter 2 Steroid Hormones}

\section{Steroid Nomenclature and Structure}

All steroids are based on the cyclopentaperhydrophenanthrene structure.

There are five main groups of mammalian steroids. These are the cholestanes (C27), cholanes (C24), pregnanes (C21), androstanes (C19) and oestranes (C18). The basic structures of these compounds and the numbering system employed are presented in Figure 2.1. These compounds are all derived from cholesterol. In naturally occurring steroids, these basic structures can be modified in three main ways;

i. the addition of hydroxyl groups.

ii. the addition of carbonyl groups.

iii. the introduction of unsaturation into the ring system.

The position of the modification is noted by number and prefixes and suffixes used to show the presence of various hydroxyl and carbonyl substituents. If there is more than one group which can act as a suffix, a series of rules have been established and a specific order of preference has been laid down by the International Union of Pure and Applied Chemistry (IUPAC) and the International Union of Biochemistry ${ }^{12,13}$. As well as the systematic naming system, there exists a large number of names which originated in the historical development of the field.

Stereoisomerism also commonly occurs at various key locations throughout the molecule, notably the orientation of the hydrogen atom on $\mathrm{C} 5$. When there is a hydroxyl group present within the molecule, there is the opportunity for the group to take up one of two stereochemical positions. The group can lie on either the alpha or beta side of the ring structure (Figure 2.2). This stereochemical difference can dramatically influence the physiological and biochemical effects of a particular steroid. Testosterone (17ß-hydroxy-androst-4-ene-3-one) is a potent androgen while epitestosterone (17 $\alpha$-hydroxy-androst-4-ene-3-one) is inactive. 

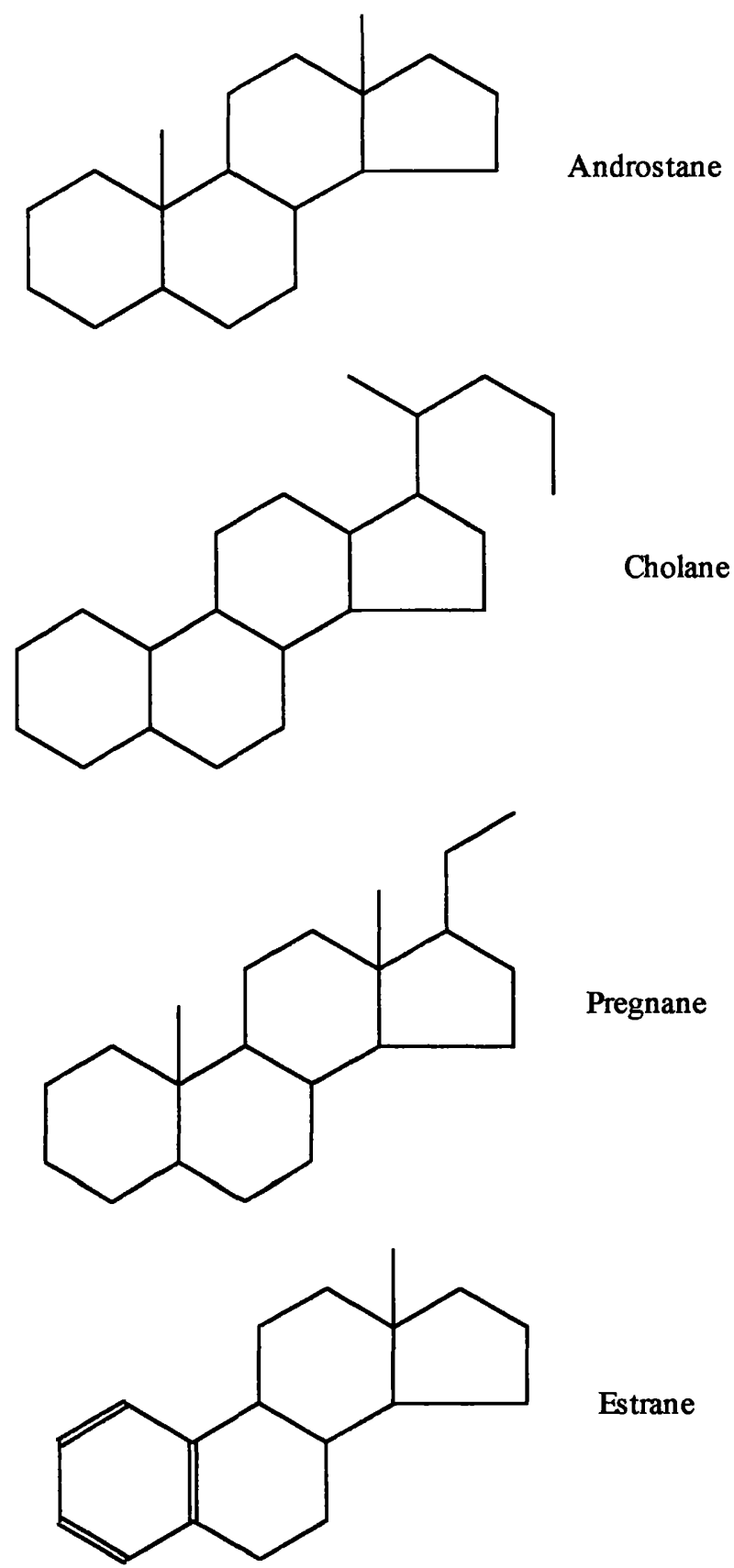

Figure 2.1a Basic Steroid Hydrocarbon Skeletons

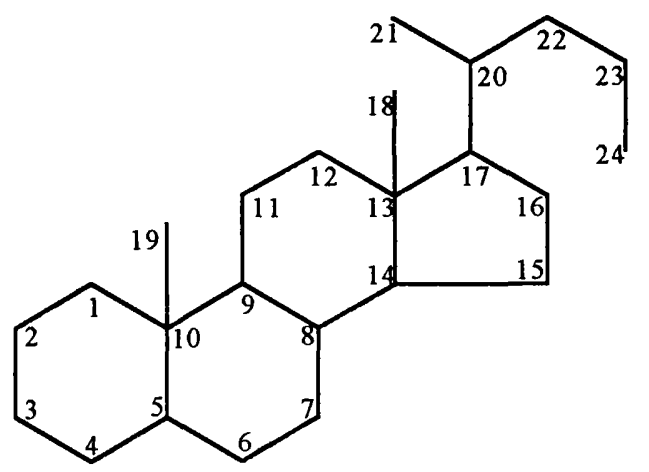

Figure 2.1b Numbering System of the Steroid Rings 


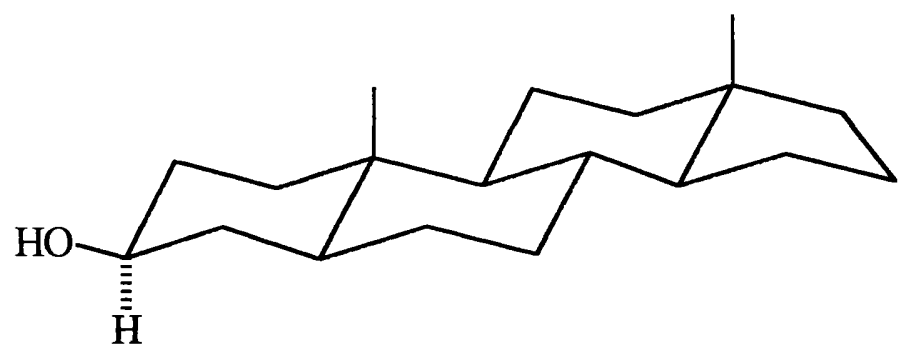

Androstan-3 $\beta$-ol

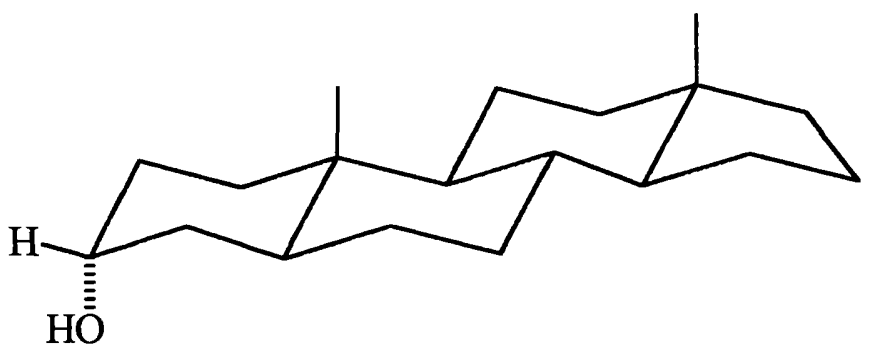

Androstan-3 $\alpha-$ ol

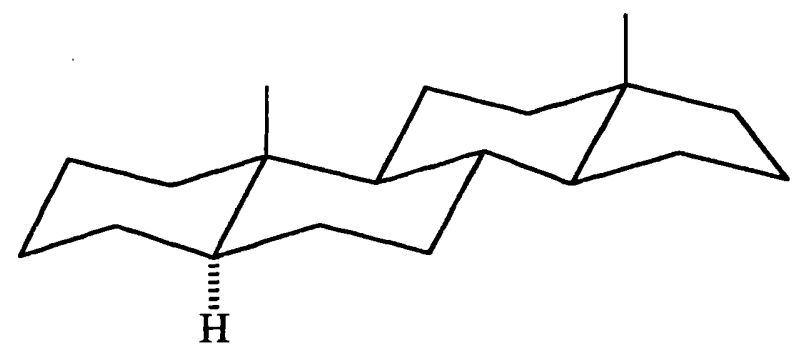

5- $\alpha$-Androstane

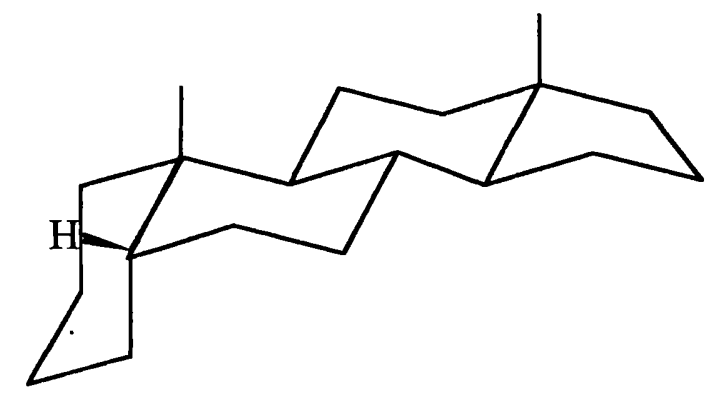

5- $\beta$-Androsane

Figure 2.2 Asymmetry in the Steroid Skeleton 


\section{Basic Mechanisms of Hormone Action}

Three possible mechanisms for the action of hormones within a biological system have been proposed ${ }^{14}$. These are illustrated by Figure 2.3

In the first mechanism, the hormone binds directly to a rate limiting enzyme, alters the conformation of the enzyme and as a result either activates or deactivates the enzyme. This was the earliest of the three mechanisms proposed. It was found to present too simplified a view of the mode of hormone action and the concept of a receptor was introduced.

Mechanism 2 involves the binding of the hormone to a receptor that in turn binds to a rate-limiting enzyme to cause the metabolic changes. This mechanism also appears to be too simple an explanation for the mode of action of hormones.

The third and most complex mechanism involves at least four components. Initially, the hormone binds to a receptor. This hormone-receptor complex (H-R) then binds to an acceptor, which in turn exerts its effect on a rate-limiting enzyme. The mechanism of steroid action is thought to follow this third model.

\section{Mechanism of Steroid Action}

The current model of steroid action is similar to the mechanism proposed by Gorska et al and Jensen et al ${ }^{15,16}$. It is described diagrammatically in Figure 2.4.

Steroids exist in the bloodstream in one of two forms, free or protein bound. When existing in the free form, they can passively diffuse through the outer cell membrane into the cytosol and pass into the nucleus of the cell. Once within the cell nucleus, the steroid can bind reversibly to a receptor protein $\left(\mathrm{K}_{\mathrm{D}}=10^{-10}\right.$ to $\left.10^{-8}\right)$ and form a stable complex. The receptor proteins have been found to be exceptionally steroid- and tissue-specific ${ }^{17}$ and there are thought to be around 10,000 to 100,000 molecules of each receptor present per cell. Once bound, the steroid causes conformational changes to the receptor which allow the steroid-receptor complex to bind reversibly to the chromatin at a site called the Steroid Response Element (SRE) 


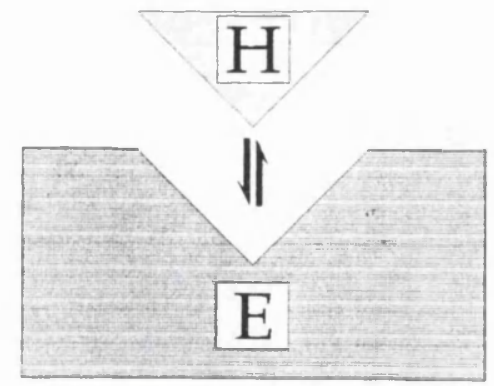

Mechanism 1

$$
\begin{aligned}
& H=\text { Hormone } \\
& R=\text { Receptor } \\
& A=\text { Acceptor } \\
& E=\text { Enzyme }
\end{aligned}
$$

Mechanism 2
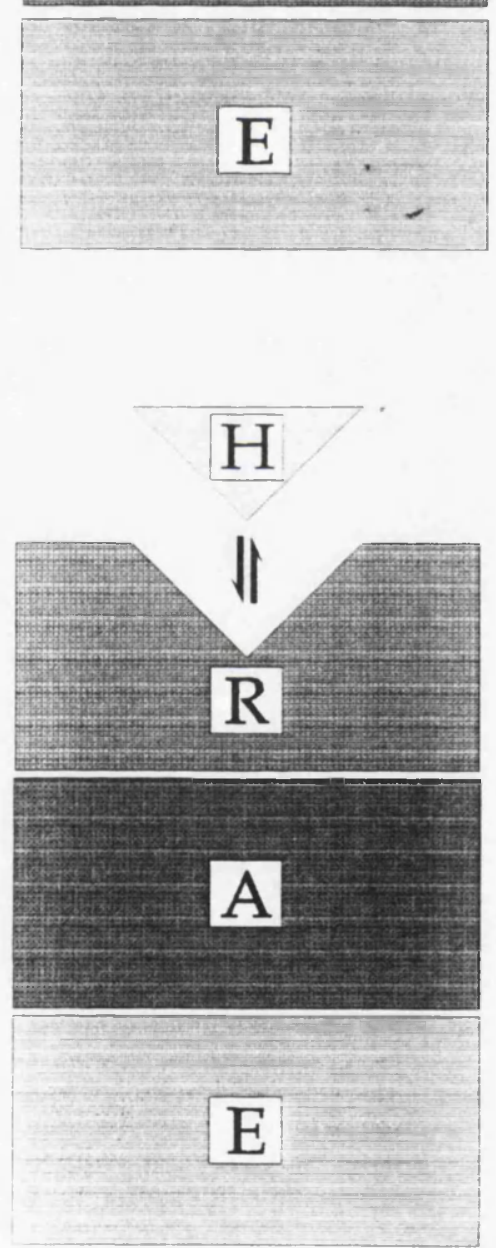

Mechanism 3

Figure 2.3 Proposed Mechanisms for the Action of Hormones 
which acts as a transcriptional regulator. This regulatory effect is achieved by altering the transcription rates of steroid responsive genes or, occasionally, the post transcription process affecting a change in the levels of specific mRNA and/or proteins $^{18}$.

Unlike some other hormones that exert a very rapid response, e.g. adrenaline, the time period over which steroid action occurs is quite long. It takes around four minutes for the steroid to diffuse to the nucleus and bind to the receptor protein and 4-5 minutes for the steroid-receptor complex to become attached to the steroid response element. Once bound to the SRE, it can be up to four hours for the Pre-mRNA to be formed, up to 4 hours for the mRNA to be transcripted and 4-8 hours for the levels of enzymes and proteins to be affected, either stimulating or diminishing production levels. The major physiological effects of steroids on an individual are not observed until 12-24 hours after dosing.

\section{Structure of a Steroid Receptor}

In-depth studies have been carried out on progesterone receptors in chickens ${ }^{19}, 20$ and rabbits ${ }^{21}$, glucocorticoid receptors in mouse ${ }^{22}$, human ${ }^{23}$, and rat ${ }^{24}$, and oestrogen receptors in chicken ${ }^{25}$, frog $^{26}$, human ${ }^{27}$, mouse ${ }^{28}$ and rainbow trout ${ }^{29}$. As a result of these studies, it has been proposed that there are four distinct regions or domains in each receptor (Figure 2.5). The N-terminal region (Domain 1) of the receptor is primarily concerned with the activation of transcription, Domain 2 is the DNA-binding domain and contains two 'zinc fingers' which wrap around the DNA helix, Domain 3 is termed the hinge domain and is involved in nuclear localisation, transcription and dimer formation and domain 4 , the steroid binding domain is where the steroid actually binds to the receptor. When the steroid binds with the receptor, a conformational change occurs to the receptor and this allows the central region of the receptor to bind to the DNA and allow the hormone to exert its effects. 


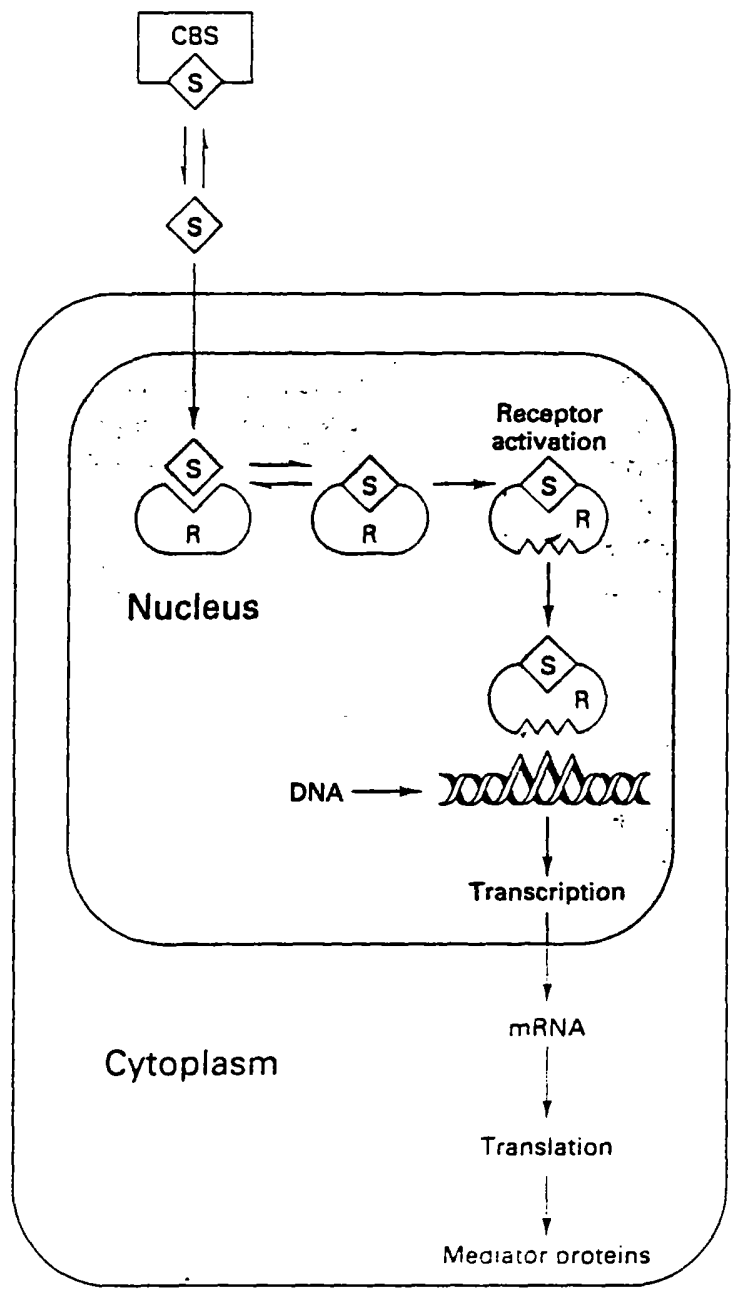

Figure 2.4 Mechanism of Steroid Action. 


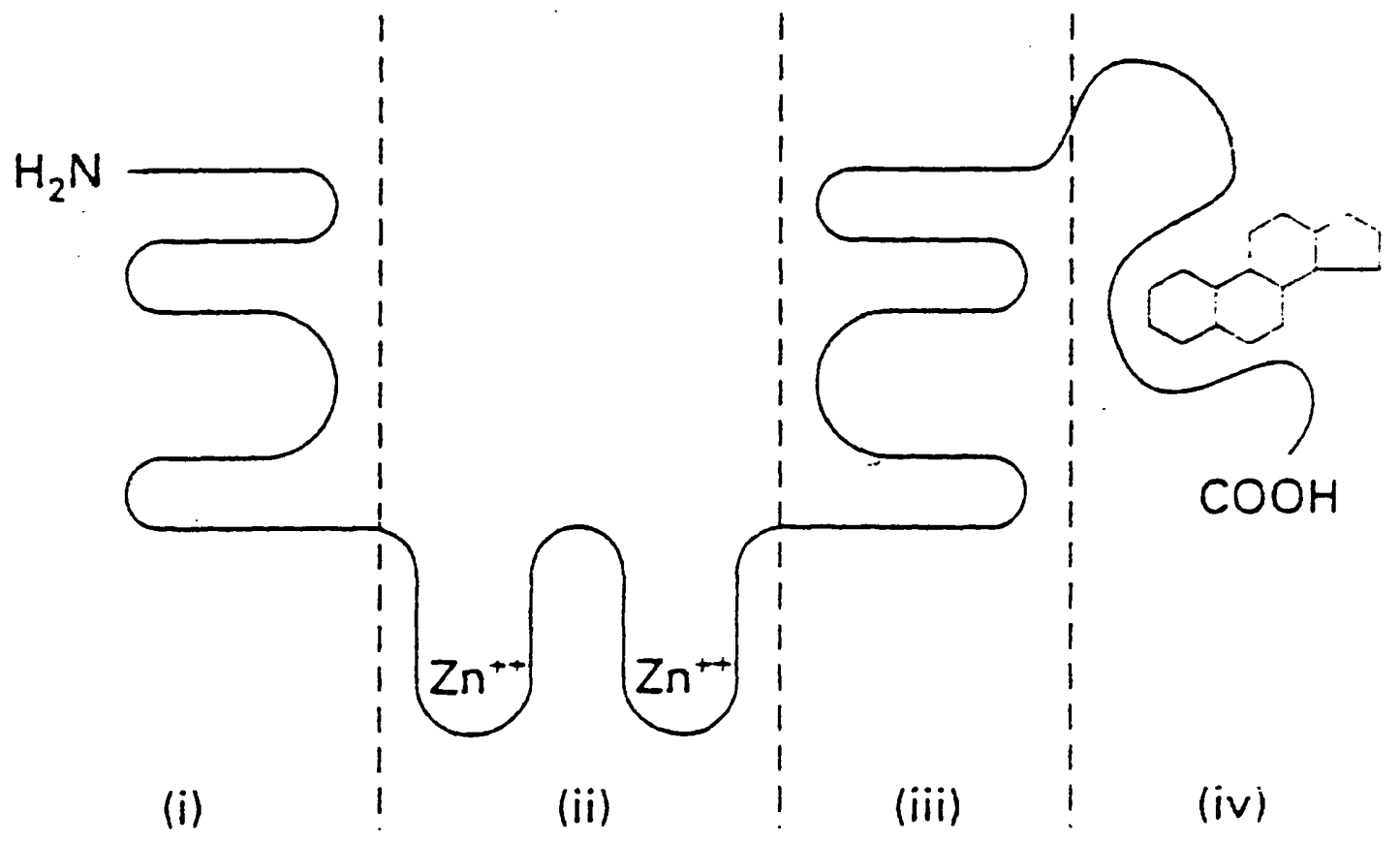

Figure 2.5 Structure of Steroid Receptor 


\section{Biosynthesis of Steroids.}

In vivo, the mammalian synthesis of steroids occurs via cholesterol. It has become accepted that a small but quickly replenished pool of cholesterol exists which does not come from circulating cholesterol but which is derived mainly from synthesis from acetyl units. Figure 2.6 summarises the synthetic processes.

Three molecules of acetyl CoA combine to form mevalonic acid, a six carbon molecule (C6). This is decarboxylated and dehydrogenated to form isopentylpyrophospate, the basic $\mathrm{C} 5$ isoprenoid building block. Three of these $\mathrm{C} 5$ units combine to give farnesyl pyrophosphate (C15) and two of these farnesyl units join to give squalene (C30). Squalene is cyclised to lanosterol, which is subsequently modified by demethylation, reduction and double bond re-arrangement to cholesterol.

\section{Conversion of Cholesterol to Pregnenolone}

$3 \beta$-Hydroxy- $\Delta 5$-pregnan-20-one (pregnenolone) is now considered to be the precursor of $\mathrm{C} 21, \mathrm{C} 19$, and $\mathrm{C} 18$ steroids. It is thought that cholesterol undergoes a two-stage process of conversion to 20,22-dihydroxycholesterol followed by side chain cleavage to give pregnenolone and a C6 unit, 4-methylpentanal, which is rapidly converted to isocaproic acid (Figure 2.7). There is uncertainty as to which of the two hydroxylations take place first. Experimental evidence from the late 1950's suggested that $\mathrm{C} 20$ was the first position to be hydroxylated followed by hydroxylation at C22. During the past twenty years however, several hydroxy- and epoxy- derivatives of cholesterol of known stereochemistry have been synthesised and Hume et al. have provided evidence for the initial hydroxylation to take place on $\mathrm{C} 22^{30}$. Further studies on this system have shown the presence of three enzymes, two hydroxylases and one lysase with a requirement for molecular oxygen and NADPH in association with two proteins, adrenodoxin and adrenodoxin reductase. Simpson provides a detailed review of this work ${ }^{31}$. 
<smiles>CC(=O)SC(C)=O</smiles><smiles>[3H][3H]</smiles><smiles>CC(O)(CCO)CC(=O)O</smiles><smiles>[3H]C</smiles><smiles>C=C(C)CCOP(=O)(O)O</smiles><smiles>[3H][3H]</smiles><smiles>CC(C)=CCCC(C)=CCCC(C)=CCOP(=O)(O)O[PH2+]</smiles><smiles>CC(C)=CCC/C(C)=C/CC/C=C(\C)CC/C=C(\C)CCC=C(C)C</smiles><smiles>C[14CH2]C(C)C1CCC2(C)C3=C4CCC5C(C)(C)C(O)CCC5(C)C4(C)CCC3(C)C12CCC=C(C)C</smiles>

Figure 2.6 Synthesis of Cholesterol from Acetyl CoA. 


\section{The Biosynthesis of Androgens}

The synthesis of androgens can occur in the testes, adrenal cortex, ovaries and the placenta with the largest portion of biosynthesis occurring in the Leydig Cells in the testes of males. The synthesis of androgens has been extensively studied in many species including humans and dogs. There are two possible closely related pathways for the synthesis of testosterone in the body; the $\Delta 5$ pathway, involving $3 \beta$ hydroxy- $\Delta 5$ steroids, which appears to be the favoured path in human and dog biosynthesis, and the $\Delta 4$ pathway, favoured in rat and mouse, involving 3-oxo-4-enesteroids (Figure 2.7).

\section{$\Delta 5$ Pathway.}

Pregnenolone is converted to $17 \alpha$-hydroxypregnenolone and is followed by side chain cleavage to give dehydroepiandrosterone (DHA). Both these steps have a requirement for NADPH and oxygen. DHA is converted to androstenediol and the final stage involves the re-arrangement of the $\Delta 5$ double bond to $\Delta 4$ and the dehydrogenation of the hydroxy group at position 3 to give testosterone.

\section{$\Delta 4$ Pathway}

Except for the initial stage, the $\Delta 4$ pathway follows a similar pattern to the $\Delta$ 5 pathway. Pregnenolone is transferred to progesterone by the conversion of the saturated A-ring to a 4-ene-3-one structure. This is hydroxylated at position 17 to give $17 \alpha$-hydroxyprogesterone. Following side chain cleavage to give 4-androsten3,17-dione, the final stage of the synthesis is the reduction of the ketone group at position 17 to give testosterone. As in the $\Delta 5$ pathway, the two stage conversion of progesterone to 4-androsten-3,17-dione has a requirement for NADPH and oxygen.

There exists the possibility for the interconversion of $3 \beta$-hydroxy-5-ene steroids to 3-oxo-4-ene steroids, i.e. the $\Delta 5$ to the $\Delta 4$ pathway. It was initially thought that there were two separate enzymes required to accomplish this conversion; a $3 \beta$-hydroxy NAD oxidoreductase and a 3-oxosteroid-4,5-isomerase. It has more recently been suggested that these enzymes form part of a complex ${ }^{32}, 33$. 

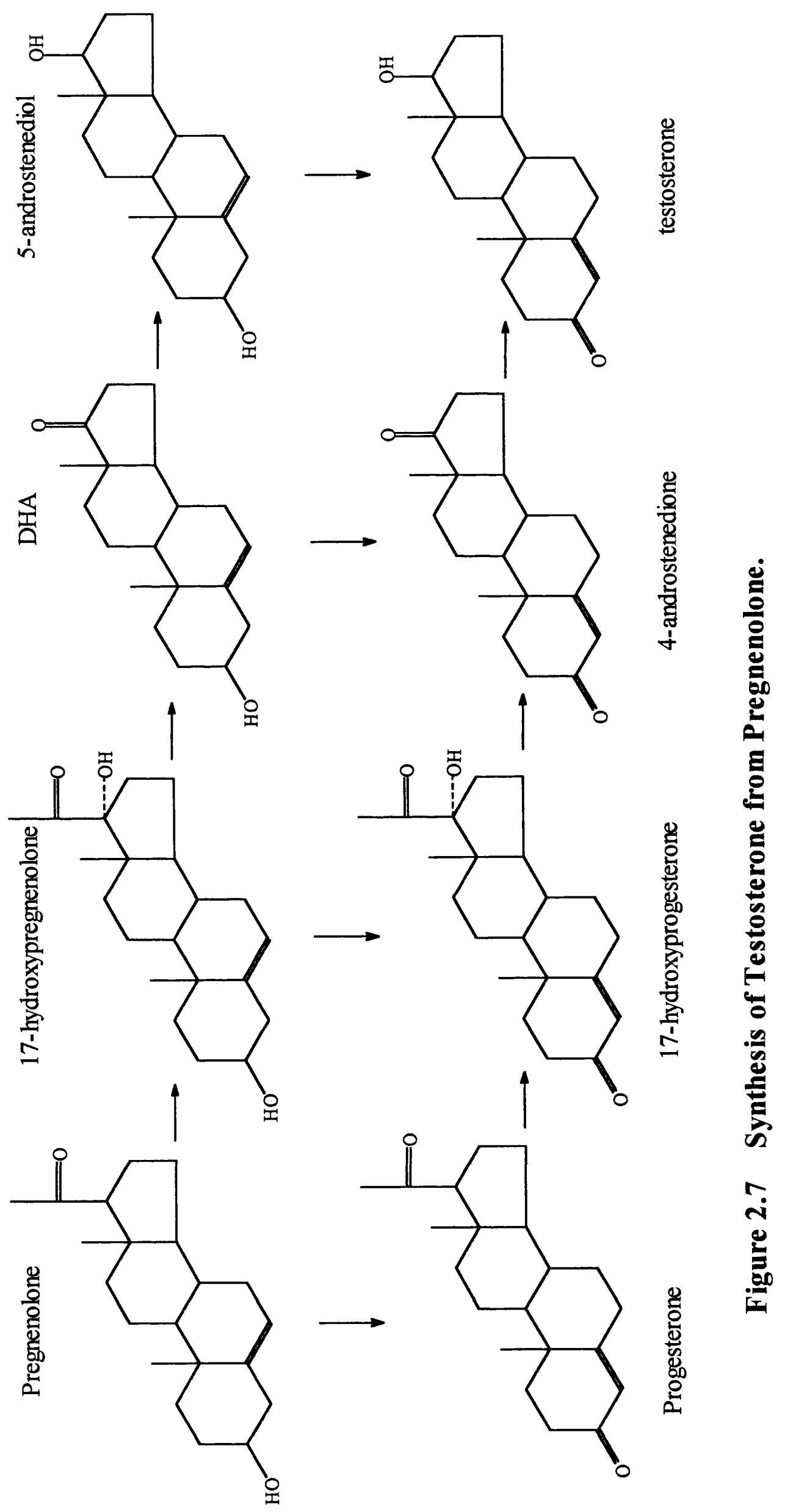


\section{The Biosynthesis of Corticosteroids}

Using radiolabelled precursors, the basic pathways for the synthesis of corticosteroids have been established. The initial stage appears to be the conversion of pregnenolone to progesterone. Once progesterone has been formed the synthetic pathway can take one of two routes. If progesterone is hydroxylated at C21, deoxycorticosterone (DOC) is formed. This can be followed by hydroxylation at C11 to give corticosterone. Corticosterone can be converted to aldosterone, the major mineralocorticoid by an 18-hydroxysteroid dehydrogenase.

If progesterone is hydroxylated at $\mathrm{C} 17$ to give 17-hydroxyprogesterone, this leads to a further branching of the system. The 17-hydroxyprogesterone can be diverted into androgen synthesis (see above) or can be hydroxylated again at C21 to give 11-deoxycortisol before a further hydroxylation to give cortisol (Fig 2.8). All the enzymes in this system have a requirement for NADPH and oxygen.

\section{The Biosynthesis of Oestrogens}

In humans, there are four main organs involved in the production of oestrogens. These are the ovaries ${ }^{34}$, the testes ${ }^{35}$, the adrenal cortex and the foetoplacental unit. The main oestrogen synthesised is oestradiol along with significant amounts of oestrone and oestriol. The initial stages in oestrogen synthesis are identical to the $\Delta 4$ and $\Delta 5$ pathways described above and illustrated in Figure 2.7.

The conversion of 4-androstenedione and testosterone to $17 \beta$-oestradiol follows similar paths. The methyl group on $\mathrm{C} 19$ is oxidised and is followed by conversion to a phenolic compound (Figure 2.9). The removal of the carbon at C19 appears to be achieved by the hydroxylation of the A-ring at C2 followed by nonenzymatic aromatisation ${ }^{36,37}$. As with the synthesis of androgens and corticosteroids, there is a requirement for NADPH and oxygen. Although attempts have been made to characterise the aromatase system, the results have not yet been successful ${ }^{38,39}$. 


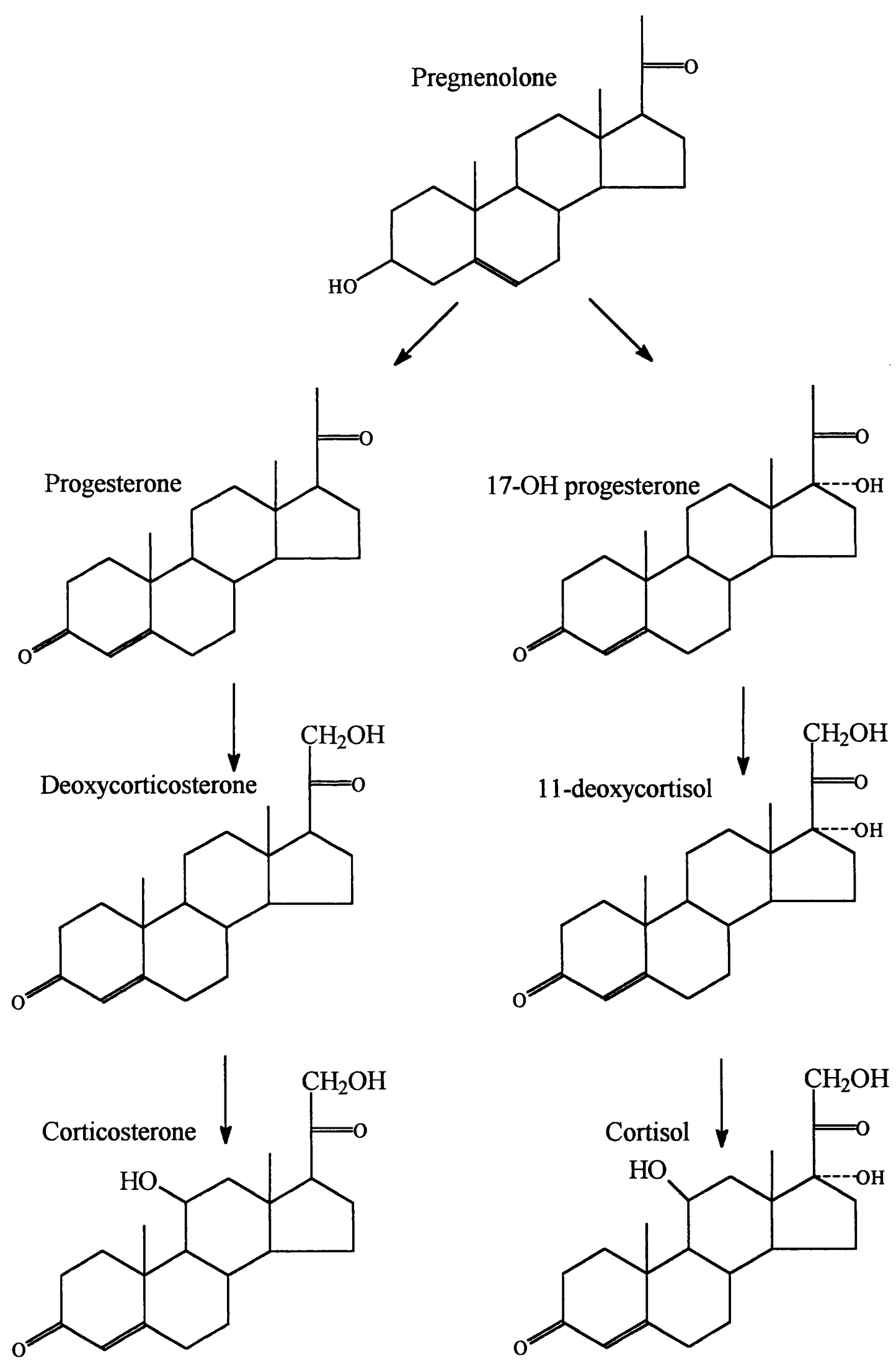

Figure 2.8 Biosynthesis of Corticosteroids 


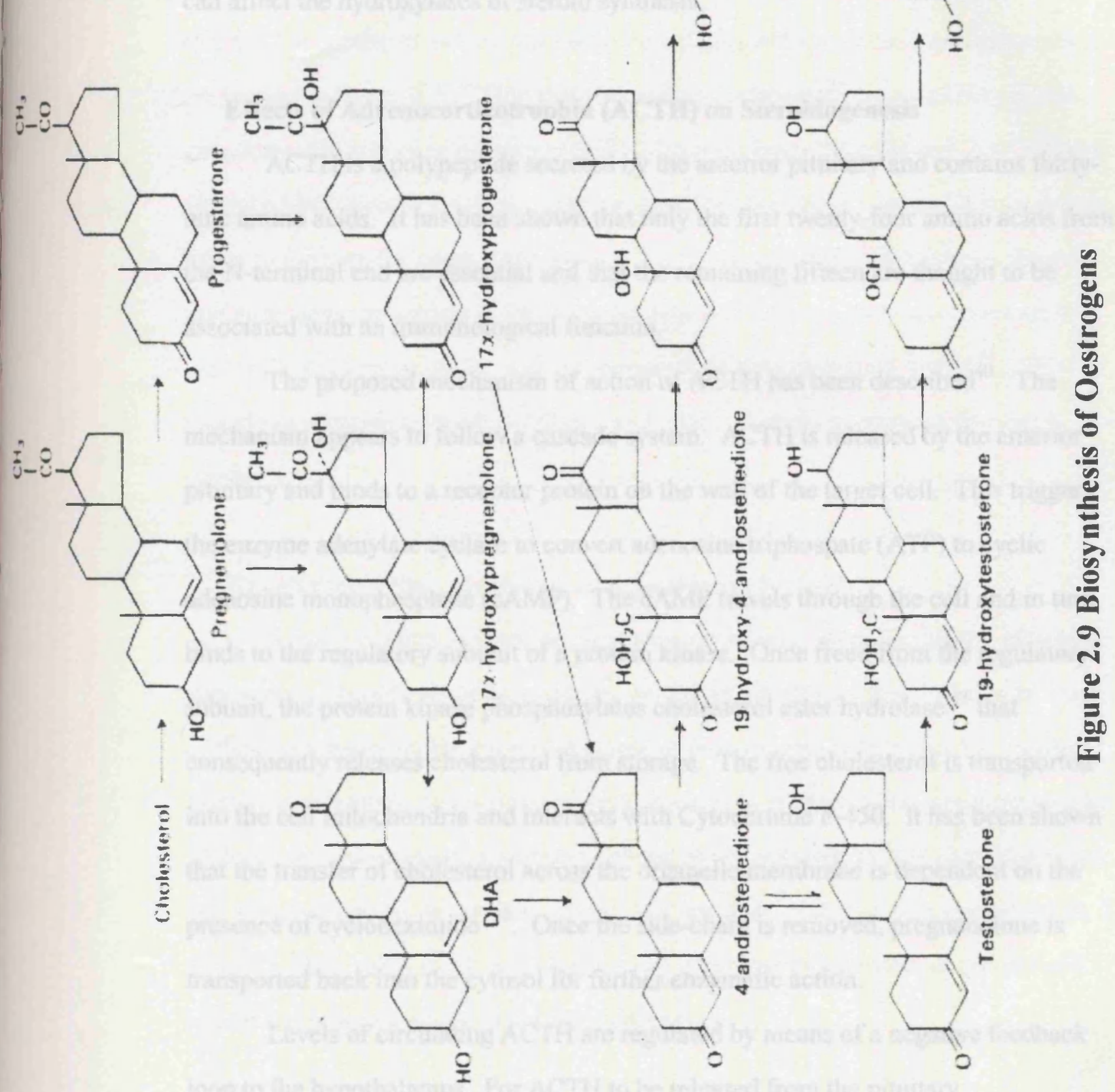




\section{$\underline{\text { Regulation of Steroidogenesis }}$}

There appear to be many factors affecting the regulation of steroidogenesis. The trophic hormones, e.g. adrenocorticotrophin (ACTH), exhibit regulatory effects and it has been shown that the presence or absence of various endogenous steroids can affect the hydroxylases of steroid synthesis.

\section{Effects of Adrenocorticotrophin (ACTH) on Steroidogenesis}

ACTH is a polypeptide secreted by the anterior pituitary and contains thirtynine amino acids. It has been shown that only the first twenty-four amino acids from the $\mathrm{N}$-terminal end are essential and that the remaining fifteen are thought to be associated with an immunological function.

The proposed mechanism of action of ACTH has been described ${ }^{40}$. The mechanism appears to follow a cascade system. ACTH is released by the anterior pituitary and binds to a receptor protein on the wall of the target cell. This triggers the enzyme adenylate cyclase to convert adenosine triphospate (ATP) to cyclic adenosine monophosphate (cAMP). The cAMP travels through the cell and in turn binds to the regulatory subunit of a protein kinase. Once freed from the regulatory subunit, the protein kinase phosphorylates cholesterol ester hydrolase ${ }^{41}$ that consequently releases cholesterol from storage. The free cholesterol is transported into the cell mitochondria and interacts with Cytochrome P-450. It has been shown that the transfer of cholesterol across the organelle membrane is dependent on the presence of cycloheximide ${ }^{42}$. Once the side-chain is removed, pregnenolone is transported back into the cytosol for further enzymatic action.

Levels of circulating ACTH are regulated by means of a negative feedback loop to the hypothalamus. For ACTH to be released from the pituitary, corticotrophin releasing factor (CRF), a forty-one amino acid peptide hormone, must be released by the hypothalamus ${ }^{43,44}$. The release of this factor is inhibited by the 
presence of circulating steroids and it has been shown that CRF only affects the release of ACTH and no other circulating hormone levels ${ }^{45}$.

\section{Regulatory Effects of Gonadotrophins on steroidogenesis}

In humans, there are two main protein hormones that are secreted by the pituitary. These are luteinising hormone $(\mathrm{LH})$ and follicle stimulating hormone (FSH). The modes of action, release and regulatory effects of these gonadotrophins are similar to those of ACTH in that their release is triggered by a smaller peptide, gonadotrophin releasing hormone $(\mathrm{GnRH})$ from the hypothalamus and their action is via the cAMP protein kinase mechanism described above. A negative feedback loop exists to the hypothalamus that regulates the release of GnRH. It has been shown that androgens participate in this negative feedback loop, as there are increases in the levels of secretion of the gonadotrophins following castration in rats ${ }^{46}$. LH is thought to regulate androgen synthesis and FSH is thought to regulate oestrogen synthesis.

\section{Regulation of Steroidogenesis by Endogenous Steroids}

A large volume of work has been concerned with investigating the effects of endogenous steroids on the biosynthetic pathways. It appears that the later products of these pathways inhibit the earlier stages of the synthesis (Fig 2.10). For example, testosterone inhibits the conversion of cholesterol to pregnenolone, pregnenolone to 17-hydroxypregnenolone, 4-androstenedione to testosterone, DOC to corticosterone and 11-deoxycortisol to cortisol. It does not however inhibit its own conversion to 5 $\alpha-\mathrm{DHT}$. This would result in a build up in the amount of testosterone present and inhibit the previous stages to a greater degree.

\section{Metabolism of Endogenous Steroids}

The purpose of metabolism is normally twofold; to reduce the physiological activity of a compound and to increase the ability of an organism to excrete the material from its system. In the case of steroids, the reduction in physiological 


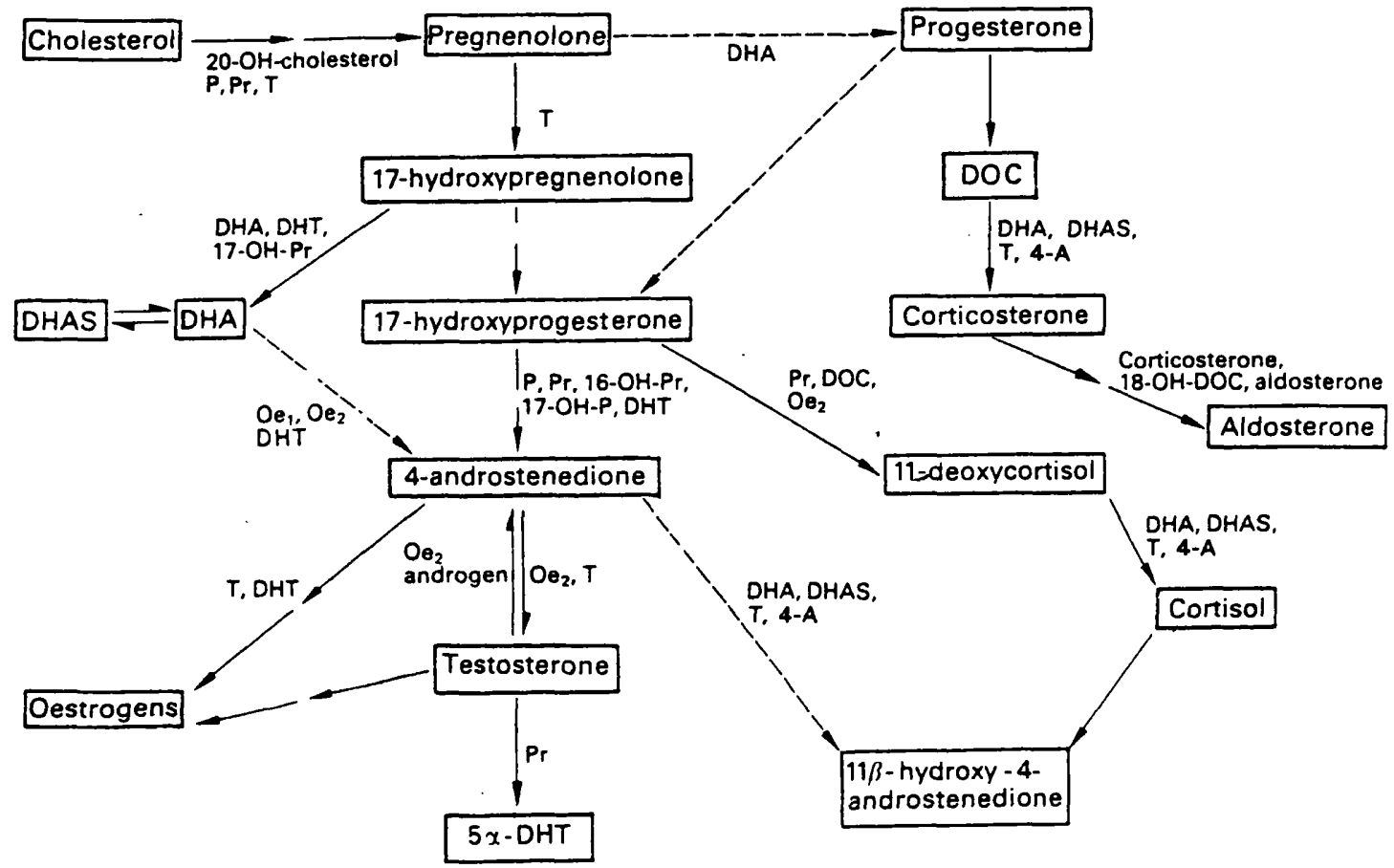

Figure 2.10 The Inhibition of Steroid Synthesis by Steroids 
activity goes hand in hand with an increase in hydrophilicity of the compounds. Four major catabolic routes have been established.

\section{The reduction of the $\mathrm{C} 4$ double bond and the accompanying reduction of}

\section{the C3 oxo group.}

This catabolic process can result in the formation of up to four metabolites, all stereoisomers. These are $5 \alpha-3 \beta, 5 \alpha-3 \alpha, 5 \beta-3 \beta$, and $5 \beta-3 \alpha$ where the stereochemistry of position 5 relates to the hydrogen atom and position 3 to the hydroxy group (See Figures 2.1 and 2.2 ). In man the $3 \alpha-5 \beta$ hydroxysteroids predominate but in many animals the $3 \beta$-hydroxysteroids are to the fore. It appears as if this catabolic step is a two-stage process. The initial stage involves the reduction of the double bond by either a 4 -en- 5 - $\alpha$-reductase or a 4-en-5- $\beta$-reductase and is followed by either a $3 \alpha$ - or a $3 \beta$-hydroxysteroid dehydrogenase.

\section{2) The reduction of the C20 oxo group to a secondary alcohol.}

In a similar route to the previous catabolic pathway, the C20 oxo group is reduced by a $20 \alpha$ - or a $20 \beta$-hydroxysteroid dehydrogenase. It is thought that the $20 \alpha$ form predominates slightly over the $20 \beta$ isomer in man.

\section{3) The oxidation of the 17-hydroxy group.}

The 17-hydroxy group is converted to the 17-oxo group by either a $17 \alpha$ - or a $17 \beta$ - hydroxysteroid dehydrogenase.

\section{4) Hydroxylation throughout the ring system.}

It is possible for some corticosteroids, for example cortisol, to become hydroxylated on $\mathrm{C} 6$ or C16. These hydroxylations form quite polar molecules and allow the compound to be excreted from the system in an unconjugated form (see urinary excretion below). 


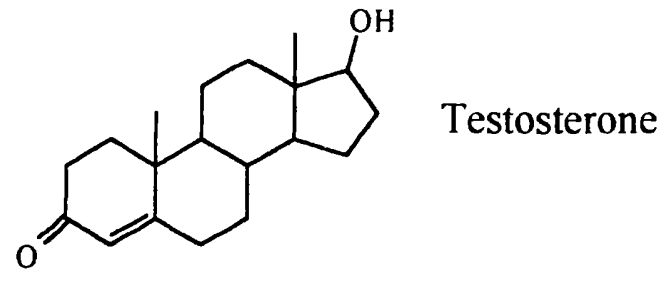

4<smiles>CC12CCC3C(CCC4=CC(=O)CCC43C)C1CCC2=O</smiles>

\section{4-androstenedione}

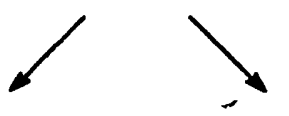

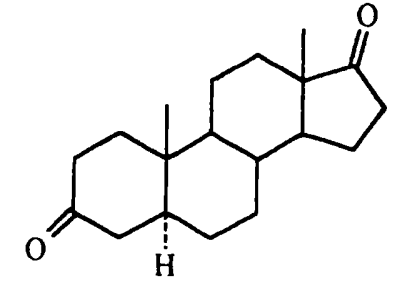

$5 \alpha$-androstane-3,17-dione

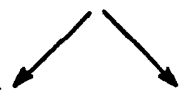

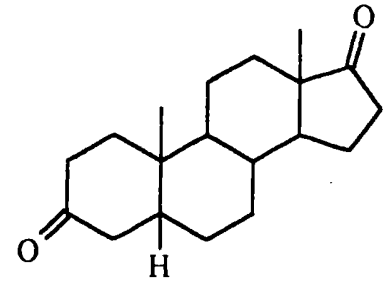

$5 \beta$-androstane-3,17-dione

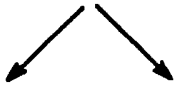

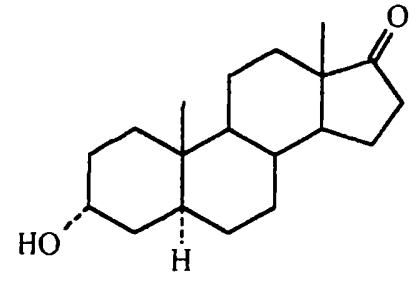

Androsterone

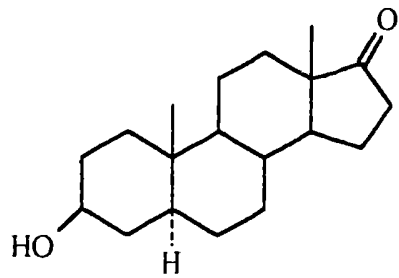

Epi-androsterone

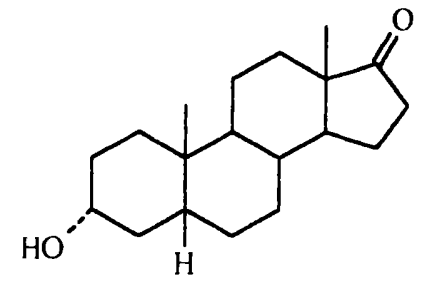

Aetiocholanolone

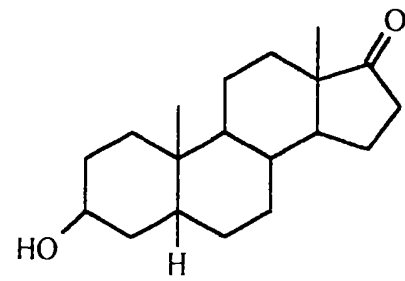

Epi-aetiocholanolone

Figure 2.11 Metabolism of Testosterone 
The metabolism of testosterone is summarised in Figure 2.11 and shows the combination of two of the catabolic processes described above. Testosterone is initially oxidised at the hydroxy group on $\mathrm{C} 17$ to give 4-androstenedione. This then undergoes the first catabolic process described and can give rise to up to four metabolites; androsterone, epiandrosterone, etiocholanolone and epietiocholanolone.

\section{Excretion of Steroids}

There are two major routes of excretion. These are urinary and biliary and have been the pathways most examined. Excretion of steroids has also been examined in other body fluids, e.g. saliva, but levels of excreted compounds have been found to be low.

\section{1) Urinary Excretion}

Almost all steroids, except the polyhydroxylated metabolites, are too hydrophobic to be excreted directly. To overcome this problem, the steroids are converted to glucuronide or sulphate conjugates.

\section{Glucuronidation}

Glucuronidation involves the addition of a molecule of glucuronic acid to functional groups within the target molecule. In steroids, this target group is normally a hydroxyl group.

Glucose-1-phosphate is combined with uridine triphosphate (UTP), employing the enzyme UDP-glucose pyrophosphorylase, to give uridine diphosphate glucose (UDP-glucose). The UDP-glucose molecule is then dehydrated using UDPglucose dehydrogenase and $\mathrm{NAD}^{+}$to give UDP-glucuronic acid. This compound then combines with a functional group on the target molecule, using UDPglucuronysl transferase, to give UDP and the glucuronide conjugate. Work has 
shown that the 17-oxo steroids are primarily excreted as the glucuronide conjugate with less than $10 \%$ excreted by other routes, mainly sulphates 47,48 .

\section{Sulphation}

Initially, the conversion of steroids to sulphates was thought only to be a mechanism to allow the conjugates to be excreted via the kidneys. The use of radiolabelled steroids and the examination of DHA sulphate concentrations in arterial blood going into the adrenals and venous blood leaving the adrenals showed that these organs were synthesising the conjugate ${ }^{49}$.

The sulphate group reacts initially with ATP to produce APS, adenosine-5'phosphosulphate and pyrophosphate. The APS combines with a second molecule of ATP under the influence of ATP kinase to give PAPS, 3'-phosphoadenosine-5'phosphosulphate and ADP. Finally PAPS combines with the hydroxyl group on the steroid to give the sulphate, PAP, 3',5'-phosphoadenosine and a proton.

The purpose of steroid sulphates in the blood stream is subject to speculation. They may act as a circulating store of steroid precursors ${ }^{50}$ because the sulphates are not removed so quickly from the blood supply as free steroids.

\section{Biliary Excretion.}

Although the principal route of excretion is urinary, a significant amount of steroids can be excreted via the bile. Sandberg and Slaunwhite ${ }^{51}$ gave human volunteers radiolabelled testosterone and recovered $89 \%$ of the dose in the urine and $6 \%$ of the dose in the faeces. These steroids tend to be excreted as conjugates and it has been suggested that they can be classified into three groups, depending on the length of time it takes for them to be eliminated from the body ${ }^{52}$. Group III steroids include cortisol and members of this group tend not to be eliminated to any significant degree in the bile. Group II steroids include testosterone and, as stated above, are excreted in the bile. Group 1 steroids include oestrone and oestradiol and it may take up to five days for radiolabelled metabolites to be recovered from 
the urine and the faeces ${ }^{53}$. This prolonged period of elimination has been attributed to enterohepatic recirculation that occurs when a compound is excreted via bile. The bile passes into the gastrointestinal tract and the compounds are re-absorbed from the small intestine and enter the circulation of the organism for a second time, thus giving a second "dose" of the material. The majority of the steroids found in bile are conjugated and unsaturated while almost all the steroids found in faeces are free and saturated. It has been suggested that intestinal bacteria, probably in the caecum, metabolise the conjugated biliary steroids and it is the free steroids that are re-absorbed. The re-absorbed material can then be eliminated from the body a second time and may follow either primary route of excretion, and consequently be subject to further episodes of recirculation.

\section{Anabolic Androgenic Steroids (AAS)}

The principal androgenic steroid in the male is testosterone and in humans, a healthy young male will synthesise approximately 7 milligrams per day. During puberty, androgens control the growth of the primary sex organs in the male. All androgens also have an anabolic effect. This anabolic effect promotes an increase in muscle protein, accompanied by an increase in urinary creatinine, decrease in urinary nitrogen but no increase in blood urea. These result in a positive nitrogen balance, caused by an increase in metabolic nitrogen pool. Changes in bone structure and levels of enzymes associated with protein metabolism also occur and this gives rise to the characteristic growth during puberty. Clinically, the androgenic properties of certain steroids are used in replacement therapy in males suffering from hypogonadal disorders, in adolescent males with delayed puberty or growth, and in females androgens can be useful in the management of breast carcinomas. The anabolic properties of steroids are used to build up muscle bulk and cause a positive nitrogen balance in individuals who may be in a weakened state. 
It is not possible to have an androgenic steroid that does not have an anabolic effect and vice versa. It is however possible to synthesis a steroid with a high anabolic/androgenic ratio i.e. maximise the anabolic effects while minimising the androgenic effects. This ratio can be assessed by determining the relative changes in the weight of the levator anti muscle and the seminal vesicles in 21 day old castrated rate and expressing these changes as a ratio. The higher the ratio, the higher is the anabolic/androgenic ratio.

It is for the anabolic effect that steroids are primarily used in sport, in order to increase muscle bulk and strength, and to promote tissue repair and recovery following damage caused by intensive training or competition. It has been reported that individuals will take doses far in excess of the normal therapeutic amounts to achieve the desired anabolic effect. Testosterone is the primary androgenic/anabolic steroid produced by males. If testosterone is taken orally it is subject to extensive first pass metabolism and consequently can cause little effect in the body. Testosterone exhibits a plasma half-life of between 10 and 21 minutes in humans in the blood. It is metabolised to androstendione that exhibits only weak androgenic/anabolic properties. If unmodified testosterone is given parenterally, it is absorbed from the injection site and again is rapidly metabolised. Chemical modification of the structure of the androgen nucleus overcomes these problems and can produce anabolic steroids which are far more effective both as anabolic agents (i.e. high anabolic/androgenic ratio) and which have a far longer action. There are three general ways in which the nucleus can be altered to produce these effects:

1. esterification of the $17 \beta$ hydroxyl group

2. alkylation of the $17 \alpha$ position

3. modification of the ring structure

Table 2.1 lists some of the synthetic anabolic steroids commercially available. It is possible to use only one of the above chemical modifications or all 


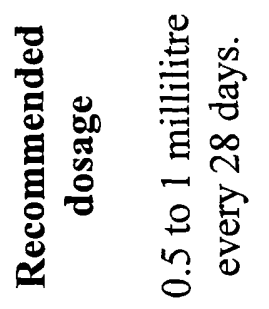

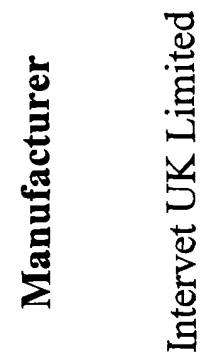

亳

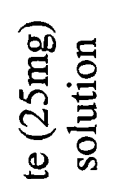

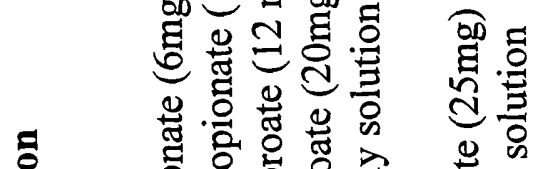

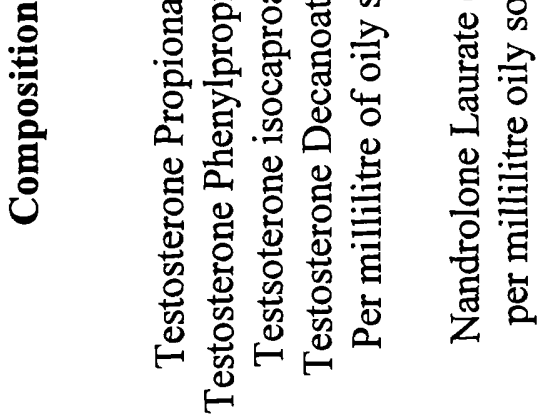

苟
0
0
0
0
0
0
0
0
0

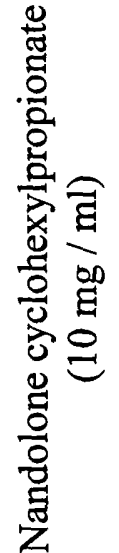

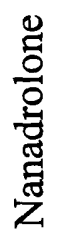

童
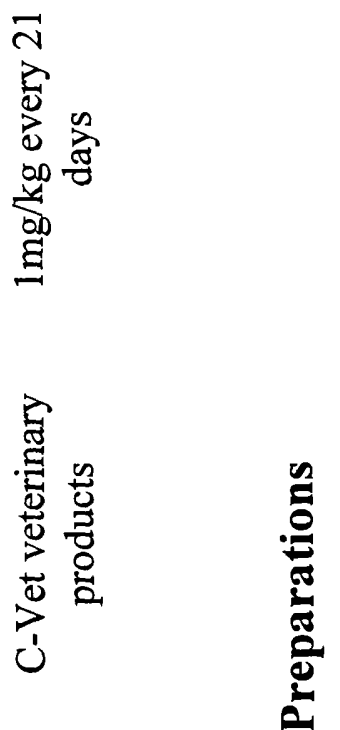

응

送

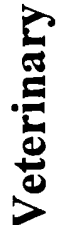

릴

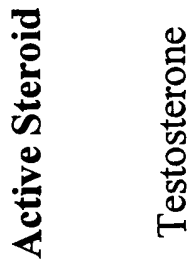

$\frac{0}{0}$
$\frac{0}{0}$
$\frac{0}{0}$
Z

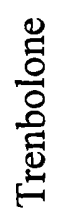

ڤึ

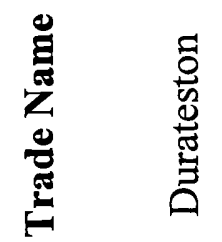

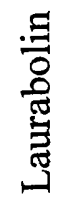

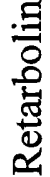


three to create an effective anabolic agent. The $17 \alpha$ alkylated steroids are effective orally. They are only slowly metabolised by the liver and consequently are not subject to extensive first pass metabolism. These oral steroids include methyltestosterone (Android) stanozalol (Winstrol) and oxandrolone (Anavar). For effective parenteral administration, esters of the anabolic agents are used. The esters are suspended in an oily base and, once injected, are slowly absorbed from the depot, and hydrolysed to give the free steroid. In humans, an injection of $200 \mathrm{mg}$ of testosterone enanthate will give peak serum levels between 1 and three days after injection with levels falling to normal during the next 10-41 days. Other routes of administration are available (transdermal patches and long term implants ${ }^{54}$ ) but these are less liable to be used covertly.

\section{Metabolism of Exogenous Anabolic Steroids.}

A large body of information exists regarding the metabolism of anabolic agents in mammals. Table 2.2 summarises the principal metabolites of some of the more common anabolic/androgenic steroids in various species. The metabolism of steroids by the racing greyhound will be covered in detail later in this chapter.

Major advances in technology have allowed a more detailed elucidation of the metabolic patterns of steroids. Houghton et al in 1978 identified two metabolites of 19-nortestosterone in horse urine following the administration of nandrolone and nandrolone esters, using packed column gas chromatography mass spectrometry ${ }^{61}$. Later studies by Masse et al. ${ }^{63}$ and Schanzer et al. ${ }^{64}$ utilised capillary gas chromatography-mass spectrometry and identified 12 and 11 metabolites of stanozolol respectively. Both groups of authors concluded that almost all of these metabolites were hydroxylated and that the great majority was excreted as conjugates. Muck and Henion used liquid chromatography-mass spectrometry (LCMS) to examine the metabolism of stanozolol in humans and horses ${ }^{65}$. Six metabolites were identified in human urine but only four in equine urine. As with the previous studies, all the metabolites were hydroxylated. 
Inter species variations do exist and can be quite marked. The principal metabolite of trenbolone ( $17 \beta$-hydroxy trenbolone) in bovine urine is $17 \alpha$-hydroxytrenbolone. In the rat, however, the principal metabolites are trenbolone itself $(17 \beta-$ hydroxy trenbolone), 16-hydroxy-trenbolone and a 17-keto metabolite ${ }^{66}$. Care must therefore be exercised when extrapolating between species. Individuals who use anabolic agents for doping in sport also use quantities of steroids far in excess of normal therapeutic doses. This again may disrupt the excretion patterns of exogenous steroids. It is not ethically possible to repeat under controlled circumstances the use of such large doses of anabolic steroids. 


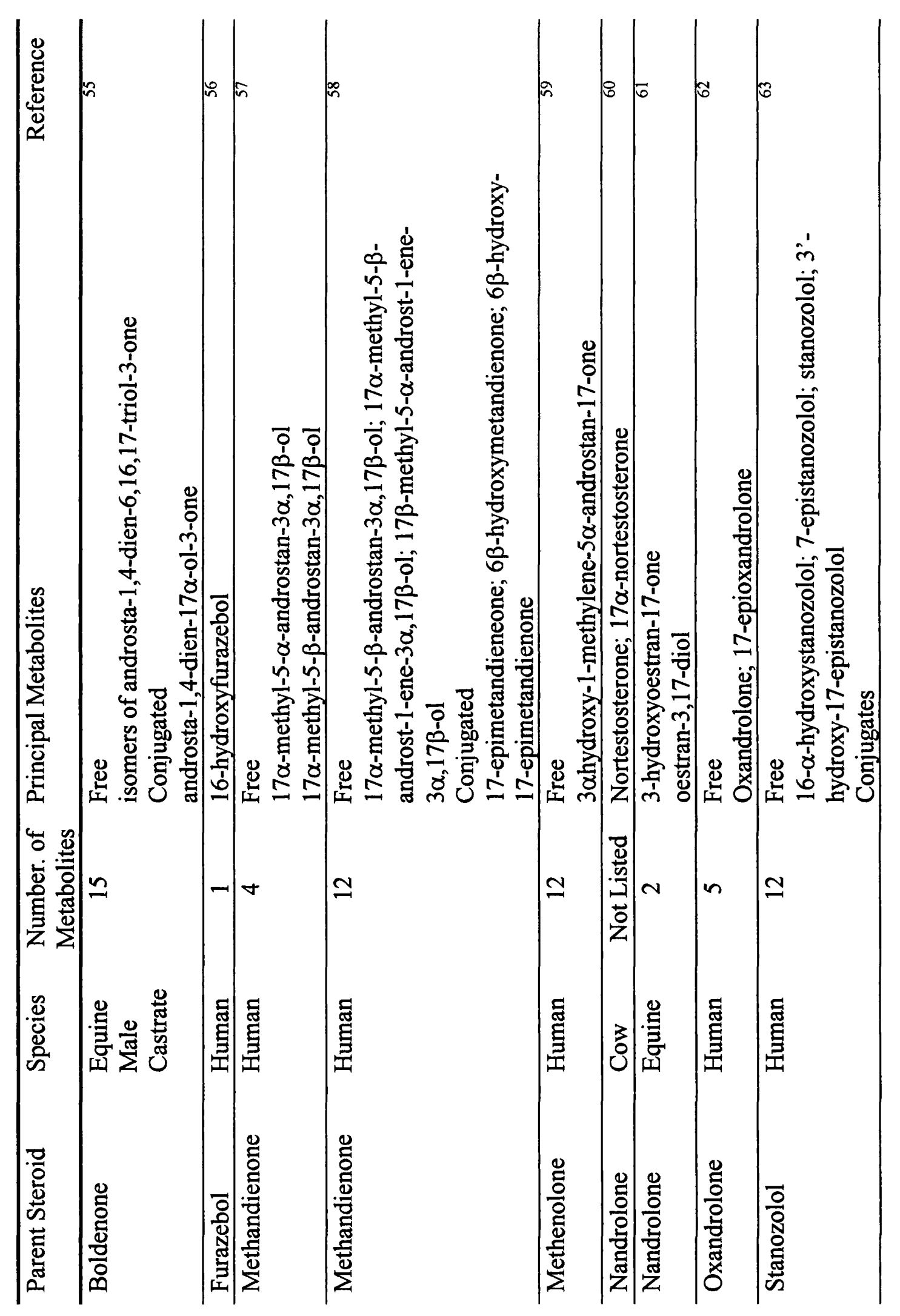




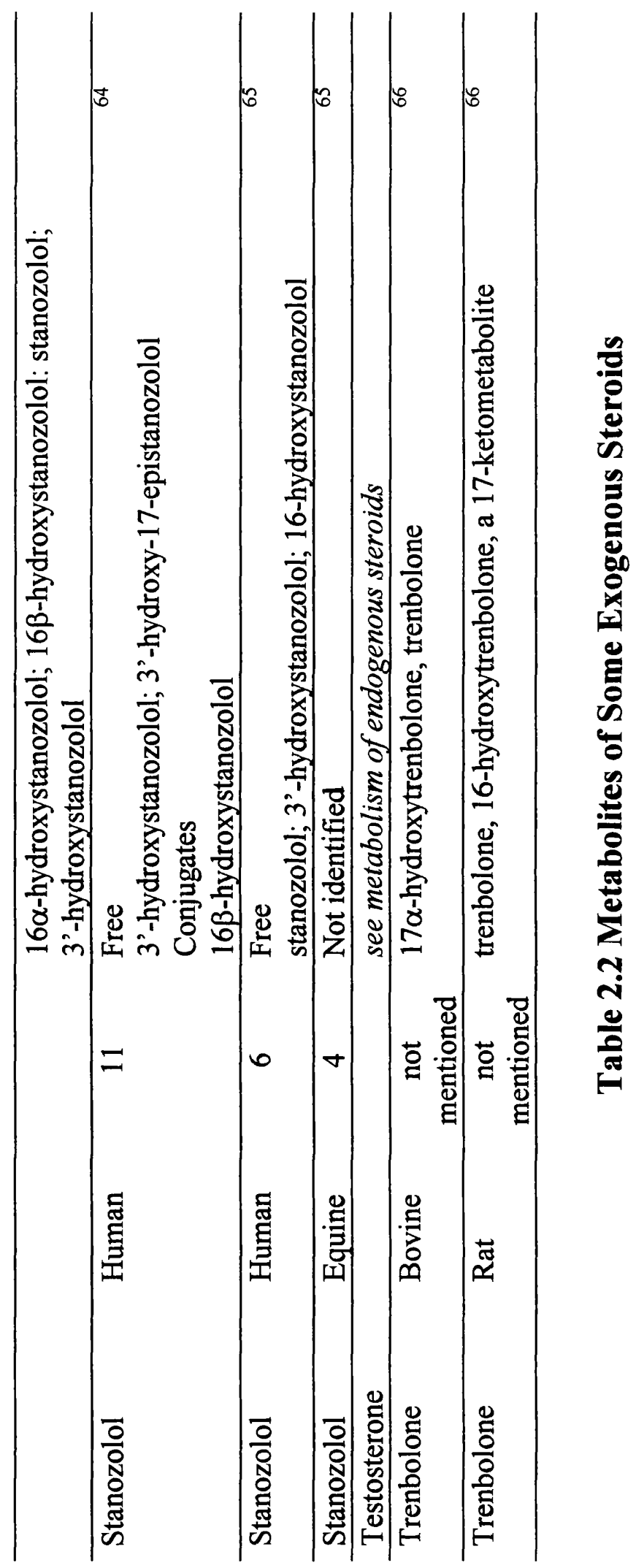


Schanzer and Donike have published details of the metabolism of a large number of synthetic steroids in $\operatorname{man}^{67}$. They detail the principal metabolites of 20 steroids, possible routes of preparation of authentic reference material and chromatographic data for the parent compounds and principal metabolites.

\section{Side Effects of Anabolic/Androgenic Steroids}

As indicated earlier, it is not possible to develop a purely anabolic steroid. Karch, in his book 'Pathology of Drug Abuse ${ }^{68}$, provides a review of the undesirable and unwanted side effects of anabolic steroid abuse in humans and some of these side effects are listed in Table 2.3. He divides the side effects and changes into four categories: social, physical, mental and clinical. Many of the listed changes apply to other species, and while it is possible that horses and greyhounds suffer social and mental changes, these effects can be difficult to assess.

Some of the physical changes listed can be attributed to the normal androgenic/anabolic activity of the steroids. The dangers associated with $17 \alpha-$ alkylated steroids concerning their ability to cause severe liver damage have been known for a long time ${ }^{69}$. The clinical changes are liable to be caused by the interference in the normal regulatory feedback systems for endogenous steroids. The mechanisms by which the mental changes occur have not yet been established. Some of these mental changes (increased aggression and manic episodes) have been grouped together and individuals who use anabolic steroids have suffered from a phenomenon known as 'roid rages'. Individuals who suffer from these 'roid rages' are reputed to be prone to violent outbursts of temper and/or aggression resulting in serious injury or death to others. The defence of 'roid rage' has been used in the USA, and in Scotland, individuals have tried to defend themselves from a charge of homicide by stating that their assailant was suffering a 'roid rage' when they were attacked. In the latter case, the defendants were eventually found guilty of culpable 
homicide and sentenced to 8 years in prison, even though the metabolites of nandrolone were identified in the urine of the murdered individual.

\section{Greyhounds and Steroids}

Very little information exists on steroids and greyhounds. Richkind and Enqvist ${ }^{70}$ examined the levels of corticosteroids in beagles and greyhounds using competitive protein binding. From their examination of six greyhounds, they concluded that the average concentration of corticosteroids was $18 \mathrm{ng}$ per millilitre of plasma in both dogs and bitches. There were no significant differences between the sexes nor was there any significant diurnal variation.

In one of the few published papers on anabolic steroids and greyhounds, Pemberton ${ }^{71}$ looked at the effect of various compounds on greyhounds, including the anabolic steroid nandrolone. He states that $25 \mathrm{mg}$ of nandrolone phenylpropionate intramuscularly caused a weight gain of approximately 1-2 kilograms per 2 weeks in female dogs. This body weight increase ceased after 2-3 kilograms had been gained. Weight gain exhibited by male dogs was less significant and Pemberton suggested that a cycle of one month on, one month off, would be more effective for dogs. He also examined the heart weight $(\mathrm{g})$ to body weight $(\mathrm{kg})$ ratio (Hwt/Bwt) in one dog and one bitch. The specific animals were chosen as they did not seem able to win a race. Following treatment with anabolic steroids, the dog's Hwt/Bwt increased from 10.1 to 11.6 and the female's from 10.9 to 13.2 . In addition, both animals started to win races.

One of the noted side effects of anabolic steroids is that when they are administered to growing individuals, the epiphyses may close up and stunt the growth of an individual. Pemberton states that he has never come across a case where this has occurred in greyhounds, but he has seen some animals that appeared to grow far too large due to the anabolic effect. Various blood parameters were also examined, and the biochemical changes that occurred were similar to those in humans, e.g. increased cholesterol. 


\begin{tabular}{l|l}
\hline Social & Obsession about health \\
Large amount of time in gyms \\
Large amount of food supplements and food substances \\
High calorie intake \\
No abuse of other drugs (including alcohol.)
\end{tabular}

\section{Table 2.3 Side effects of anabolic/androgenic steroid use}


There were however significant side effects. Virilisation occurred in the bitches along with suppression of oestrus, changes in sexual behaviour (rejection of a mate), occasional lack of enthusiasm to chase (for some anabolic steroids), increased aggression and a tendency to fight while racing. In males, testicular atrophy (with a 6-12 month recovery period) and increased aggression occurred. The side effects are similar to those found in humans and perhaps greyhounds can suffer a 'roid rage' like humans.

All the results reported by Pemberton appeared to be subjective in nature. There is no mention in the paper of systematic study or the use of control animals. In his experiments with dosing the greyhounds, two animals were used. Unfortunately, no indication is given of the prevalence of the side effects mentioned and very little detail is given regarding the racing form of the greyhounds prior to treatment with anabolic steroids, which apparently improved their racing ability.

Some attempts have been made to examine systematically the metabolism and excretion of anabolic agents in the racing greyhound. Brockwell et al. ${ }^{72}$ attempted to determine the metabolism of certain anabolic agents (testosterone, 19nortestosterone, 1,2-dehydrotestosterone) in greyhounds. The authors state that few publications were available regarding the metabolism of steroids in dogs and those that were available were primarily concerned with testosterone metabolism and bile metabolites. The authors gave mixtures of unlabelled and deuterium-labelled testosterone, 19-nortestosterone and 1,2-dehydrotestosterone to a castrated dog, intramuscular injections of boldenone undecylenate to an intact dog, and 19nortestosterone and a mixture of testosterone esters to an intact bitch. Although the authors were able to identify some metabolites of testosterone and 19nortestosterone, they concluded that excretion of the dosed steroids in urine was low and that immunoassay for the detection of steroids in urine was of little use. No attempt was made to try and establish a normal urinary steroid profile.

A related paper by the same group of authors concerning the detection of 19nortestosterone in greyhound urine provided little information regarding greyhounds 
and was concerned mainly with the application of an immunoassay for the detection of 19-nortestosterone in urine samples of horses ${ }^{73}$.

\section{Beta 2 Agonists}

Beta agonists are now banned under IOC regulations because of their reputed anabolic properties. Table 2.4 lists some of the $\beta-2$ agonists currently available. An agonist is simply a substance that activates a receptor and beta 2 is the designation of the receptor that is activated. Beta 2 receptors belong to the adrenoceptors of which there are five types, alpha $1(\alpha-1)$, alpha $2(\alpha-2)$, beta $1(\beta-1)$, beta- $2(\beta-2)$ and beta 3 $(\beta-3)$. Attribution of a specific receptor to a specific class is dependent on that receptor's response to specific agonists.

Originally developed to aid in the treatment of asthma sufferers, the $\beta-2$ agonists are thought to have anabolic or re-partitioning properties. Their use in cattle was reported in 1984 and the first use of $\beta-2$ agonists in humans is attributed to British athletes in 1988. Although the mechanism of anabolic action has not yet been explained, the basic mechanism of action of the adrenoceptors has been established. $\alpha-1$ Agonists activate phospholipase $C$ and $\alpha-2$ agonists inhibit adenylate cyclase and the formation of cyclic adenosine monophosphate (cAMP). All $\beta$-agonists act by the same mechanism and activate adenylate cyclase to stimulate the production of cAMP. Even though all the $\beta$ receptors produce the same secondary messenger (cAMP) each type of receptor produces a different response. $\beta-1$ Receptors increase cardiac rate and force, relax gastrointestinal tract smooth muscle and are found mainly in heart muscle. $\beta-3$ Receptors encourage lipolysis and are associated with fat cells. $\beta-2$ Receptors cause bronchodilation, vasodilatation, relaxation of visceral smooth muscle, hepatic glycolysis and muscle tremor. It has not yet been possible to produce a $\beta$-agonist which only stimulates one specific receptor type. $\beta$-Agonists are allocated to a group by their relative strength of action rather than by a specific individual action. Like 


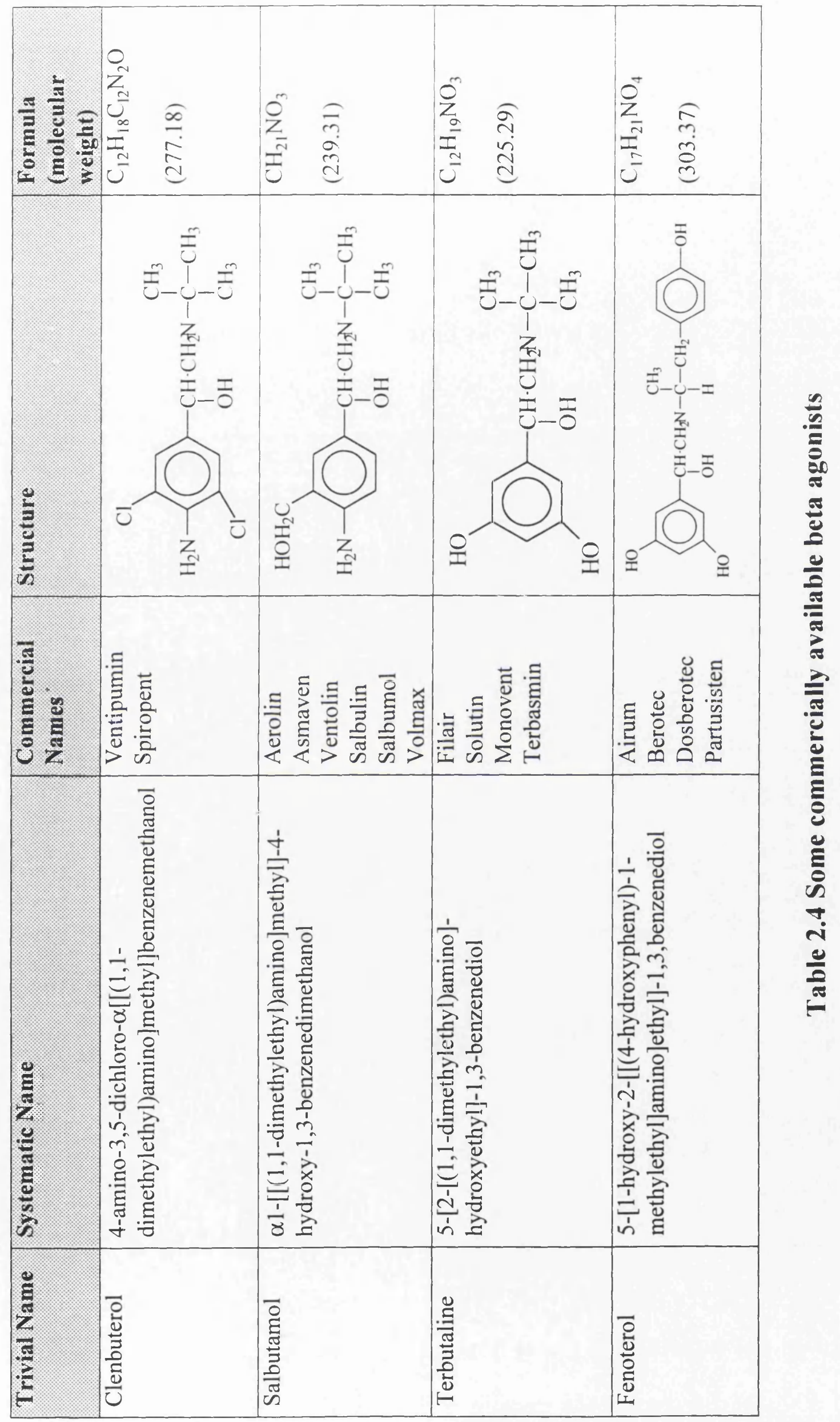


anabolic/androgenic steroids, compounds have been developed which maximise the desired effects and minimise, but not eliminate, the undesired effects.

As indicated above, all $\beta$-agonists stimulate the production of cAMP. Figure 2.12 illustrates the mechanism of action. All adrenoceptors are $G$ proteins. The term G protein simply means that the receptor interacts with the nucleotides guanidine triphosphate (GTP) and guanidine diphosphate (GDP). When the receptor is in its normal resting state, the G-protein exists as a trimer of $\alpha, \beta$ and $\gamma$ subunits with GDP bound to the $\alpha$ subunit. When a receptor is activated by binding to an agonist, the receptor combines with the $\alpha$ subunit and a molecule of GTP exchanges with the GDP. The GTP allows the $\alpha$ subunit to dissociate itself from the other two units and bind to the target enzyme, in this case adenylate cyclase, and allows the formation of the secondary messenger. The GTP is then hydrolysed to GDP by the $\alpha$-subunit and this then re-associates with the other two units of the trimer. The system then reverts to the resting state. 


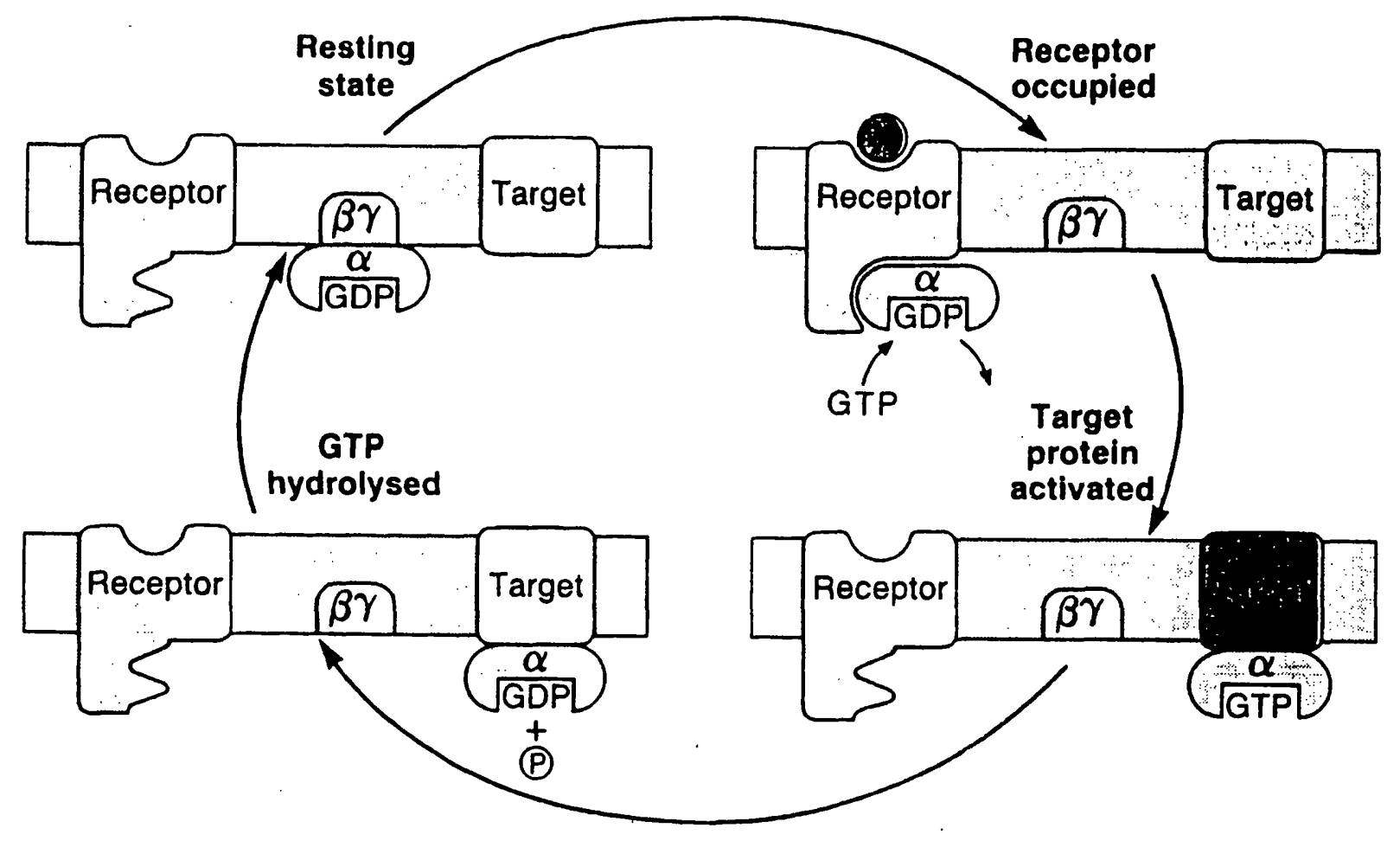

Figure 2.12 Mode of action of Beta agonists 


\section{Chapter 3 Analytical Techniques}

While it is possible to analyse biological matrices directly for some compounds of interest, for example headspace analysis for volatile components, it is more usual that some form of sample pre-treatment is required for an effective and sensitive analysis. In this study the majority of the analyses were accomplished using either liquid-liquid or solid phase extraction (SPE) pre-treatment prior to analysis by gas chromatography (GC) or gas chromatography-mass spectrometry (GC-MS). A discussion of the principles of these techniques is given below.

\section{Chromatography Theory.}

When a compound is subjected to a chromatographic process, it is partitioned between the mobile phase and the stationary phase. The distribution between these two phases can be expressed by the distribution constant $\left(K_{D}\right)$ which is defined as the ratio of the weight of solute in equal volumes of stationary and mobile phase:

$$
K_{D}=\frac{\text { Concentration per unit volume of stationary phase }}{\text { Concentration per unit volume of mobile phase }}=\frac{c_{s}}{c_{m}}
$$

As it passes along a chromatographic column, all compounds spend exactly the same amount of time in the mobile phase. The separation is caused by the compounds spending different amounts of time in the stationary phase.

The amount of time a component spends in the mobile phase can be determined by introducing into the system an unretained component. In gas 
chromatography, for example, methane is commonly used. The total retention time on the stationary phase can be expressed as

$$
t_{r}{ }^{\prime}=t_{r}-t_{m}
$$

where $t_{r}{ }^{\prime}$ is the stationary phase retention time or adjusted retention time, $t_{m}$ is the retention time of an unretained component, and $t_{r}$ is the time between injection and detection.

For a chromatographic process to be as sensitive as possible, it must deliver the solutes to the detector in as short as time as possible between the first and last molecule leaving the column. Thus the retention time on the column for all molecules of one particular compound is as similar as possible. The longer a compound spends on a column, i.e. the greater in $K_{D}$, the wider the band of solute becomes. This band widening can be influenced by many factors, including longitudinal diffusion.

The separation efficiency of a column is related to the extent to which a solvent band widens or lengthens relative to the retention time on the column and is measured in theoretical plates, a term taken from distillation technology. The number of theoretical plates $(n)$ is defined as:

$$
n=\frac{t_{r}{ }^{2}}{\delta}
$$

where $\delta$ is the standard deviation of the peak.

To ease calculations, if it is assumed that the peak is Gaussian, this equation can be rearranged as: 


$$
n=16\left(\frac{t_{r}}{w_{b}}\right)^{2}
$$

or

$$
n=5.54\left(\frac{t_{r}}{w_{h}}\right)^{2}
$$

where $w_{b}$ is the peak width at the base and $w_{h}$ is the peak width at half the height of the peak. These assumptions can be made because peak width at the base of a peak is $4 \delta$ and at half height is $2.354 \delta$. In practice, the calculation of theoretical plates is normally carried out using the peak width at half height, as it is normally easier to determine this value. If the adjusted retention time, $t_{r}{ }^{\prime}$, is used in the equations the value obtained is called the number of effective theoretical plates, $N$.

Column efficiency can also be expressed as the height equivalent to a theoretical plate, $h$, and is calculated by:-

$$
h=\frac{L}{n}
$$

where $L$ is the length of the column. Likewise, the height equivalent to an effective theoretical plate, $H$, is a calculated by substituting in $N$ for $n$ in the previous equation. The greater the column efficiency, the smaller the values of $h$ and $H . N$, $n, h$ and $H$ are all affected by the column temperature, column geometry, the nature of both the mobile and stationary phases, and the nature of the test compound. 
As stated above, all compounds spend the same amount of time in the mobile phase, $\left(t_{m}\right)$, but varying amounts of time in the stationary phase, $t_{r}{ }^{\prime}$. The partition ratio (or capacity factor), $k$, is defined as the amount of a solute in the stationary phase compared to the amount of solute in the mobile phase. This relationship is proportional to the amount of time a solute spends in the stationary phase $\left(t_{r}{ }^{\prime}\right)$ relative to the amount of time the solute spends in the mobile phase, $t_{m}$, and this can be expressed as:

$$
k=\frac{t_{r}{ }^{\prime}}{t_{m}}
$$

It is possible to calculate the difference between the theoretical plate number $(n)$ and the effective theoretical plate number $(N)$. This difference is a function of $k$ and can be expressed by:

$$
N=\left(\frac{k}{(k+1)}\right) n
$$

As the retention time increases, i.e. a large value of $k$, the value of $N$ approaches $n$.

It should be apparent that $k$ is related to $K_{D}$. This relationship depends on the relative availability of both the mobile phase and the stationary phase, i.e. the volumes of each component of the chromatographic system. This is called the column phase ratio and is given the symbol $\beta$

$$
\beta=\frac{V_{m}}{V_{s}}
$$

If the equation for the distribution constant $K_{D}$ is rearranged it can be expressed as 


$$
K_{D}=\frac{\text { Amount in the stationary phase / volume of the stationaryphase }}{\text { Amount in the mobile phase / volume of the mobile phase }}
$$

If this equation is multiplied out, the following is obtained

$$
K_{D}=\frac{\text { Amount in the stationary phase }}{\text { Amount in the mobile phase }} \times \frac{\text { Volume of the mobile phase }}{\text { Volume of the Stationary phase }}
$$

The second part of the equation is the column phase ratio, $\beta$, and the initial part of the equation is the partition ratio, $k$. Therefore,

$$
K_{D} \quad=\beta k
$$

\section{Separation of two components in a chromatographic system}

The ability of a chromatographic system to separate two components is termed resolution and is given the symbol $R_{s}$. This ability to separate peaks depends on the ratio of the retention times of the two components and the sharpness of the two peaks and is given the symbol $\alpha$. This value $\alpha$ is expressed as:

$$
\alpha=\frac{t_{r(2)}}{t_{r(1)}}
$$

Consequently, it can be seen that

$$
\alpha=\frac{k_{(2)}}{k_{(1)}}=\frac{K_{D(2)}}{K_{D(1)}}
$$

As the value of $\alpha$ decreases, so greater and greater numbers of theoretical plates are required to achieve separation.

The resolution of two peaks can be calculated from:

$$
R s=\frac{2\left(t_{r(2)}-t_{r(1)}\right)}{w_{b(1)}+w_{b(2)}}
$$


If the peaks are assume to be Gaussian, then the relationship between $w_{b}$ and $w_{h}$ can be used to the equation can be stated as:

$$
R s=\frac{1 \cdot 18\left(t_{r(2)}-t_{r(1)}\right)}{w_{h(1)}+w_{h(2)}}
$$

A resolution of 1.5 is required to provide base line separation of two Gaussian peaks. In practice, a greater degree of resolution is required to provide base line separation, as other chromatographic factors come into play, e.g. peak tailing and asymmetry 


\section{Mass Spectrometry.}

The mass spectrometer is used to give measurements of isotopic masses and abundances. Early work by $\mathrm{J} \mathrm{J}$ Thompson ${ }^{74}$ demonstrated the isotope forms of stable isotopes. There are several types of mass analyser available, two of which were used in this study.

A pre-requisite for analysis by mass spectrometry is the formation of ions. There are several means by which ions can be formed but the most common method is by using the electron impact source. The majority of work in this study was carried out using electron impact ionisation. This source was initially employed by Dempster $^{74}$ and further developed by Nier ${ }^{74}$. A schematic diagram of the electron impact source is shown in Figure 3.1. The sample, in the form of a vapour, passes through an electron beam. The electrons, formed by heating a filament (normally made of tungsten), are accelerated towards the electron trap. Some of the electrons pass through an opening in this plate into a chamber containing the vaporised sample. The gaseous sample molecules interact with the electrons and some of the molecules lose an electron to form positive ions. These ions may then further 'break up' or fragment. Approximately $0.1 \%$ of the sample molecules become ionised. These positively charged ions are repelled or expelled from the ion source by applying a small voltage to the repeller plate. The actual ionisation chamber is relatively gas tight to aid the ionisation of the sample molecules but the source housing and the analyser sections of the mass spectrometer must be kept under a high vacuum to prolong the life of the filament and the stability of the ions formed in the electron source. All areas of the electron source are kept heated to prevent the condensation of the sample vapour on the source. 


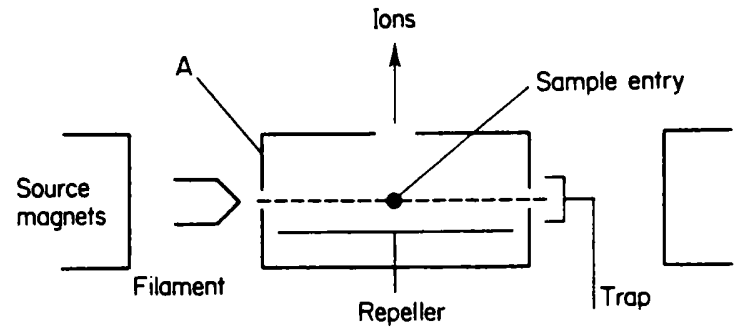

Figure 3.1 Schematic Diagram of an Electron Impact Source. 
The ions are then analysed by their mass to charge ratio. As stated above, there are several types of mass analyser available. The modes of operation of the two types of analyser used in this study were:

\section{1) Magnetic Sector Analyser. (Figure 3.2a)}

In this type on analyser, the ions, after exiting from the source come under the influence of the accelerating plates. These plates can be held at up to 10,000 volts and their purpose is to increase the kinetic energy of the ions. The kinetic energy gained by the ions is the same as the potential energy lost and can be summarised by:-

$$
z e V=\frac{m v^{2}}{2} \quad \text { Equation } 1
$$

where $e$ is the charge on an electron, $z$ is the number of the charges on the ion, $m$ is the mass of the ion, $V$ is the applied voltage and $v$ is the velocity of the ion. For the ion to reach the detector successfully, it must traverse a curved path of radius of curvature $r$, through a field of magnetic strength $B$. Equation 2 expresses the balance between the angular momentum and the centrifugal force caused by the magnetic field:

$$
\frac{m v^{2}}{r}=B z e v \quad \text { Equation } 2
$$

If the second equation is re-arranged it can be seen that:

$$
\frac{m v}{r}=B z e \quad \text { Equation } 3
$$

$m v$ is also known as momentum so it can be seen that a single magnetic sector instrument is in reality a momentum analyser.

If equations 1 and 2 are combined, the basic mass spectrometry equation is obtained 

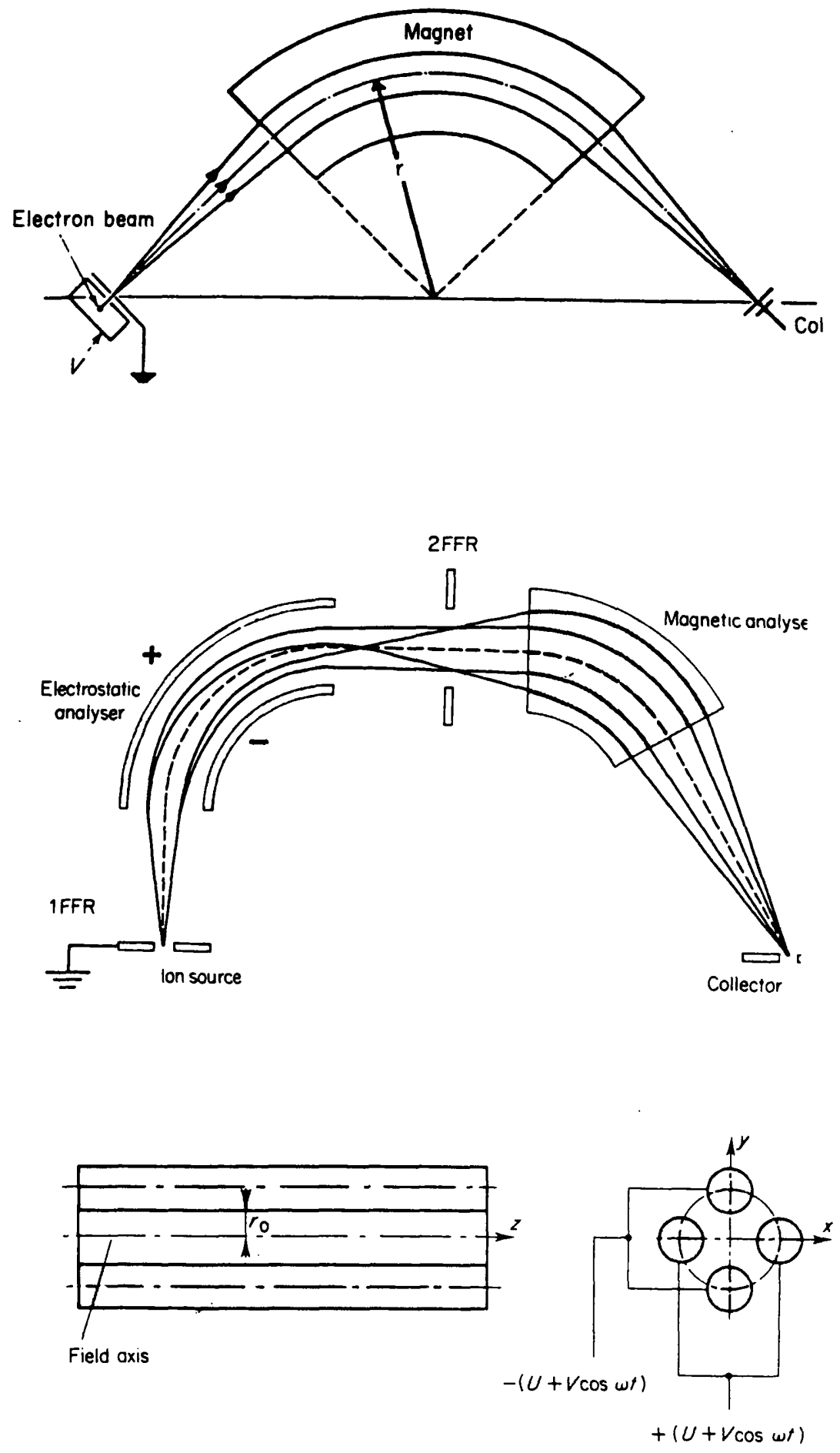

Figure 3.2 Various Types of Mass Spectrometers 


$$
\frac{m}{z}=\frac{B^{2} r^{2} e}{2 V} \quad \text { Equation } 4
$$

By varying the magnetic field strength $(B)$ or the voltage $(V)$ ions of different $m / z$ ratio can reach the detector.

\section{2) Double Focusing Mass Spectrometer. (Figure 3.2b)}

The resolution of a mass spectrometer can be defined as its ability to separate ions of adjacent mass numbers. The resolution necessary to separate two masses is described in equation 5 and illustrated in Figure 3.3:

$$
R=\frac{m}{\Delta m} \quad \text { Equation } 5
$$

where $R$ is the resolution, $m$ is the mass of an ion and $\Delta m$ is the difference in mass between the two ions. Resolution in mass spectrometry is comparable to resolution in chromatography, the closer two peaks are together, the greater the resolution required to baseline separation. Two peaks of equal intensity are considered to be separated by a valley of $10 \%$ of the height of either peak. At this point, the $10 \%$ valley consists of a contribution of $5 \%$ from each peak.

The higher the resolution, the greater the ability of a mass spectrometer to distinguish between two masses. A resolution of 580 is required to separate argon molecular weight 39.96239 from $\mathrm{C}_{3} \mathrm{H}_{4}$, molecular weight 40.03130. A resolution of 20600 is required to distinguish between ${ }^{13} \mathrm{C}^{12} \mathrm{C}_{6} \mathrm{H}_{7}$ xylene fragment ion weight 92.05813, and toluene $\mathrm{C}_{7} \mathrm{H}_{8}$, molecular weight 92.06260 . 
As stated above, a magnetic sector instrument is effectively a momentum analyser. If two ions have the same mass but a different translocational energy, they will not be brought to a focus at the same point on the detector. The spread of this translocational energy limits the resolution of the instrument. This limitation can be overcome by the introduction of an electrostatic sector to the instrument.

An ion entering the electrostatic field travels along a path of radius $r$ such that the electrostatic force balances the centrifugal force. The equation of motion is

$$
\frac{m v^{2}}{r}=e z E \quad \text { Equation } 6
$$

where $E$ is the electrostatic field strength. Thus the radius of curvature of the ion is dependent on the energy of the ion, not the mass.

\section{Quadrupole Mass Analysers (Figure 3.2c)}

As the name suggests, a quadrupole analyser consists of four parallel rods of circular or hyperbolic cross section arranged symmetrically around a central axis. The rods lying opposite each other are coupled. The ions leave the source and drift down between the rods. By applying a combination of radio frequency and direct current to the rods, a mass separation occurs. For any one particular frequency, ions of a particular mass to charge ratio $(\mathrm{m} / \mathrm{z})$ will pass through the analyser and successfully reach the detector. Ions lighter than this particular mass will tend to strike the positive poles and heavier ones will strike the negative poles. Quadrupole 
mass analysers have the advantage that they can be significantly cheaper than sector instruments, but suffer from the lack of resolution. 


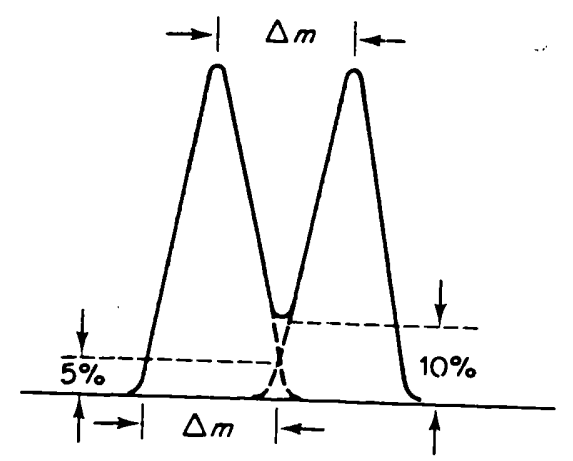

Figure 3.3 Definitions of Resolution 


\section{Interfacing a Mass Spectrometer and a Gas Chromatograph.}

For a mass spectrometer to work efficiently, it must be kept under the best vacuum possible. When interfaced to a gas chromatograph, this aim is compromised. The gas chromatograph actively puts molecules in the gaseous phase into the mass spectrometer. Depending on the nature of the column in the gas chromatograph and the flow rate of the carrier gas through the column, there are a variety of methods of sample introduction to a mass spectrometer, two of which are detailed below.

\section{The Jet Separator.}

If the column employed is a packed column or a capillary column with a relatively high flow rate (greater the $5 \mathrm{ml} / \mathrm{min}$ ) the pressure in the ion source and source housing is liable to be too high for efficient mass spectrometry. The jet separator is used to enrich the sample by removing as much of the carrier gas as possible while leaving the largest possible amount of the sample. The jet separator is such an enrichment device and was developed initially by Ryhage. For a detailed description of the jet separator see Chapman ${ }^{74}$. This technology is little used currently. The use of capillary columns has allowed a second route of sample introduction from a gas chromatograph.

\section{2.) Direct Coupling.}

If the chromatographic column has a low flow rate, normally a capillary column of internal diameter less the $0.32 \mathrm{~mm}$ and a flow rate below $3 \mathrm{ml} / \mathrm{min}$, it is possible to directly couple the column to the mass spectrometer. The high pumping speeds of modern pumps can remove the carrier gas efficiently enough to maintain a 
good vacuum and prevent significant interferences from any residual carrier gas. In direct coupling, the column can terminate only a few millimetres from the electron beam and so deliver almost all the sample to the ion source, increasing sensitivity of the analysis. The area between where the column leaves the gas chromatograph and enters the mass spectrometer is termed the transfer line. This transfer line must be kept hot. If not, material will condense out in the transfer line and efficient transfer of the analyses between the gas chromatograph and the mass spectrometer will not occur.

\section{Extraction Techniques}

As indicated above, it is not normally possible to analyse samples directly. Compounds of interest must usually be extracted from the matrix that they are in. This allows the sample to be concentrated and many components in the matrix to be removed which could interfere in the analysis. Two types of extraction procedure were used in this project.

\section{1). Liquid-Liquid Extraction.}

This is the traditional type of extraction where an aqueous sample is agitated with an immiscible organic phase, (e.g. ethyl acetate) and the compounds of interest leave the aqueous phase and enters the organic phase. At equilibrium, the ratio of compounds between the two phases is constant and this gives a number, called the distribution constant, $K_{d}$ and is defined as follows;

$$
K_{d}=\frac{c_{1}}{c_{2}}
$$

where $K_{d}$ is the distribution constant, $c_{1}$ is the amount in the organic phase and $c_{2}$ is the amount in the aqueous phase. 
This constant can be dependent on concentration but this only comes into play if the level of material is greater than $0.1 \mathrm{M}$. In order to hasten the attainment of equilibrium, the samples are normally agitated. If the samples are of low viscosity (e.g. water) and low volume (less than $100 \mathrm{ml}$ ) it has been shown that in test tubes as little as 50 shakes are necessary for the attainment of equilibrium. It is normal practice to leave the samples on a shaking platform of some form for around 10-15 minutes to ensure the attainment of equilibrium. It is also possible to manipulate the conditions in the aqueous phase to increase the selectivity of the extraction by the use of buffers and ion pairing reagents. Once this equilibrium has been established, the two layers are separated and the layer of interest is kept and subjected to either further manipulation or analysis.

\section{Solid Phase or Sorbent Extraction.}

Sorbent extraction is a physical extraction process that involves both a solid and liquid phase. A liquid sample is passed over the sorbent bed, which binds the compounds of interest reversibly. Once bound, interfering substances can be eluted from the sorbent bed by the use of carefully selected washes. These washes can be aqueous, organic or mixtures of aqueous and organic solvents. Once the majority of the interferences have been removed, the compounds of interest can be eluted form the sorbent bed by the use of an appropriate solvent. In general, the sorbent consists of functional groups attached to a chemically modified silica backbone. A wide variety of functional groups is available. One of the more common functional groups is $\mathrm{C18}$, an octadecyl straight chain hydrocarbon. It extracts the compounds of interest from the biological matrix primarily by non-polar interactions. It is the most non-polar sorbent available and is probably the least selective sorbent. It 
retains almost anything from an aqueous mixture. Polar and cation exchange mechanisms are also possible on a $\mathrm{C} 18$ column, the cation exchange properties arising from the activity of silanol groups within the silica backbone. The 'Sorbent Extraction Technology Handbook' provides a detailed review of the principles of sorbent or solid phase extraction, and the various functional groups available ${ }^{75}$. 


\section{Chapter 4 Analytical Techniques Practical}

This section details common analytical procedures employed in these studies. Additional relevant methodology is listed in each of the following sections as appropriate.

\section{Materials}

Steroid standards, deuterium-labelled testosterone standard, Helix pomatia digestive juice, creatinine assay kits, clenbuterol hydrochloride and heptafluorobutyric acid anhydride were purchased from Sigma Chemicals Ltd., Poole, Dorset, UK. ${ }^{3} \mathrm{H}$-Testosterone (specific activity $319 \mathrm{mCi} / \mathrm{mg}$ ) was purchased from Amersham International plc, UK. ${ }^{3} \mathrm{H}$-Corticosterone, ${ }^{3} \mathrm{H}-$ dehydroepiandrosterone sulphate and ${ }^{3} \mathrm{H}$-oestradiol glucuronide were purchased from New England Nuclear, USA. Methoxyamine hydrochloride, trimethylsilylimidazole (TMSI) N-methyl-N-(trimethylsilyl)-trifluoroacetamide (MSTFA) and hexamethyldisilazane (HMDS) were purchased from Pierce

Warrender, Chester, UK. Insta-gel scintillation fluid and Lipidex-5000 were purchased from Canberra Packard Ltd., UK. Laurabolin (nandrolone laurate) was obtained from Intervet UK Ltd, Cambridge, UK. All other solvents and reagents employed were of analytical grade or higher.

\section{Gas Chromatography-Mass Spectrometry System 1.}

The gas chromatograph was a Hewlett-Packard model HP5890 fitted with a fused silica column (CP-Sil 5, 25m x 0.32mm i.d., df $0.4 \mu \mathrm{m}$ ). Samples were injected using a Hewlett-Packard model 7673 autoinjector and a split/splitless injector fitted with a goosenecked liner. The latter was operated in the splitless mode for $0.7 \mathrm{~min}$ after injection. The mass spectrometer used was a VG Analytical model $70-250 \mathrm{~S}$ double focusing instrument. The delivery line from the GC to the 
MS was held at $280^{\circ} \mathrm{C}$. The carrier gas employed was helium with a linear velocity of $30 \mathrm{~cm} / \mathrm{sec}$. Individual gas chromatograph and mass spectrometer parameters are listed with the appropriate section.

\section{Gas Chromatography-Mass Spectrometry System 2.}

System 2 consisted of a Carlo Erba Gas Chromatograph model GC8000 containing a DB-5-MS fused silica column $(20 \mathrm{~m} \times 0.18 \mathrm{~mm}, \mathrm{df} 0.18 \mu \mathrm{m})$ attached to a Fisons Instruments MD800 quadrupole mass spectrometer. The carrier gas used was helium, with a linear velocity of $25 \mathrm{~cm} / \mathrm{second}$. The injector temperature was held at $250^{\circ} \mathrm{C}$. The delivery line between the gas chromatograph and the mass spectrometer was held at $270^{\circ} \mathrm{C}$. All samples were injected using an AS800 autosampler with the gas chromatograph operating in the splitless mode for one minute after injection. For the relevant mass spectrometer and gas chromatograph parameters, see the appropriate text.

\section{Scintillation Counting}

All scintillation counting used a Canberra Packard 2200CA Tri-Carb liquid scintillation analyser. All samples were counted for five minutes.

\section{Extraction of Urinary Steroids to Establish the Typical Urinary Steroid Profile of the Racing Greyhound}

The following method was used, based on procedures published by Shackleton et al. ${ }^{76,77,78}$.

\section{Extraction and Hydrolysis}

Urine $(10 \mathrm{ml})$ was centrifuged at $2500 \mathrm{rpm}$ for 10 minutes to remove any particulate material. The urine was applied to a C18 Sep-Pak ${ }^{\circledR}$ cartridge which had been conditioned with methanol $(10 \mathrm{ml})$ and distilled water $(10 \mathrm{ml})$. The cartridge was washed with a further portion of distilled water $(10 \mathrm{ml})$ and eluted with 
methanol $(5 \mathrm{ml})$. The extract was taken to dryness under a stream of nitrogen at $60^{\circ} \mathrm{C}$. Once dry, the residue was suspended in sodium acetate buffer $(\mathrm{pH} 4.6,0.2 \mathrm{M}$, $20 \mathrm{ml}$ ) and Helix pomatia digestive juice extract containing approximately 100,000 units of glucuronidase activity and 8,000 units of sulphatase activity was added. This was incubated in a water bath at $55^{\circ} \mathrm{C}$ for 3 hours or at $37^{\circ} \mathrm{C}$ for 24 hours.

Following incubation, the sample was re-extracted through the same C18 Sep-Pak ${ }^{\circledR}$ cartridge previously used following the same procedure described above. The extract was taken to dryness under a stream of nitrogen at $60^{\circ} \mathrm{C}$ and the residue was dissolved in cyclohexane:ethanol $(4: 1 \mathrm{v} / \mathrm{v}, 5 \mathrm{ml})$.

\section{Sample Purification}

Sephadex LH-20 (1g) was equilibrated in an excess of cyclohexane:ethanol $(4: 1 \mathrm{v} / \mathrm{v})$. Once equilibrated, the gel was transferred to a chromatography column $(20 \mathrm{~cm} \times 1 \mathrm{~cm}$ i.d.) with a reservoir $(50 \mathrm{ml})$ at the top and a sintered glass plate above a stopcock at the lower end. The sample was loaded onto the Sephadex column, taking care not to allow the Sephadex to dry out. The column was eluted with a further portion of cyclohexane:methanol $(4: 1 \mathrm{v} / \mathrm{v}, 45 \mathrm{ml})$. This fraction contained the steroids while the more polar components of the matrix were retained on the column. The column was regenerated by washing with methanol $(75 \mathrm{ml})$ before reconditioning it with cyclohexane:ethanol $(4: 1 \mathrm{v} / \mathrm{v})$ prior to loading the next sample.

The fractions containing the eluted steroids were concentrated by rotary evaporation, transferred to vials and stored at $-20^{\circ} \mathrm{C}$ until required for analysis.

\section{Formation of Methoxime Derivatives}

Each extract was dried under a stream of nitrogen and a solution of methoxyamine hydrochloride $(2 \% \mathrm{w} / \mathrm{v}$ in pyridine, $50 \mathrm{ml})$ was added and incubated at $60^{\circ} \mathrm{C}$ for $60 \mathrm{~min}$.

\section{Formation of Trimethylsilyl Derivatives}


TMSI $(50 \mathrm{ml})$ was added and the mixture heated for 16 hours at $100^{\circ} \mathrm{C}$.

\section{Removal of Excess Derivatisation Reagents}

To remove the excess derivatisation reagents the mixture was passed through a short column of Lipidex-5000 equilibrated with cyclohexane:pyridine:HMDS $(98: 1: 1, \mathrm{v} / \mathrm{v} / \mathrm{v})$. Once the sample was applied, the column was eluted with a further portion of cyclohexane:pyridine:HMDS $(2 \mathrm{ml})$. This fraction was collected and evaporated under a stream of nitrogen at $60^{\circ} \mathrm{C}$ and the residue re-dissolved in hexane $(100 \mu l)$ and aliquots $(1 \mu 1)$ analysed by GC-MS.

\section{GC-MS Conditions}

All GC-MS was carried out employing system 1. The injector temperature was $280^{\circ} \mathrm{C}$ and the column oven was programmed from $60^{\circ} \mathrm{C}$, (held for $0.5 \mathrm{~min}$ ), at $20^{\circ} \mathrm{C} / \mathrm{min}$ to $200^{\circ} \mathrm{C}$, (held for $0.5 \mathrm{~min}$ ), then at $5^{\circ} \mathrm{C} / \mathrm{min}$ to $300^{\circ} \mathrm{C}$ (held for $15 \mathrm{~min}$ ). The MS was operated in full scan mode ( 1 second/decade, interscan time $0.3 \mathrm{sec}$ ) using electron impact ionisation at $30 \mathrm{eV}$.

\section{Metabolic Study 1: ${ }^{3}$ H-Testosterone}

\section{Dose Preparation}

Radioactive testosterone (ca $150 \mathrm{mCi}$ ) was dissolved in $60 \%$ aqueous ethanol. The dose homogeneity was checked by taking small known aliquots of the dose and assessing the radioactivity present. The coefficient of variance (c.v.) was determined and found to be acceptable (c.v. $=0.87 \%, \mathrm{n}=6$ ). The dose was sterilised by passing it through a Millix-OR $0.2 \mathrm{~mm}$ filter obtained from Millipore, and sealed in a sterile vial with a rubber septum.

\section{Dose Administration}

One male greyhound was dosed intravenously with $1 \mathrm{ml}$ of the prepared solution, injected into the jugular vein. The greyhound was housed in a steel 
metabolic cage to allow complete collections of urine and faeces to be made, and to prevent the radioactive contamination of the kennel environment.

\section{Sample Collection}

To facilitate the collection of blood samples, a cannula was inserted into the jugular vein of the animal under local anaesthetic prior to dosing. Blood samples were collected by allowing blood to pass through the cannula into the heparinised sample tubes and were separated into red blood cells and plasma by centrifugation. The plasma was removed for analysis. Separate urine collections were made at $8 \mathrm{~h}$, $24 \mathrm{~h}, 48 \mathrm{~h}, 72 \mathrm{~h}$ and $120 \mathrm{~h}$ post dose into pre-weighed containers. Faeces were collected when possible.

\section{Sample Analysis}

Portions of the plasma were taken and made up to $1 \mathrm{ml}$ where necessary with water. Scintillant, (Insta-gel, $10 \mathrm{ml}$ ) was added and the mixture shaken. The radioactivity present was determined by scintillation counting for $5 \mathrm{~min}$. The total weight of urine excreted was determined. A portion of the sample (ca. 1g) was weighed into a scintillation vial and scintillant (Insta-gel, 10ml) was added. The radioactivity present was determined by scintillation counting for $5 \mathrm{~min}$.

\section{Metabolic Study 2: ${ }^{2} \mathrm{H}_{3}$-Testosterone}

\section{Dose Preparation}

${ }^{2} \mathrm{H}_{3}$-testosterone $(8 \mathrm{mg}$ ) was dissolved in $60 \%$ aqueous ethanol. The solution was filtered through a Millix-OR $0.2 \mathrm{~mm}$ filter, and transferred to a sterile vial which was then sealed with a sterile rubber septum.

\section{Dose Administration}

One male greyhound, the same animal used for the radiolabelled testosterone experiment, was dosed intravenously with $1 \mathrm{ml}$ of the prepared solution, injected into the jugular vein. 


\section{Sample Collection}

Blood samples were taken from the jugular vein by syringe and transferred to heparinised blood tubes for separation into red blood cells and plasma. Urine samples were collected by a kennel nurse in a bowl during exercise periods.

\section{Sample Analysis}

This extraction method is based on a procedure by Belanger et al. ${ }^{79}$.

Ethanol $(5 \mathrm{ml})$ was added to the plasma sample $(1 \mathrm{ml})$ and gently mixed for 5 minutes. The sample was centrifuged $(2500 \mathrm{rpm}, 5 \mathrm{~min}$.) and the supernatant removed. The pellet was washed with a further portion of ethanol $(5 \mathrm{ml})$, centrifuged and the supernatant was removed and combined with the supernatant already obtained. The combined extracts were taken to dryness under a stream of nitrogen at $60^{\circ} \mathrm{C}$. To each sample, internal standard, $17 \beta$-trenbolone was added. The dry residue was suspended in methanol:water $(5: 95 \mathrm{v} / \mathrm{v}, 2 \mathrm{ml}$, solution A). A C18 Sep-Pak ${ }^{\circledR}$ cartridge was conditioned with methanol $(10 \mathrm{ml})$, distilled water $(10 \mathrm{ml})$ and solution $\mathrm{A}(10 \mathrm{ml})$. The sample was loaded onto the column and washed with solution A $(10 \mathrm{ml})$. The steroids were eluted from the column with methanol:water $(85: 15 \mathrm{v} / \mathrm{v}, 3.5 \mathrm{ml}$,).

\section{Sample Derivatisation}

The extract from the Sep-Pak ${ }^{\circledR}$ cartridge was taken to dryness under a stream of nitrogen at $60^{\circ} \mathrm{C}$. Trimethylsilyl derivatives were formed by adding MSTFA $(50 \mu \mathrm{l})$ and incubating at $60^{\circ} \mathrm{C}$ for $15 \mathrm{~min}$.

\section{Gas Chromatography Parameters}

All GC-MS work was carried out using GC-MS system 1. The injector temperature was $280^{\circ} \mathrm{C}$. The column oven was programmed from $100^{\circ} \mathrm{C}$, (held for $0.5 \mathrm{~min}$ ), at $10^{\circ} \mathrm{C} / \mathrm{min}$ to $300^{\circ} \mathrm{C}$ (held for $15 \mathrm{~min}$ ). Aliquots $(1 \mu \mathrm{l})$ were injected 
using a Hewlett-Packard model 7673 autoinjector.

\section{Mass Spectrometry Parameters}

The ions monitored and the dwell times are listed in Table 4.1. The ratio of ${ }^{2} \mathrm{H}_{3}$-testosterone to ${ }^{2} \mathrm{H}_{0}$-testosterone was calculated from the relative areas under selected ion chromatograms for their molecular ions of ${ }^{2} \mathrm{H}_{3}$-testosterone and ${ }^{2} \mathrm{H}_{0}$ testosterone.

\section{Metabolic Study 3: Nandrolone (19-nortestosterone)}

\section{Dosing}

Two dogs were dosed intramuscularly with $1 \mathrm{ml}$ of Laurabolin, a commercial preparation containing $50 \mathrm{mg}$ of nandrolone laurate per $\mathrm{ml}$ of oily solution.

\section{Sample Collection}

Blood samples (approximately $10 \mathrm{ml}$ ) were collected in heparinised sample tubes from either the jugular vein or the saphenous vein both pre-dose and at known time intervals post dose. Plasma was obtained by centrifuging the collected blood samples at $2500 \mathrm{rpm}$ for 10 minutes. Urine samples were collected as and when produced by the greyhound. All samples were stored at $-20^{\circ} \mathrm{C}$ until required for analysis

\section{Sample Extraction}

Plasma samples were extracted as for the ${ }^{2} \mathrm{H}_{3}$-testosterone samples using ${ }^{2} \mathrm{H}_{3}$-testosterone as the internal standard.

Urine samples were extracted using Bond-Elut ${ }^{\circledR} \mathrm{C} 18$ cartridges containing $500 \mathrm{mg}$ of sorbent. Following the addition of ${ }^{2} \mathrm{H}_{3}$-testosterone as an internal standard, the urine sample $(10 \mathrm{ml})$ was loaded on a cartridge which had been conditioned with methanol $(5 \mathrm{ml})$, water $(5 \mathrm{ml})$ and methanol:water $(5: 95 \mathrm{v} / \mathrm{v}, 5 \mathrm{ml})$. After loading the sample, the cartridge was washed with methanol:water $(5: 95 \mathrm{v} / \mathrm{v}$, 
$5 \mathrm{ml})$ and eluted with methanol:water $(80: 20 \mathrm{v} / \mathrm{v}, 5 \mathrm{ml})$. This extract was taken to dryness under a stream of nitrogen at $60^{\circ} \mathrm{C}$ and re-dissolved in sodium acetate buffer (pH 4.6, 0.2M, $20 \mathrm{ml}$ ). Helix pomatia digestive juice extract containing approximately 100,000 units of glucuronidase activity and 8,000 units of sulphatase activity was added and the mixture was incubated in a water bath at $37^{\circ} \mathrm{C}$ for 16 hours. The samples were then re-extracted through the $\mathrm{C} 18$ cartridges used previously using the same methodology.

\section{Sample Derivatisation}

Sample derivatisation was based on a method published by Ehrsson et al ${ }^{80}$. The extracts obtained were taken to dryness under a stream of nitrogen at $60^{\circ} \mathrm{C}$ and dissolved in toluene $(0.5 \mathrm{ml})$. Triethylamine $(0.05 \mathrm{M}$ in toluene, $100 \mu \mathrm{l})$ and heptafluorobutyric acid anhydride $(100 \mu \mathrm{l})$ were added and the resulting mixture heated at $100^{\circ} \mathrm{C}$ for 15 minutes. Following incubation, the solvent was removed under a stream of nitrogen at $60^{\circ} \mathrm{C}$ and the residue re-dissolved in toluene $(100 \mu \mathrm{l})$, prior to analysis GC-MS.

\section{GC-MS Parameters}

The gas chromatography parameters employed were the same as for ${ }^{2} \mathrm{H}_{3}$ testosterone. The mass spectrometer was employed in the selected ion recording mode, monitoring the ions listed in Table 4.2.

\section{Creatinine Measurements}

The levels of creatinine in each urine sample were determined with a commercial kit according to the manufacturer's instructions, using a suitable dilution of the urine ${ }^{81}$.

The diluted urine sample, blank or standard $(0.3 \mathrm{ml})$ was added to a plastic cuvette along with the supplied alkaline picrate solution $(3 \mathrm{ml})$ and allowed to stand at room temperature for 10 minutes. The absorbance of the standard and blank solutions 
were measured against the blank at $500 \mathrm{~nm}$. Acid reagent $(0.1 \mathrm{ml})$ was added to each cuvette, mixed thoroughly and allowed to stand for five minutes. A second reading of each sample was taken. The concentration of creatinine in each samples was determined using the following formula:

$$
\frac{\text { Initial } A_{\text {test }}-\text { Final } A_{\text {test }}}{\text { Initial } A_{\text {std }}-\text { Final } A_{\text {std }}} * \text { Std Conc }
$$

where $A_{\text {test }}$ is the absorbance of the urine sample, $A_{\text {std }}$ is the absorbance of the standard creatinine solution and Std Conc is the concentration of the standard. The correction factor for diluting the urine must also be applied. In general a thirty fold dilution of the greyhound urine sample was required (for humans, the kit recommends a 10 fold dilution). Results were expressed as $\mathrm{mg} / \mathrm{ml}$. The assay was specified to be accurate up to $100 \mathrm{mg} /$ litre. 


\section{Metabolic Study 4: Boldenone (1,2-dehydrotestosterone)}

\section{Dosing}

Two dogs were dosed intramuscularly with $1 \mathrm{ml}$ of Venobol, a commercial preparation containing $25 \mathrm{mg}$ of boldenone undecylenate per $\mathrm{ml}$ of oily solution.

Sample collection was as for the ${ }^{2} \mathrm{H}_{3}$-testosterone experiment except that the samples were taken from the saphenous vein.

\section{Sample Extraction}

Plasma samples were extracted as for the ${ }^{2} \mathrm{H}_{3}$-testosterone samples using ${ }^{2} \mathrm{H}_{3}$-testosterone as the internal standard.

Urine samples were extracted as for nandrolone

\section{Sample Derivatisation}

The extract from the Sep-Pak ${ }^{\circledR}$ cartridge was taken to dryness under a stream of nitrogen at $60^{\circ} \mathrm{C}$. Trimethylsilyl derivatives were formed by adding MSTFA $(50 \mu \mathrm{l})$ and incubating at $60^{\circ} \mathrm{C}$ for $15 \mathrm{~min}$.

\section{GC-MS Parameters}

All GC-MS work was carried out using GC-MS system 1 . The gas chromatography parameters employed were the same as for ${ }^{2} \mathrm{H}_{3}$-testosterone. The mass spectrometer was employed in the selected ion recording mode, monitoring the ions listed in Table 4.3.

\section{Creatinine Measurements}

As for nandrolone.

\section{Metabolic Study 5 Clenbuterol}

\section{Dose Preparation and Dosing}


A known weight of clenbuterol (approximately $2 \mathrm{mg}$ free base) was dispensed into a hard gelatine capsule and the remainder of the volume of the capsule was filled with lactose. One capsule was administered to one dog and one bitch by placing the capsule in the mouth of the greyhound and holding the greyhounds muzzle until the capsule was swallowed.

\section{Sample Collection.}

As for the studies with nandrolone.

\section{Sample Extraction}

A liquid-liquid extraction technique based on previously published methodology was used ${ }^{8}$. Plasma or urine samples ( $1 \mathrm{ml}$ or $2 \mathrm{ml}$ respectively) and internal standard (salbutamol) were placed in a test tube with sodium hydroxide buffered at $\mathrm{pH} 12$ with sodium hydrogen carbonate $(2 \mathrm{M}, 1 \mathrm{ml})$. Ethyl acetate $(6 \mathrm{ml})$ was added and the samples were placed on a rock and roll table for 15 minutes. The upper layer was decanted and the ethyl acetate extraction repeated. The combined ethyl acetate layers were back extracted into sulphuric acid $(0.2 \mathrm{M}, 1 \mathrm{ml})$ for 10 minutes. After centrifugation (2000g, $10 \mathrm{~min}$ ) the upper layers were discarded. Ethyl acetate/hexane $(2: 1,4 \mathrm{ml})$ was added and the acid layer was extracted for 5 minutes. The organic layer was discarded. Sodium hydroxide $(2 \mathrm{M}, 1 \mathrm{ml})$ was added to make the extract alkaline and ethyl acetate $(4 \mathrm{ml})$ was added and the samples extracted for 15 minutes. The upper organic layer was transferred to a clean $3 \mathrm{ml}$ vial and stored at $4^{\circ} \mathrm{C}$ until required for analysis.

\section{Sample Derivatisation}

The extracts were taken to dryness at $60^{\circ} \mathrm{C}$ under nitrogen and BSTFA with 
$1 \%$ TMCS $(50 \mu 1)$ was added. The samples were incubated at $80^{\circ} \mathrm{C}$ for 30 minutes prior to analysis as described below.

\section{Creatinine Measurements}

As for nandrolone.

\section{GC-MS Parameters}

GC-MS System 2 was used for the analysis of the biological extracts. The injector temperature was $250^{\circ} \mathrm{C}$ and the column oven was programmed from $100^{\circ} \mathrm{C}$ (held for 1 minute) at $10^{\circ} \mathrm{C}$ per minute to $300^{\circ} \mathrm{C}$ (held for 5 minutes). The delivery line between the Gas chromatograph and the mass spectrometer was held at $270^{\circ} \mathrm{C}$. The instrument was operated in the selected ion monitoring mode and the ions monitored are listed in Table 4.4. 


\begin{tabular}{|c|c|c|}
\hline Compound & Ions $(\mathrm{m} / \mathrm{z})$ & Dwell time (ms) \\
\hline $17 \beta$-Trenbolone & 327.1780 & 5 \\
& 342.2015 & 5 \\
\hline${ }^{2} \mathrm{H}_{0}$-Testosterone & 345.2250 & 50 \\
& 360.2485 & 50 \\
\hline${ }^{2} \mathrm{H}_{3}$-Testosterone & 348.2438 & 50 \\
& 363.2673 & 50 \\
\hline
\end{tabular}

Table 4.1: Selected ion recording parameters used for the analysis of selected steroids as their trimethylsilyl derivatives. 


\begin{tabular}{|l|c|c|}
\hline Compound & Ions (m/z) & Dwell time (ms) \\
\hline Testosterone/ & 665.1373 & 50 \\
Epitestosterone & 680.1608 & 50 \\
\hline Nandrolone & 666.1451 & 50 \\
\hline${ }^{2} \mathrm{H}_{3}$-Testosterone & 668.1561 & 10 \\
& 683.1795 & 10 \\
\hline Etiocholanolone/ & 471.1770 & 50 \\
Androsterone & 486.2005 & 50 \\
\hline
\end{tabular}

Table 4.2: Selected ion recording parameters used for the analysis of selected steroids as their heptafluorobutyrate derivatives. 


\begin{tabular}{|l|c|c|}
\hline Compound & Ions (m/z) & Dwell time (ms) \\
\hline Testosterone/ & 345.2250 & 50 \\
Epitestosterone & 360.2485 & 50 \\
\hline Boldenone & & 50 \\
\hline${ }^{2} \mathrm{H}_{3}$-Testosterone & 348.2438 & 10 \\
& 363.2673 & 10 \\
\hline
\end{tabular}

Table 4.3: Selected ion recording parameters used for the analysis of selected steroids as their trimethylsilyl derivatives. 


\begin{tabular}{|l|c|c|}
\hline Compound & Ions & Dwell Time (ms) \\
\hline Clenbuterol & 86.10 & 50 \\
& 262.03 & 50 \\
& 264.02 & 50 \\
\hline Salbutamol (Internal & 86.10 & 50 \\
Standard) & 369.17 & 50 \\
\hline
\end{tabular}

Table 4.4: Selected Ion Recording Parameters used for the Analysis of Clenbuterol and Salbutamol as their Trimethylsilyl Derivatives 


\section{Chapter 5 Elucidation of Endogenous Steroid Biochemistry of the Racing Greyhound}

\section{Introduction}

The detection of exogenous steroids in the greyhound or the detection of administration of steroids which are normally present in the greyhound both require background knowledge of greyhound steroid biochemistry. The reasons for this are almost self-evident but can be summarised as follows:

The steroid profile in biological specimens in most mammals is complex and likely to result in interferences in analyses targeting either endogenous or exogenous steroids. In addition, the steroid profile is related to the action of steroidal hormones and is therefore periodic or variable over several time-scales, both geophysical and biological within individual animals. Superimposed on this is interindividual variation

While the detection of exogenous substances may be easier and less ambiguously interpreted, the detection of supplementary endogenous hormones usually requires a different approach based on changes caused to the endogenous steroid profile resulting from the administration of the steroids. In humans, for example, administration of testosterone and its esters may be detected by changes induced in the testosterone:epi-testosterone ratio. The International Olympic Committee have adopted a threshold ratio of 6:1 as an indication of administration of testosterone. However, this has been subject to legal challenge in recent years, following the detection of individuals who apparently have normal ratios above this threshold. For these reasons, and also, because little has been published concerning steroids in the greyhound, it was considered essential to examine the normal steroid pattern in greyhounds before beginning controlled metabolic studies of administered substances.

The approach adopted drew on knowledge of the steroidal profile of other species, especially the human steroid profile, which has been extensively studied. 


\section{Steroid Standards}

Prior to the examination of the natural profile of steroids in greyhounds, a large number of pure steroid standards were derivatised to form both their trimethylsilyl (TMS) and methoxyamine-trimethylsilyl (MO-TMS) derivatives. The details of the derivatisation protocols and gas chromatograph-mass spectrometry parameters are given in Chapter 4. A list of some of the standards analysed along with the corresponding Kovats Indices are given in Table 5.1. These steroids included estrogens, androgens, progestagens and corticosteroids. Each sample was chromatographed three times and the quoted Kovats Index is the average of the three separate runs. The endogenous steroids selected to make up the standard mixture were determined by identifying steroids already noted as being present in the urine of other species, mainly human.

Initially, the amount of time required for the preparation of the TMS derivatives was far less than the reported sixteen hours at $100^{\circ} \mathrm{C}$. Some of the steroids, and in particular the corticosteroids, did not always derivatise effectively unless these reasonably aggressive conditions were used. This was probably due to steric hindrance around position 17 of the steroid nucleus. 
Steroid

Allo-THB

Allo-THF

$5 \alpha$-Androstan-3 $\beta, 17 \beta$-diol

5-Androstene-3 $\beta$, 16 $\alpha$-diol-17-one

Androstane

5-Androstene-3 $\beta, 16 \alpha, 17 \alpha$-triol

Cholestane

$5 \beta$-Cholestan- $3 \alpha$-ol

Corticosterone

Cortisol

$\alpha$-Cortolone

Ethynodiol

11- $\beta$-Hydroxyandrosterone

20- $\alpha$-Hydroxypregnenolone

11-Ketoaetiocholanolone

17- $\alpha$-Methyltestosterone

1 9-Norethisterone

Pregnandiol

$5 \beta$-Pregnane- $3 \alpha, 17^{\alpha}, 20 \alpha$-triol

5-Pregnene-3 $\beta, 17 \alpha, 20 \alpha$-triol

Proligestone

Secrosterone

Stanozolol

THB

THE

THF

Trenbolone
Retention Index

TMS Derivative

2560

$2549,2559,2641,2667$

2634

$2705,2729,2779$

2055

2397

2751

2877

\#

2084

2788

2038

2771

\#

\#

2706

2392

2480

2827

2827

\#

2920

2564

2902

2500

2766
Retention Index

MO-TMS derivative

2494, 2525+

2500, 2604+

$2711,2715+$

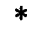

*

*

2838

$2967,2970+$

2940

2273

$2235,2280,2249^{\wedge}$

2717

2705

2755

*

2861, 2869+

$2849,2869+$

2486

2837

2541

2645

\section{Notes}

* Compound contains no carbonyl group. No methoxime derivative will therefor be formed.

\# Inconclusive data See text for details

$\wedge$ Differing levels of derivitisation can occur. Enolisation may occur.

+ Two peaks resulting form syn and anti isomers of methoximes. See text for details

\section{Table 5.1 Steroids and Kovats Indexes}


As well as the lack of formation of some of the derivatives described above, three other features were noted.

- The formation of two or more peaks. (Indicated by + in Table 5.1)

It was possible to form two or more derivatives for some of the compounds.

With the methoxime-TMS derivatives, this depends on the stereochemistry (synanti) of the compounds formed. Although problematic when trying to determine the complete steroid profile of the greyhound due to the creation of more peaks in the mass chromatogram, this 'double peak' formation can be utilised effectively. The ratio between the syn and anti isomers for a given compound and set of circumstances remains the same. In steroid analysis control by the Horseracing Forensic Laboratory, Newmarket, these two peaks are used as an additional check to confirm the identity of compounds. ${ }^{82}$ A compound will only be identified as being present if both the syn and anti isomers are different. This is a different approach to that of many International Olympic Committee Laboratories where an aggressive TMS derivatisation procedure results in the formation of enol TMS derivatives.

- The formation of two or more peaks following TMS derivitisation (Indicated by $\wedge$ in Table 5.1)

This results from the production of enol derivatives. The peaks at retention indices 2235 and 2280 correspond to bis-TMS derivatives while the peak at retention index 2249 results form the formation of a tris-TMS derivative. Further discussion of enolisation follows later in this chapter.

- The problematic formation of some derivatives. (Indicated by \# in Table 5.1) These derivatives should have been relatively easy to form with the temperatures and reaction time used. However, when it came to running these compounds on the GC-MS it was not possible to obtain retention time or mass spectra reliably from these compounds. It appeared as if these compounds would chromatograph acceptably initially, but within one or two runs, the ability to 
chromatograph these compounds disappeared. Changing the gas chromatograph injector liner to a clean one of the same type or different types, injector temperature, septum or chromatographic column were all attempted but none of these appeared to rectify the problem. Problems of this nature have been noted before ${ }^{83}$ but no satisfactory explanation had ever been determined. This problem may be instrument-specific as recently, two similar instruments from the same manufacturer have been compared and one has the ability to chromatograph the compounds while the other cannot.

\section{Elucidation of the background steroid profile of the racing greyhound}

Following determination of the behaviour of a large number of steroids, work progressed to the determination of the normal urinary steroid profile of the greyhound. The principal method utilised was the analytical approach detailed in Chapter 4 after the method by Shackelton et al. ${ }^{76,77}$. Initial experiments on human urine demonstrated that this analytical approach could be used to elucidate a steroid profile form a normal healthy male. However, all attempts to use this protocol to elucidate a similar profile in the racing greyhound met with limited success. The 'normal' profiles for the male and female racing greyhound are given in Figures 5.1 and 5.2.

\section{Male Racing Greyhound (Figure 5.1)}

It was not possible to establish the presence of any steroids in the urine of the male racing greyhound with certainty. Additional work was carried out and this is detailed later in this chapter.

\section{Female Racing Greyhound (figure 5.2)}

Unlike the male, it was possible to identify clearly the presence of steroids in the urine. These were 17ß-oestradiol (peak A), cortisol (peak B), tetrahydrocortisol 
荧

焉崖

올 뜨

王

豆

品

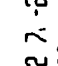

苕

우음

竬

品蒙

음

要哭

吕家

焉总

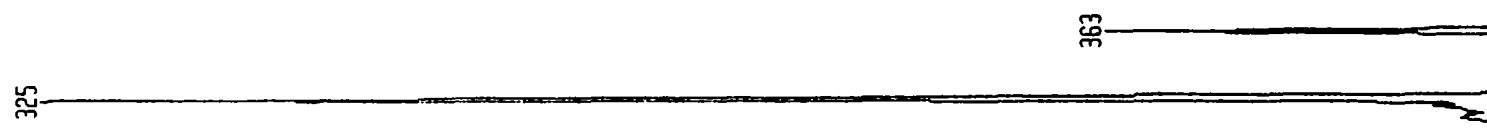

通

罳营

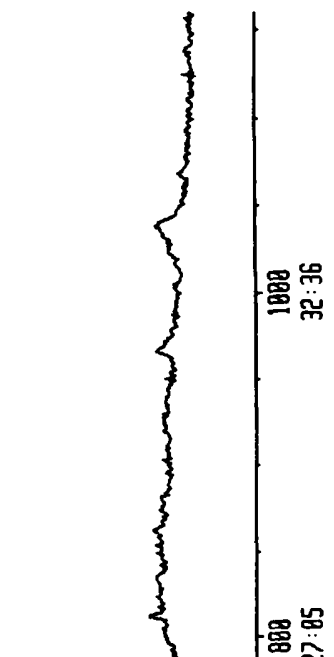

를

包 
䍃

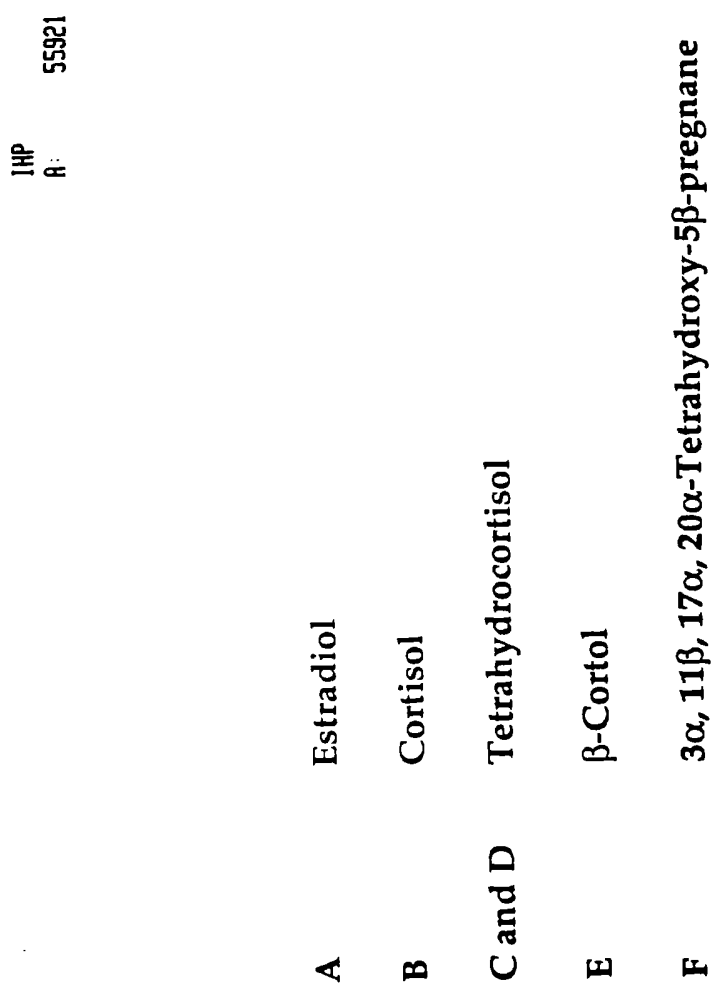

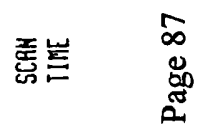

豆:

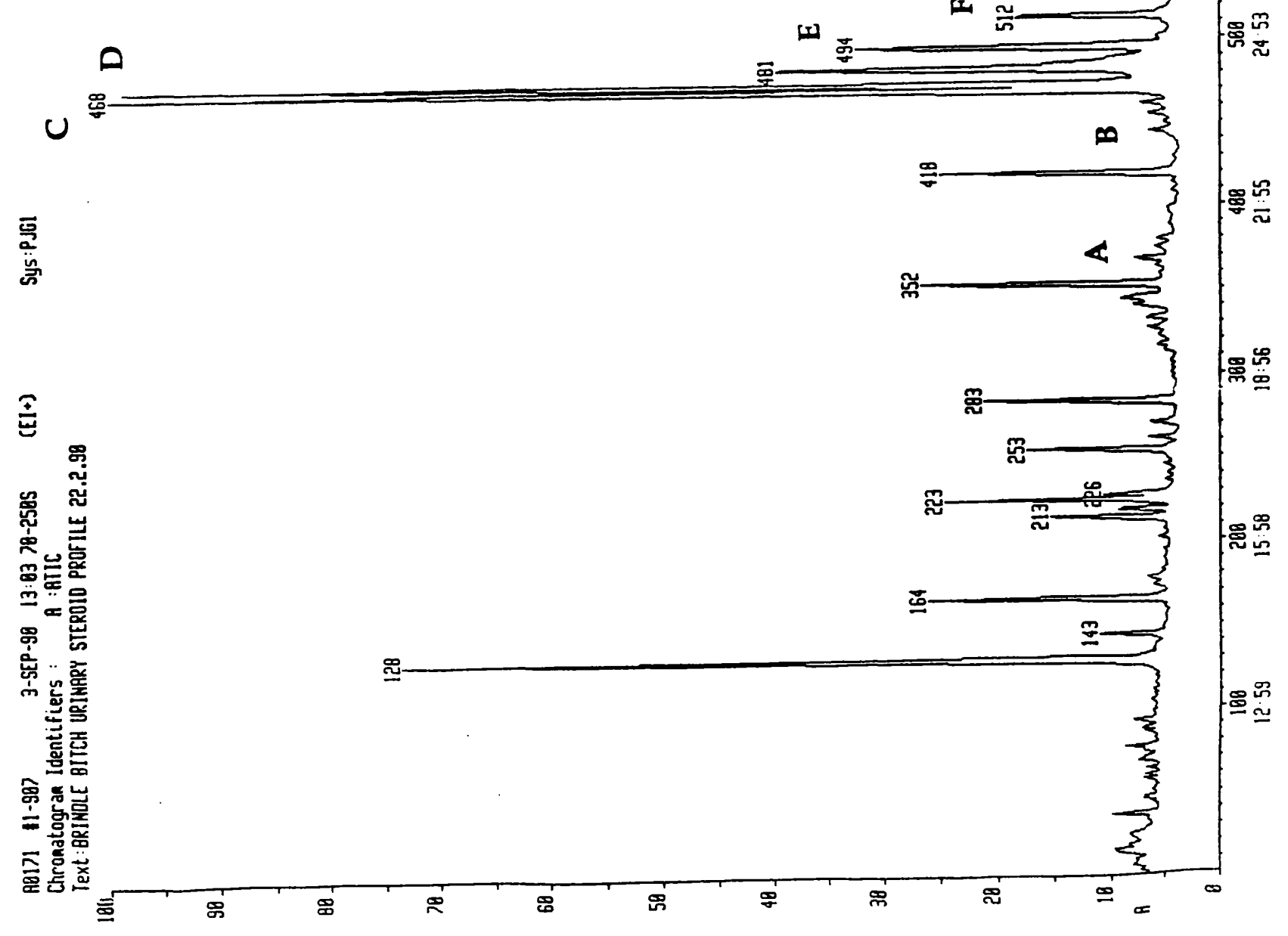


(peaks $\mathrm{C}$ and $\mathrm{D}$ ) and $3 \alpha, 11 \beta, 17 \alpha, 20 \alpha$-tetrahydro-5- $\beta$-pregnane (peak E). It is probable that some of the other peaks present in the chromatogram are also steroids but it was not possible to identify these peaks definitively. The two peaks for tetrahydrocortisol are due to the formation of diastereoisomers.

One of the few papers published on the steroid metabolism of greyhounds deals with the determination of corticosteroids in the plasma ${ }^{70}$. These authors examined the plasma levels of corticosteroids in the beagle and the greyhound. They reported mean plasma levels of 25.4 and $18 \mathrm{ng} / \mathrm{ml}$ of corticosteroids in beagles and greyhounds respectively. There appeared to be no significant difference between male and female greyhounds, with levels of 16.3 and 19.6 $\mathrm{ng} / \mathrm{ml}$ respectively. This assay only determines total corticosteroids in the sample and does not attempt to identify specific compounds. As the plasma levels of the corticosteroids appear to be similar, it would seem likely that the urinary levels would be similar. As said above, however, there do appear to be significant differences between the sexes.

These steroid profiles were obtained by extraction of pooled urine samples. It is possible that for the female animal, the animal was about to go into oestrus and the presence of the steroids in the urine indicates this. In particular, 17 $\beta$ oestradiol is associated with the control of oestrus in females.

It has been said that 'Humans are swimming in steroids, while other animals are not ${ }^{84}$. It is possible that for some unknown physiological purpose, humans have a far greater amount of steroids present in their system than do animals.

\section{Verification of the Analytical Protocol}

Following the failure to establish a steroid profile, the analytical protocol was examined stage by stage to determine if it was suitable for the analysis of exogenously-administered steroids in the racing greyhound.

The extraction protocol was examined using radiolabelled steroids. Urine was separately spiked with one of three steroids: tritiated corticosterone, tritiated 
17ß-oestradiol glucuronide or tritiated dehydroepiandrosterone sulphate (DHEA sulphate) at an approximate concentration of $200 \mathrm{ng} /$ millilitre and 100,000 dpm. The samples were taken through the entire extraction protocol detailed in Chapter 4 and the recoveries of the steroids are given in Table 5.2.

One free and two different conjugated steroids were used. Steroids of different types were used to represent all the target analytes. As can been seen from the table, the recoveries of all the steroids are greater than $56 \%$. Although not ideal, these recoveries are acceptable for a non-specific assay and clearly demonstrate that, if other steroids behave in a similar manner, then it should be possible to detect them using full scan mass spectrometry if they are present in any significant quantities. Once the suitability of the extraction assay had been determined, the effectiveness of the hydrolysis stage was examined. A high performance liquid chromatography system to separate $17 \beta$-oestradiol and $17 \beta$ oestradiol glucuronides was developed and is described below.

\section{Analytical Set-up}

An LKB 2150 pump was attached to a Hypersil C18 column ( $5 \mu$ m particle size, $250 \mathrm{~mm} \times 4.6 \mathrm{~mm}$ ). Detection was achieved using a Waters 490-MS UV/visible detector operated at $284 \mathrm{~nm}$ and the output of the detector was recorded on a Venture RE54120 strip chart recorder. The mobile phase was optimised for the separation of the two components and was a methanol water $\operatorname{mix}(80: 20, \mathrm{v} / \mathrm{v})$. A typical chromatogram showing the separation between the two components is shown in figure 5.3 .

$17 \beta$-Oestradiol glucuronide was spiked into hydrolysis buffer and incubated with enzyme as detailed in chapter 4. Following the incubation period proposed there was no $17 \beta$-oestradiol present in the extract. 
All this evidence combined suggested that there were little or no identifiable steroids present in the urine of the racing greyhound 


\begin{tabular}{|c|c|c|c|c|c|c|c|}
\hline Steroid & Concentration & $\begin{array}{l}\text { Mean \% } \\
\text { recovery }\end{array}$ & $\begin{array}{l}\text { Upper } \\
\text { recovery }\end{array}$ & $\begin{array}{l}\text { Lower } \\
\text { recovery }\end{array}$ & $\begin{array}{l}\text { Standard } \\
\text { Deviation }\end{array}$ & $\begin{array}{l}\text { Coefficient } \\
\text { of variation }\end{array}$ & $\begin{array}{l}\text { Number of } \\
\text { samples }\end{array}$ \\
\hline $\begin{array}{l}\text { 17ß-Oestradiol } \\
\text { glucuronide }\end{array}$ & $200 \mathrm{ng} / \mathrm{ml}$ & 76.6 & 81.6 & 72.9 & 3.96 & 5.1 & 6 \\
\hline Corticosterone & $200 \mathrm{ng} / \mathrm{ml}$ & 63.7 & 77.2 & 50.6 & 9.3 & 14.6 & 6 \\
\hline $\begin{array}{l}\text { DHEA } \\
\text { sulphate }\end{array}$ & $200 \mathrm{ng} / \mathrm{ml}$ & 56.5 & 68.3 & 44 & 9 & 16.4 & 6 \\
\hline
\end{tabular}

Table 5.2 Recovery of radiolabelled steroids extracted from pooled racing greyhound urine. 


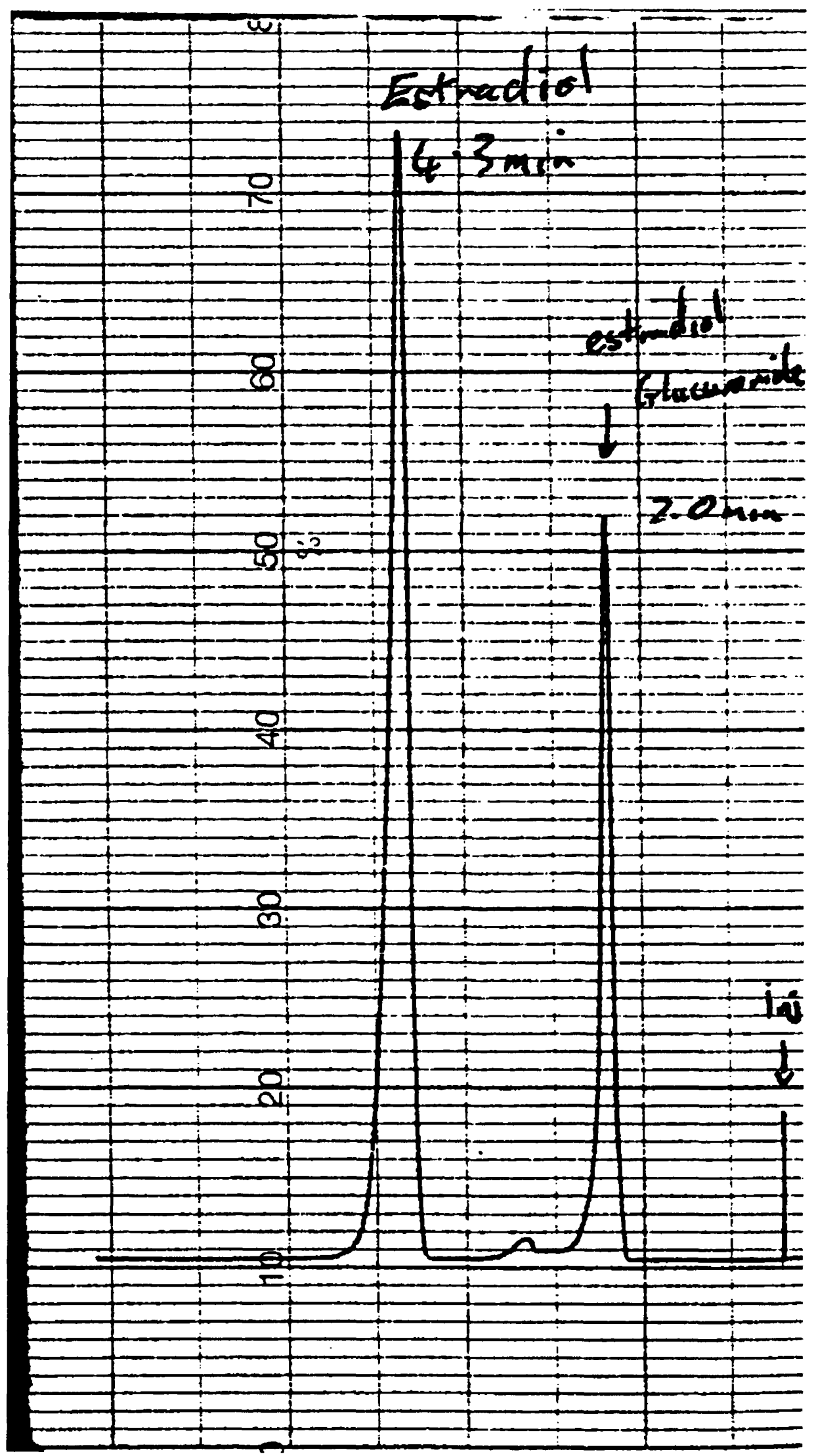

Figure 5.3 Sample chromatogram showing the separation of $17 \beta-$ Oestradiol and 17 $\beta$-Oestradiol glucuronide 


\section{Metabolism of Testosterone in the Racing Greyhound}

Following the unsuccessful attempts to establish a full urinary steroid profile in the male racing greyhound, it was decided to concentrate on the metabolism of testosterone and establish what was happening to one of the main androgenic steroids. This part of the study was divided into three parts:

The concentration of testosterone in the urine of untreated male greyhounds: A dual approach was taken to determine the concentration of testosterone in the urine of the male greyhound. Single samples were extracted and split into two equal portions. Portion one was analysed using a commercially available radioimmunoassay (RIA) kit and portion two was analysed by gas chromatography-mass spectrometry in the selected ion recording mode.

\section{Sample preparation:}

Concentrated hydrochloric acid $(2 \mathrm{ml})$ was added to greyhound urine $(5 \mathrm{ml})$ and incubated at $80^{\circ} \mathrm{C}$ for 15 minutes. Following hydrolysis, the samples were neutralised using sodium hydroxide and extracted with dichloromethane $(8 \mathrm{ml})$. After separation of the two layers the dichloromethane was split into two equal portions, $3 \mathrm{ml}$ in each portion. Portion A was analysed by RIA

\section{Radioimmunoassay analysis:}

The dichloromethane was taken to dryness under nitrogen at $60^{\circ} \mathrm{C}$. The extracts were re-suspended in buffer supplied with the RIA kit $(0.5 \mathrm{ml})$. Into clean, plastic tubes, samples controls and calibrators $(0.1 \mathrm{ml})$, ${ }^{125} \mathrm{I}$-testosterone and testosterone antiserum were added. This was allowed to sit at room temperature for 60 minutes. After incubation, Goat anti-rabbit globulin was added and the samples allowed to sit for a further 60 minutes. The samples were centrifuged at $2000 \mathrm{~g}$ for 20 minutes and the supernatant decanted. The remaining pellet was 
counted in a Cobra Gamma counter (supplied by Canberra Packard) for 1 minute. Concentrations of testosterone in the samples were calculated from a prepared calibration curve.

\section{GC-MS analysis}

Internal standard (androstane, $500 \mathrm{ng} / \mathrm{ml}$ ) was added to each sample. The dicholoromethane was taken to dryness under nitrogen at $60^{\circ} \mathrm{C}$ and the samples derivatised as described in chapter 4 for methoxime/TMS derivatives. Analysis was carried out monitoring the ions listed in table 5.3. A calibration curve was constructed (correlation coefficient of 0.9901 ) using the ratio of the areas under the curves of $\mathrm{m} / \mathrm{z} 389$ (testosterone) and $\mathrm{m} / \mathrm{z} 260$ (androstane).

\section{Creatinine analysis:}

As with almost all the experiments in this study, it was not possible to have complete urine collections. To try and standardise the urine concentrations and correct for variations in diurnal urine volume, creatinine concentrations were determined in all samples analysed as detailed in chapter 4.

\section{Testosterone concentration by RIA}

Figures $5.4 \mathrm{a}$ and $5.4 \mathrm{~b}$ show the concentrations of testosterone in urine samples taken from two separate dogs. One line represents the raw data and the other is corrected for creatinine concentration. As can be seen form the graphs, in most cases when the results are corrected for creatinine concentration, the levels of testosterone in the urine samples are far more consistent. There are, however, differences in the concentration of testosterone per milligramme of creatinine between the two animals and a far larger population of animals should be studied to determine normal ranges. 


\begin{tabular}{|l|l|}
\hline Compound & Ions Monitored \\
\hline Androstane & 245.2269 \\
& 254.9856 (lock mass) \\
& 260.2504 \\
\hline Testosterone & 358.2566 \\
& 380.9160 \\
& 389.2750 \\
\hline
\end{tabular}

Table 5.3 Ions monitored for the determination of testosterone in urine. 


\section{Testosterone concentration by GC-MS}

Analysis of the second portion of the extracts by GC-MS was unsuccessful. A significant interfering peak sat over the testosterone peak making any meaningful interpretation of the concentration of testosterone impossible. The result demonstrated clearly that the solid phase extractions clean up procedures detailed in Chapter 4 were required to obtain meaningful data. Due to lack of sufficient sample, it was not possible to repeat the above samples. 


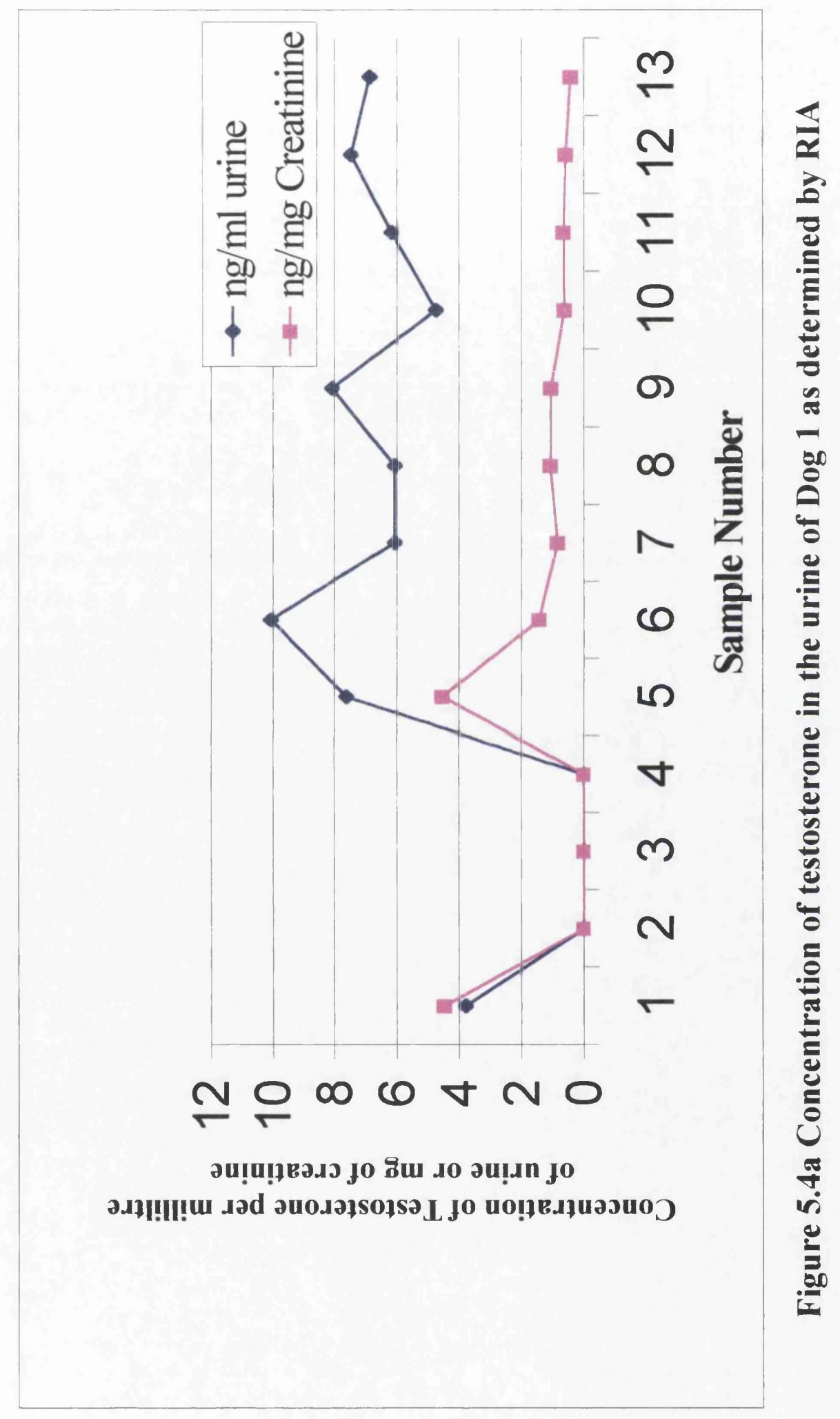




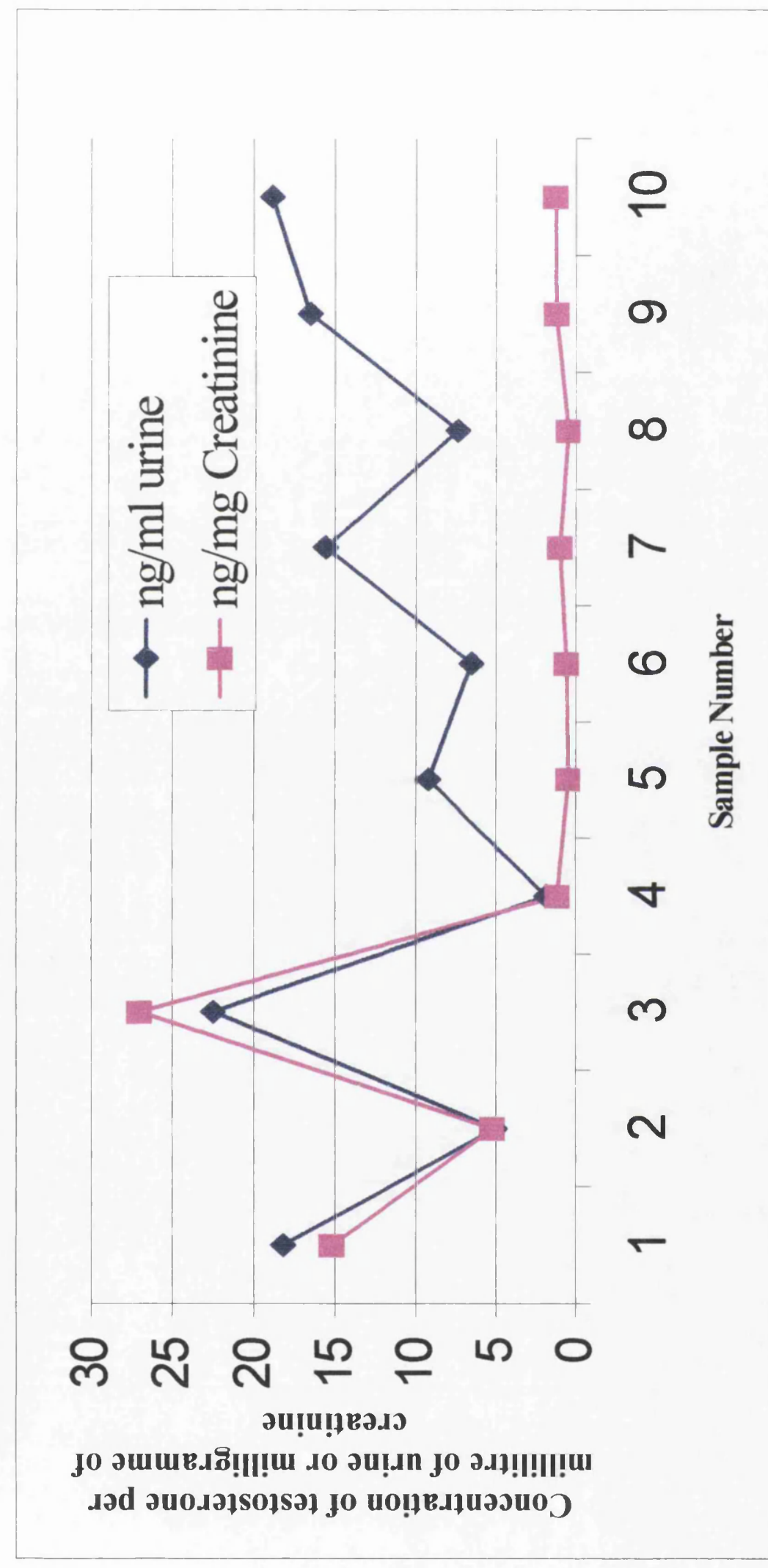

$\frac{1}{2}$

2

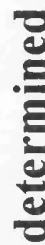

ซ

$\sim$

8

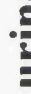

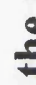




\section{Dosing with Radiolabelled testosterone.}

Preparation of radiolabelled testosterone:

In order to investigate further the metabolism of testosterone in the racing greyhound, it was decided to dose an animal with a very small amount of radiolabelled testosterone of a high specific activity. By injecting this small amount, it was hoped that little disruption to the endogenous pool of steroids and consequently to the steroid metabolism would occur. Details of the experimental protocol are given in chapter 4. It was not possible to use trained greyhounds that were actively involved in racing for these experiments due to the use of the radiolabelled compound.

In addition, the original experimental protocol included the collection of faeces. The greyhound used for this radiolabelled study was effectively a house-trained animal. Even though the greyhound was not enclosed in its own kennel, it refused to defecate in its living environment. After three days, the animal was in such distress that it had to be let out of the cage to defecate. It was decided then to suspend faeces collection for the sake of the health of the greyhound.

\section{Plasma concentrations:}

Due to the very low concentration of testosterone given to the animal (ca.6 nanogrammes), no attempt was made to identify the individual radiolabelled compounds. The level of radioactivity found in the plasma of the greyhound is given in figure 5.5. Using the initial portion of the graph, it is possible to make an estimation of the apparent volume of distribution of testosterone. When extrapolated this is calculated at 5.8 litres. It is known that the weight of the greyhound was 37.5 kilogrammes and that approximately $8 \%$ of the animal's weight is blood volume. Therefor, this greyhound has an approximate blood volume of 3 litres. Consequently, approximately $50 \%$ of the given dose has left 
the blood and entered the tissues. Rather than a steady decline in the level of radioactivity, between 8 and 24 hours the levels rise again. This would indicate that some of the material is either being released back into the body from the tissues, or more likely that enterohepatic re-circulation is occurring and the radiolabelled material is being reabsorbed from the gut.

\section{Levels of Radioactivity in the Urine:}

24-hour total collection of urine was possible in the steel metacages. The results of the urine collection are given in figure 5.6. The results are expressed both as $\%$ dose eliminated in a given 24 hour period and cumulative dose excreted. Approximately $40 \%$ of the total dose is eliminated in the urine within 144 hours post dose. $22 \%$ of the dose is eliminated between 0 and 24 hours, falling to almost $10 \%$ of the dose in the following 24 -hour period. The remaining $8 \%$ is excreted between 48 and 144 hours post dose. This long window of detection suggests that at least some of the radiolabelled material is being retained in the body in the form of testosterone or metabolites for an extended period. This extended period of detection again supports the hypothesis of enterohepatic recirculation of steroids in the racing greyhound.

\section{Metabolism of deutero-labelled testosterone:}

This part of the study was designed to enlarge significantly the amount of testosterone in the plasma of the racing greyhound and to allow an evaluation of the endogenous pool size of testosterone in the greyhound. The protocols for sample extraction and analysis are given in Chapter 4. 


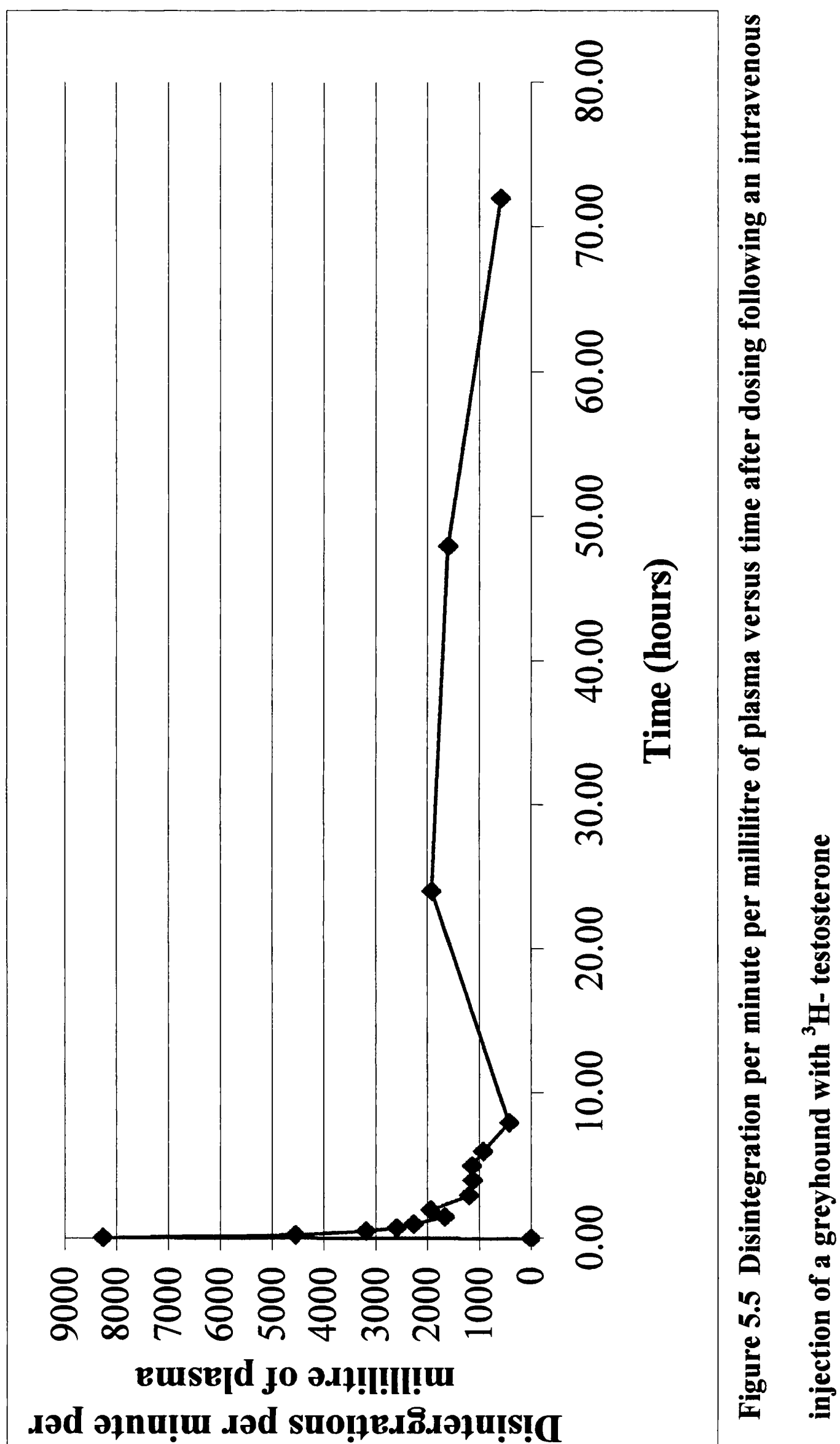




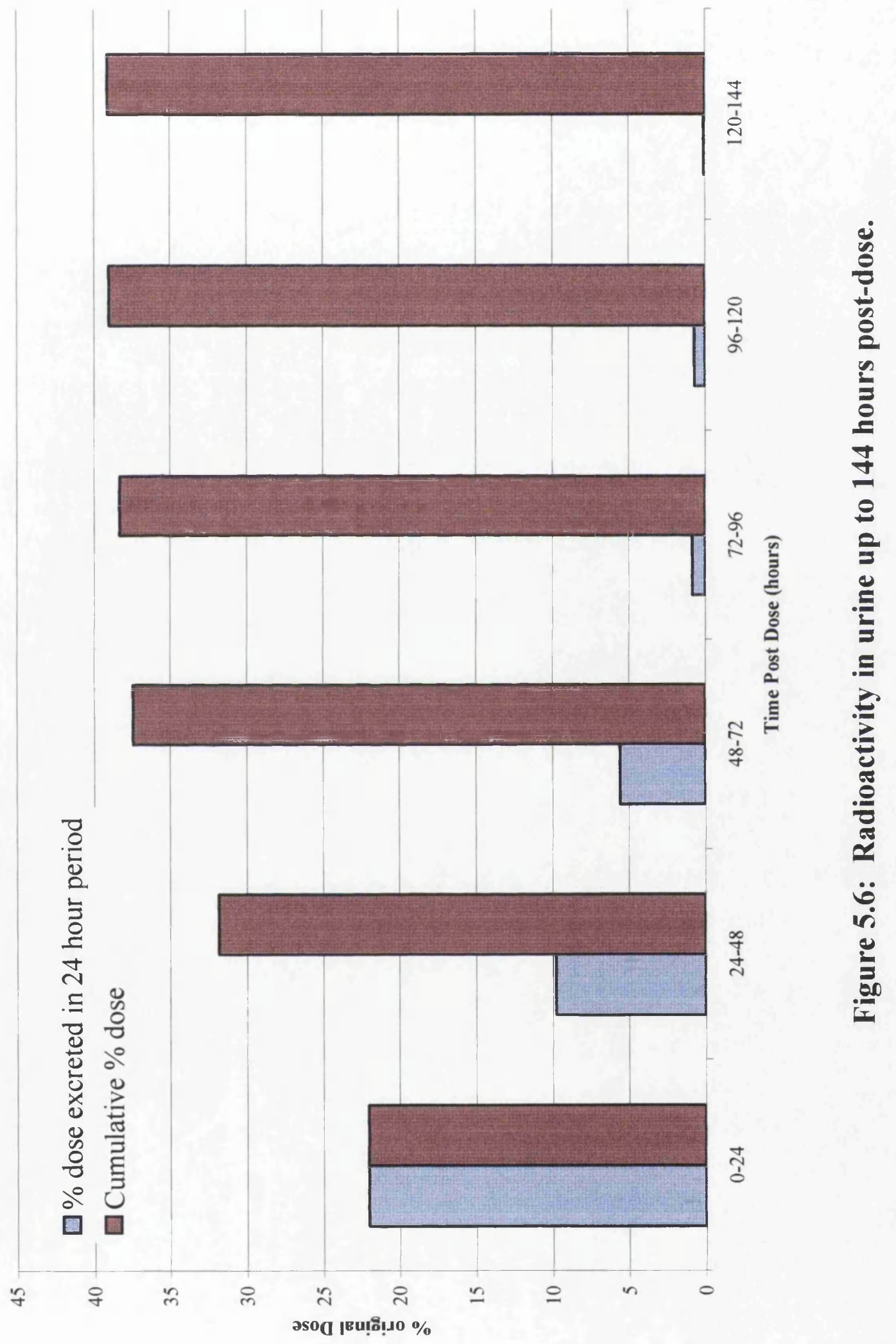


In order to check the recovery of the testosterone from the plasma, plasma samples were spiked with levels of tritiated testosterone and the samples taken through the extraction procedure. The results of the extraction are given in Table 5.4. The mean recovery was $80 \%$, with a standard deviation on the mean of the recoveries of $3.4 \%$ and a coefficient of variation of $4.9 \%{ }^{-1}$.

1.92 milligrammes of the deuterium labelled was given to the greyhound. A graph of the ratio between the labelled and unlabelled testosterone is given in figure 5.7. After 15 minutes, the ratio of labelled material to unlabelled material was 10.5. When the concentration of the deuterium labelled material in the plasma was calculated, this gives an approximate plasma pool size for testosterone in the greyhound of $192 \mu \mathrm{g}$. This is about 6 times the reported value of about $35 \mu \mathrm{g}$ in the human male. The $192 \mu \mathrm{g}$ pool size seems quite large for a 37 kilogramme greyhound. It is possible that in injecting the 2 milligrammes of material, the pool size was disrupted to such an extent that the apparent pool size is inflated.

\section{Conclusions}

There are little or no steroids excreted in the urine of a normal healthy male greyhound. This is consistent with many other species (cows, sheep, goat) but differs from the human. It is possible to determine the presence of testosterone in the urine of the male racing greyhound using radioimmunoassay, but due to the inherent crossreactivity of immunoassays, it is not possible definitively to quantify the testosterone in urine. If the assay is specific enough only to measure testosterone, the levels present are in the order of a few nanogrammes per millilitre of urine. These levels would be too low to definitively identify the 
presence of testosterone by full scan mass spectra with the equipment available. While the use of the labelled forms of testosterone have provided some information regarding the metabolism of testosterone in the male animal, a significant amount of work remains to be done. In the female animal, it was possible to determine a steroidal profile in urine. However, the presence of the steroids may be present due to the onset of oestrus rather than because they are present at all times. 


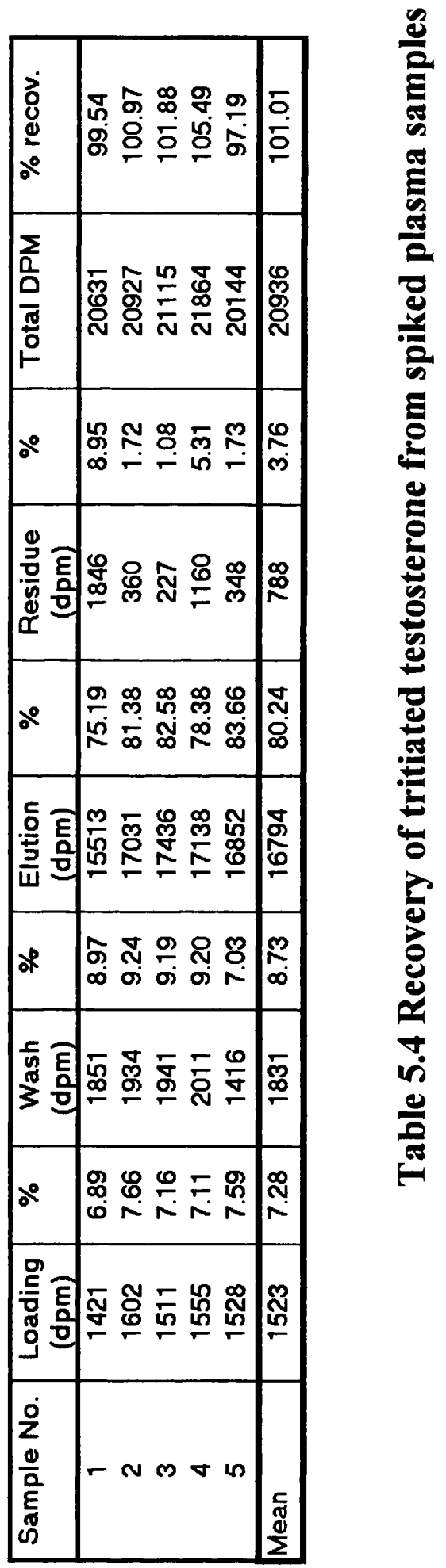




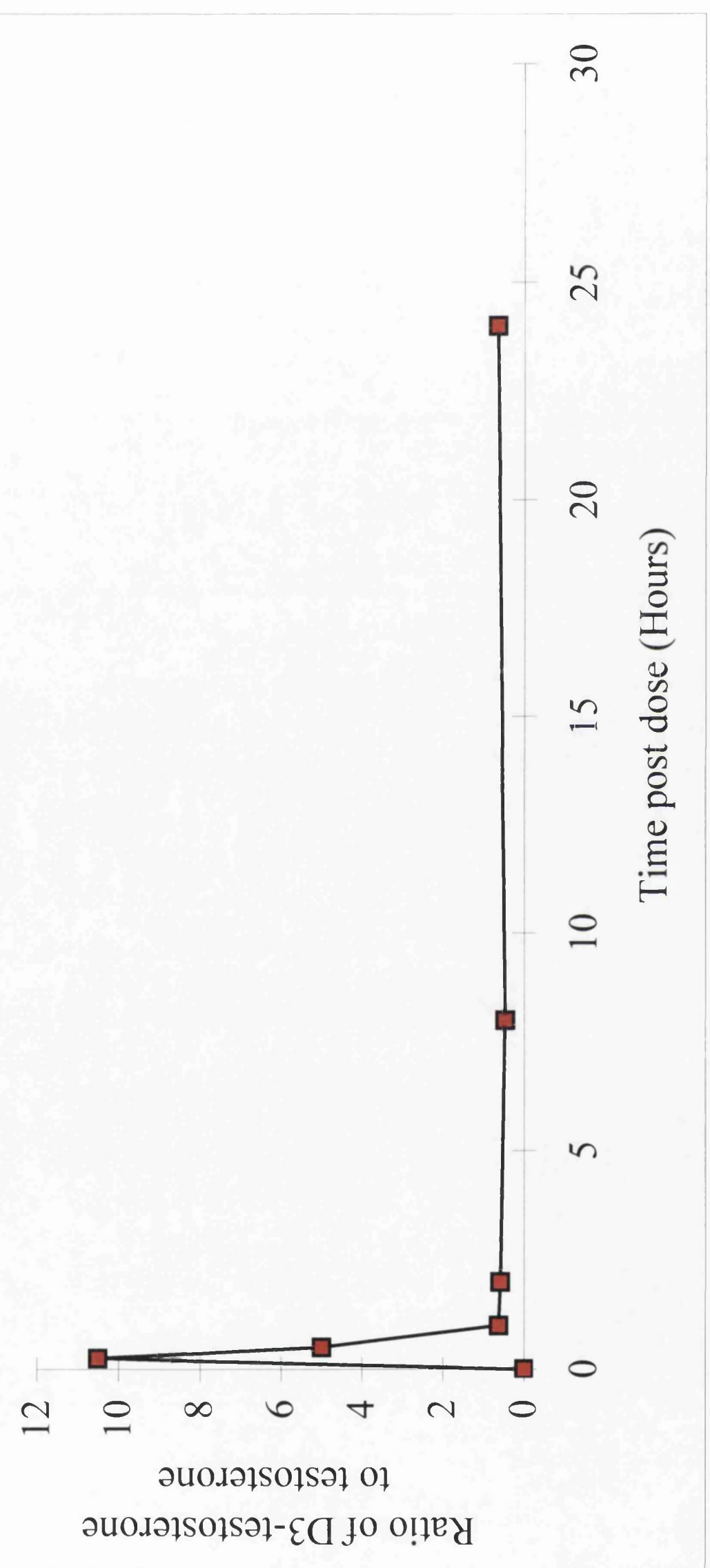

$-$

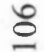

జ

包

을

\&

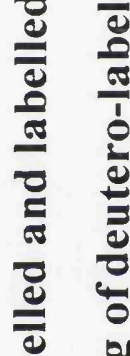

है

를 줄

는 


\section{Chapter 6 Metabolism of Nandrolone and Boldenone}

\section{Introduction}

Nandrolone (also known as 19-nortestosterone or trade name Laurabolin) and boldenone (also known as Dehydrotestosterone or trade name Vebonol) when this work was carried were thought to be two synthetic steroids which should not naturally be present in the urine of the racing greyhound. More recent information has suggested that nandrolone is, in fact, a naturally-occurring hormone in the horse ${ }^{89}$ and male pigs ${ }^{85}$. The structures of nandrolone and boldenone are given in Figure 6.1 along with those of the esters used in veterinary medicine.

The safety data sheet for Nandrolone Laurate (Laurabolin) states that this compound has marked anabolic and anticatabolic action with negligible androgenic or progestagenic activity. It is formulated to provide persistent activity (up to three weeks) following a single injection and is intended for prolonged anabolic therapy ${ }^{86}$. The safety data sheet for boldenone undecylenate (Vebonol) states that Venonol is suitable for supportive treatment of all conditions in which it is desired to reverse weight loss. The compound is designed to stimulate the appetite of debilitated animals ${ }^{87}$.

\section{Dosing of anabolic steroids}

These preparations are normally given to animals in the form of an intramuscular injection. The esters are suspended in a nonpyrogenic oil base and injected into the greyhound in the hindquarters. The oil and esters are slowly absorbed into the body 
and it is recommended that a single intramuscular injection is given once every two weeks. Esterases in the blood then cleave the two sections of the molecule and allow the free steroid to circulate. Using an intramuscular injection avoids first pass metabolism of the liver that would inactivate the steroid. In these studies, the maximum recommended dose was given to two male racing greyhounds. A period of three months post dose was allowed between injections to allow the steroid biochemistry and metabolism of the greyhounds to return to normal. 
<smiles>CC12CCC3C4CCC(=O)C=C4CCC3C1CCC2O</smiles>

Nandrolone (19-nortestosterone)<smiles>CCCCCCCCCCCCCCCCCCC12CCC3C4CCC(=O)C=C4CCC3C1CC2</smiles>

Nandrolone Laurate<smiles>CC12C=CC(=O)C=C1CCC1C2CCC2(C)C(O)CCC12</smiles>

Boldenone (1-dehydrotestosterone)

Figure 6.1 Structures of Nandrolone, Nandrolone Laurate and Boldenone 


\section{Nandrolone.}

The assay procedure for the final determination of nandrolone in plasma and urine samples is given in chapter 4. Prior to this methodology being established, several derivatives were prepared to determine which would be the most suitable for the analyses. During the initial evaluation studies, it was noted that nandrolone and epitestosterone chromatographed very similar retention times. When the mass spectrometer was operated in the selected ion monitoring mode at a resolution of 3000 , the M-15 peak of epitestosterone containing one carbon 13 atom gave a response on the ion chosen to monitor the molecular ion of nandrolone. This is not normally an problem. However, due to the similar chromatographic behaviour of the two compounds, it was possible that there would be a small but possibly significant contribution to the area of the nandrolone peak, giving an overestimation of the concentration of nandrolone in any samples analysed. Therefore a variety of derivatives were formed to try and separate these compounds.

Figures 6.2a,b, and $\mathrm{c}$ and Figures 6.3 show the mass spectra of the various derivatives of nandrolone and Figure 6.4 shows their structures.

Figure 6.2 shows TMS, MO-TMS and ethoxime-TMS derivatives of nandrolone. Most of the ions in Figures $6.2 \mathrm{a}$ and $6.2 \mathrm{~b}$ are the same. These derivatives were formed in a similar manner to the other oxime-TMS derivatives described in Chapter 4. The main differences in the mass spectra of the oxime derivatives occurred in the molecular ions $(\mathrm{M}+)$ and the $\mathrm{M}-90$ fragment ions. These were simply due to the additional methylene group in the ethoxime derivative. The presence of a fragment ion at $\mathrm{m} / \mathrm{z} 344$ is due to the loss of the oxime moiety from the molecule. 
Figure 6.3 shows the reconstructed mass spectrum of the heptafluorobutyrate of nandrolone. This derivative was formed as described in chapter 4 , along with the trifluororacetate and pentafluoropropionate analogues. The only major differences between the spectra of the three compounds was due to homologous series of fragment ions arising from the addition of extra $\mathrm{CF}_{2}$ groups to the steroid molecule. Of these derivatives, only the heptafluorobutyrate gave sufficient base line resolution of the compounds. Consequently, this was the derivative used for the determination of nandrolone in plasma and urine samples from the two male racing greyhounds. In addition to nandrolone, testosterone, epitestosterone, androsterone and etiocholanolone were determined. Of these compounds, only the 3-keto- $\Delta-4$ steroids formed bis-derivatives. Compounds without a double bond in the A-ring (androsterone and etiocholanolone) formed the mono-derivatives. 


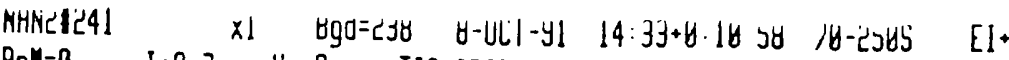

Bph $=8 \quad I=9.7 v \quad H_{m}=8 \quad$ TIC $=6587768080$

NANOROLONE TMS DERIVITIVE

Acnt:

$\begin{array}{ll}G C=256^{\circ} & \text { Sys : KEITH2 } \\ \text { :20CT }\end{array}$

HMR:

63414880

MASS:
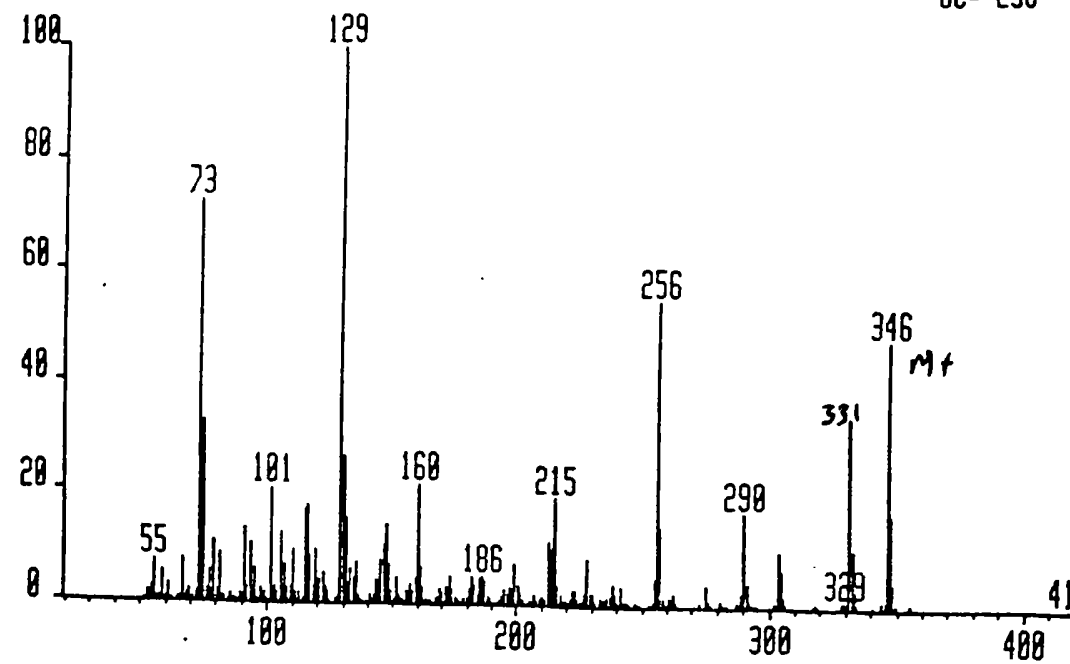

NAM 31255

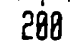

$B p h=8$

$I=6.84 \quad H_{A}=8 \quad$ TIC $=525288888$

MANOROLONE MO-TMS OERIVITIVE

$180 \quad 73$
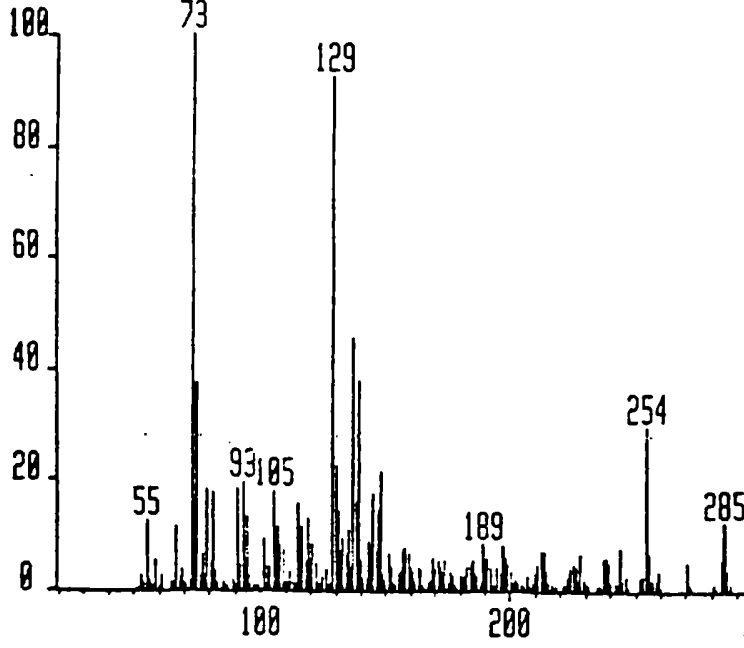

388

NAN4\$277 $x 1 \quad$ Bgd=288 $\quad 8-8 C T-91 \quad 15: 38+8: 11: 51 \quad 78-2585 \quad$ El +

$B p H=8 \quad I=4.7 v \quad H_{A}=8 \quad$ IIC $=2988368808$

NAMOROLOHE ETHOXYAMIHE-THS OERIVITIUE

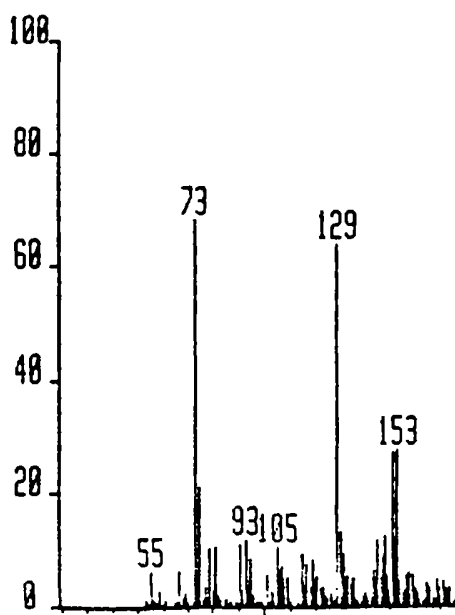

180

288

Acnt

$C C=26 C^{0}$

Sys: KEITH2

Cat : 20CT

Acnt: Sys: KEITH2 HMR: 39837808

$G C=268^{\circ} \quad$ Cal :2OCT

Acnt: Sys: KEITH2 HMR: 39837808

MASS

688

588

\section{5}

389

HIIR: $\quad 38995800$

MASS:

389 


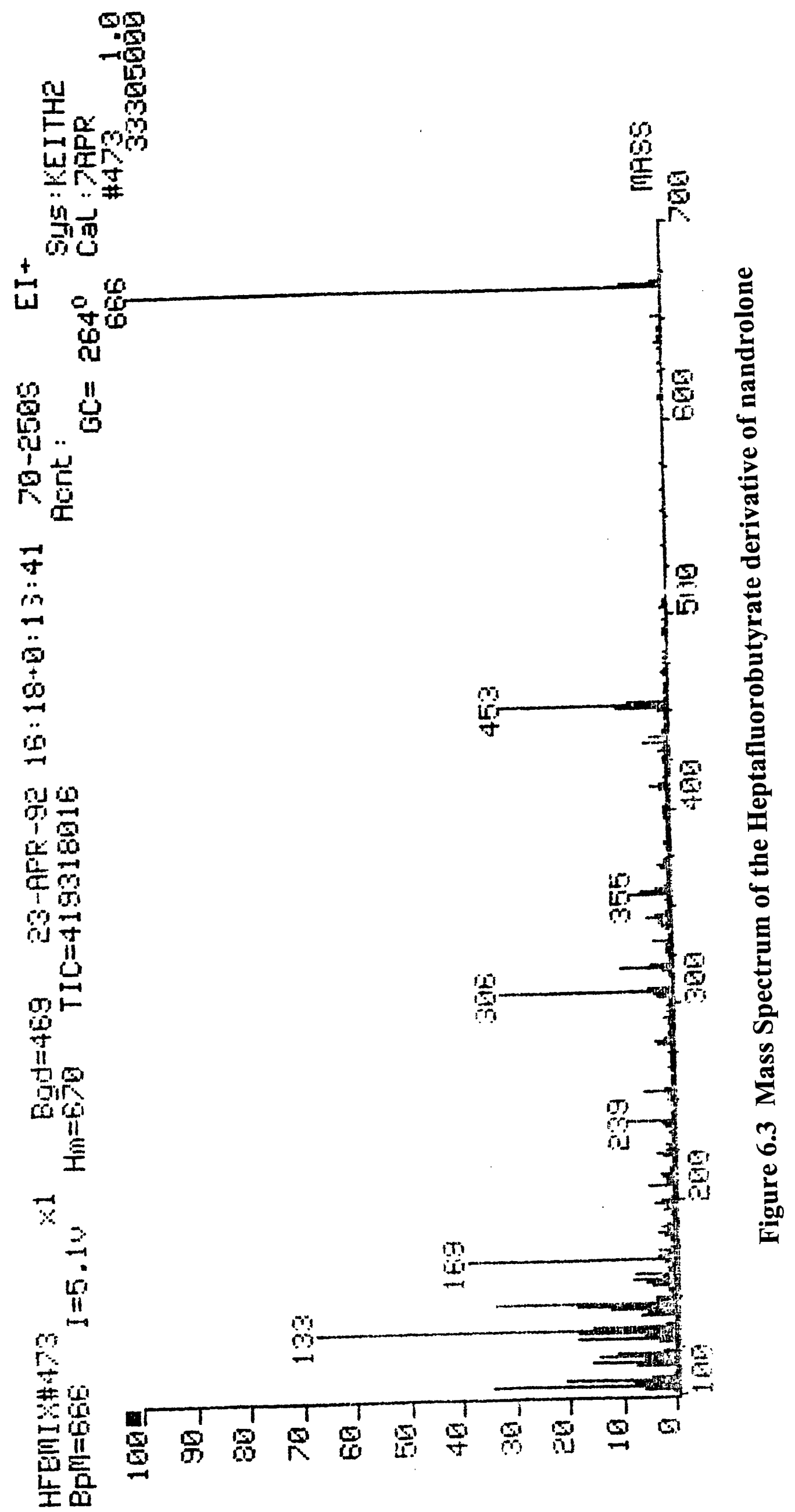


<smiles>CC(C)(C)[SiH2]OC1CCC2C3CCC4=CC(=O)CCC4C3CCC12C</smiles>

Trimethylsilyl of nandrolone<smiles>[R]ON=C1C=C2CCC3C(CCC4(C)C(O[SiH2]C(C)(C)C)CCC34)C2CC1</smiles>

$\mathrm{R}=\mathrm{CH}_{3}$, Methoxyamine derivative $\mathrm{R}=\mathrm{CH}_{2} \mathrm{CH}_{3}$, Ethoxyamine derivative

Oxime-TMS derivatives of Nandrolone<smiles>[R]C(=O)OC1=CCC2C(=C1)CCC1C2CCC2(C)C(OC([R])=O)CCC12</smiles>

$\mathrm{R}=\mathrm{CF}_{3}$, trifluoroacyl derivative $\mathrm{R}=\mathrm{CF}_{2} \mathrm{CF}_{3}$, Pentaftuoroacyl derivative $\mathrm{R}=\mathrm{CF}_{2} \mathrm{CF}_{2} \mathrm{CF}_{3}$, Heptafhoroacyl derivative

Perfluoroacyl derivatives of nandrolone

Figure 6.4 Chemical structures of nandrolone derivatives formed. 


\section{Dosing of the Racing Greyhounds}

A commercial preparation of nandrolone laurate (Laurabolin) was used to dose the greyhounds. The experimental protocol called for the collection of plasma samples predose and then at $+1 \mathrm{~h},+2 \mathrm{~h},+3 \mathrm{~h},+4 \mathrm{~h},+5 \mathrm{~h},+6 \mathrm{~h},+8 \mathrm{~h},+24 \mathrm{~h},+2$ days, +3 days, +7 days, +7 days, +8 days, +9 days and +10 days post dose. Urine samples were collected as and when produced. Complete urine collections were not possible for the reasons stated earlier. Blood samples were collected from the saphenous vein. It was hoped that it would be possible to time-trial the greyhounds at a local greyhound track while they were being affected by the nandrolone injection. However, on the advice of the professional trainer, the animals were not time-trialed as he believed the collection of blood samples would hinder and slow down the greyhounds and might have resulted in injury to the animals.

\section{Dog 1 Dosing record and clinical signs}

This animal weighed 33.5 kilogrammes at dosing and was given 1 millilitre of Laurabolin intramuscularly. This is the equivalent of 50 milligrammes of nandrolone laurate $(1.5 \mathrm{mg} / \mathrm{kg})$ or approximately 30 milligrammes of nandrolone $(0.9$ $\mathrm{mg} / \mathrm{kg}$ ). Blood samples were collected from the greyhound up to 5 hours post dose. No 6 hour sample was collected as it was becoming more and more difficult to collect a blood sample and a decision was made not to stress the animal unduly. It was still difficult to withdraw a blood sample for Dog 1 up to 7 days post dose. After this time sampling became far easier. It is possible that the greyhound had relatively fragile veins which needed time to recover, and a relatively intensive sampling programme involving repetitive venipuncture may have been too 
aggressive. On day 2 of the experiment, the greyhound seemed stiff and uncomfortable and a small weight loss was noted. By three days post dose, the animal had returned to normal. This loss of form is likely to have been caused by the blood sampling rather than the effect of the dosing. As soon as the frequent hourly sampling was reduced, the greyhound's condition returned.

\section{Dog 2 Dosing record and clinical signs}

This animal weighed 30.8 kilogrammes at dosing and was given 1 millilitre of Laurabolin intramuscularly. This is the equivalent of 50 milligrammes of nandrolone laurate $(1.62 \mathrm{mg} / \mathrm{kg})$ or approximately 30 milligrammes of nandrolone $(0.97 \mathrm{mg} / \mathrm{kg})$. Blood samples were collected from the greyhound up to 5 hours post dose. No 6 hour sample was collected as it was becoming more and more difficult to collect a blood sample from this dog also. A similar decision was made not to unduly stress the animal. There were no sample collection problems with dog 2 after the 6 hour sample. Similar weight loss and stiffness symptoms were noted on day 2 of the experiment as for dog 1 . These were gone by day three and the animal's condition returned to normal.

\section{Analysis of plasma samples.}

Methodology for the analysis of the samples is given in Chapter 4.

In addition to the analysis of nandrolone, testosterone, epitestosterone, androsterone, etiocholanolne were determined. These compounds were used as internal markers of androgen metabolism and examined to see if they could be used to predict steroid doping. It has been suggested that determining the ratio of androsterone to etiocholanolone can be used to determine exogenous steroid administration ${ }^{88}$. 
Figures 6.5 and 6.6 show the concentration of nandrolone in the plasma of dogs 1 and 2 respectively. As stated earlier, it was expected that nandrolone was an exogenous steroid to the greyhound. However, nandrolone was detected in the pre dose plasma samples examined. Dog 1 had an endogenous level of approximately $60 \mathrm{ng} / \mathrm{ml}$ while $\operatorname{dog} 2$ had a level of $30 \mathrm{ng} / \mathrm{ml}$. Following the administration of Laurabolin to each dog a similar pattern was observed. The levels of nandrolone rose in the initial few hours post dose but fell below the predose concentration in the plasma. As time passed, plasma levels again rose to a maximum value of $154 \mathrm{ng} / \mathrm{ml}$ and $125 \mathrm{ng} / \mathrm{ml}$ for $\operatorname{dog} 1$ and dog 2 respectively. A steady state concentration did not occur in the plasma and consequently the release of the nandrolone from the injection depot appeared to be episodic.

Figure 6.7 shows the level of testosterone in the plasma of dog 1 following administration of the steroid. In both greyhounds there was a significant drop in the concentration of testosterone in the plasma, which probably reflected suppression of the natural androgen production in the greyhound by the nandrolone.

Figure 6.8 shows the ratio of testosterone to epitestosterone in plasma for dog 1 . This figure shows an apparently good match between the plasma concentration of testosterone and the testosterone:epitestosterone (T:E) ratio for dog 1. The plasma $\mathrm{T}: \mathrm{E}$ ratio is at its lowest when the concentration of nandrolone is greatest in dog 1. The levels of testosterone fell dramatically below the limit of detection for the assay, consequently, it was not possibly to determine if the same pattern existed for $\operatorname{dog} 2$. 


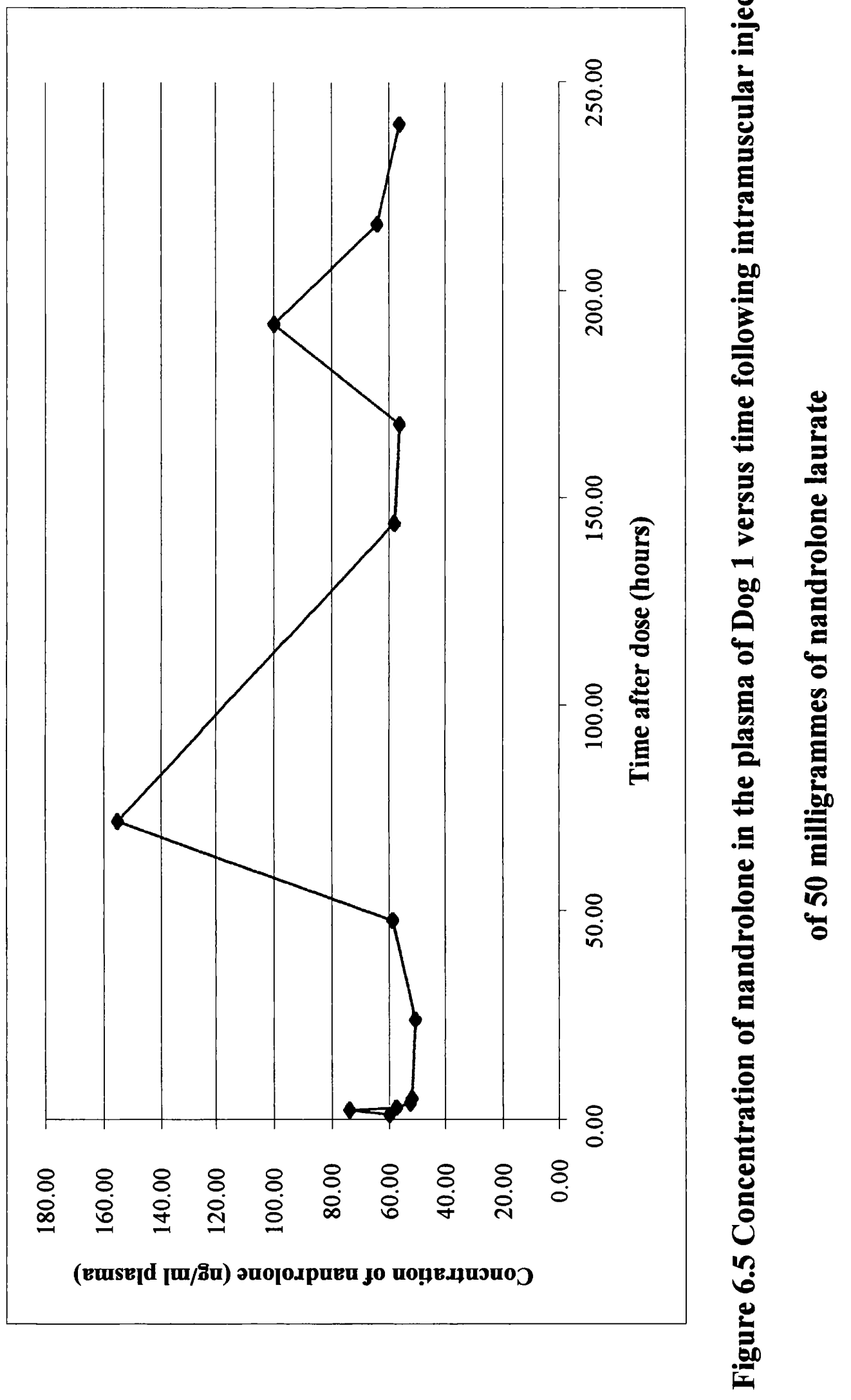



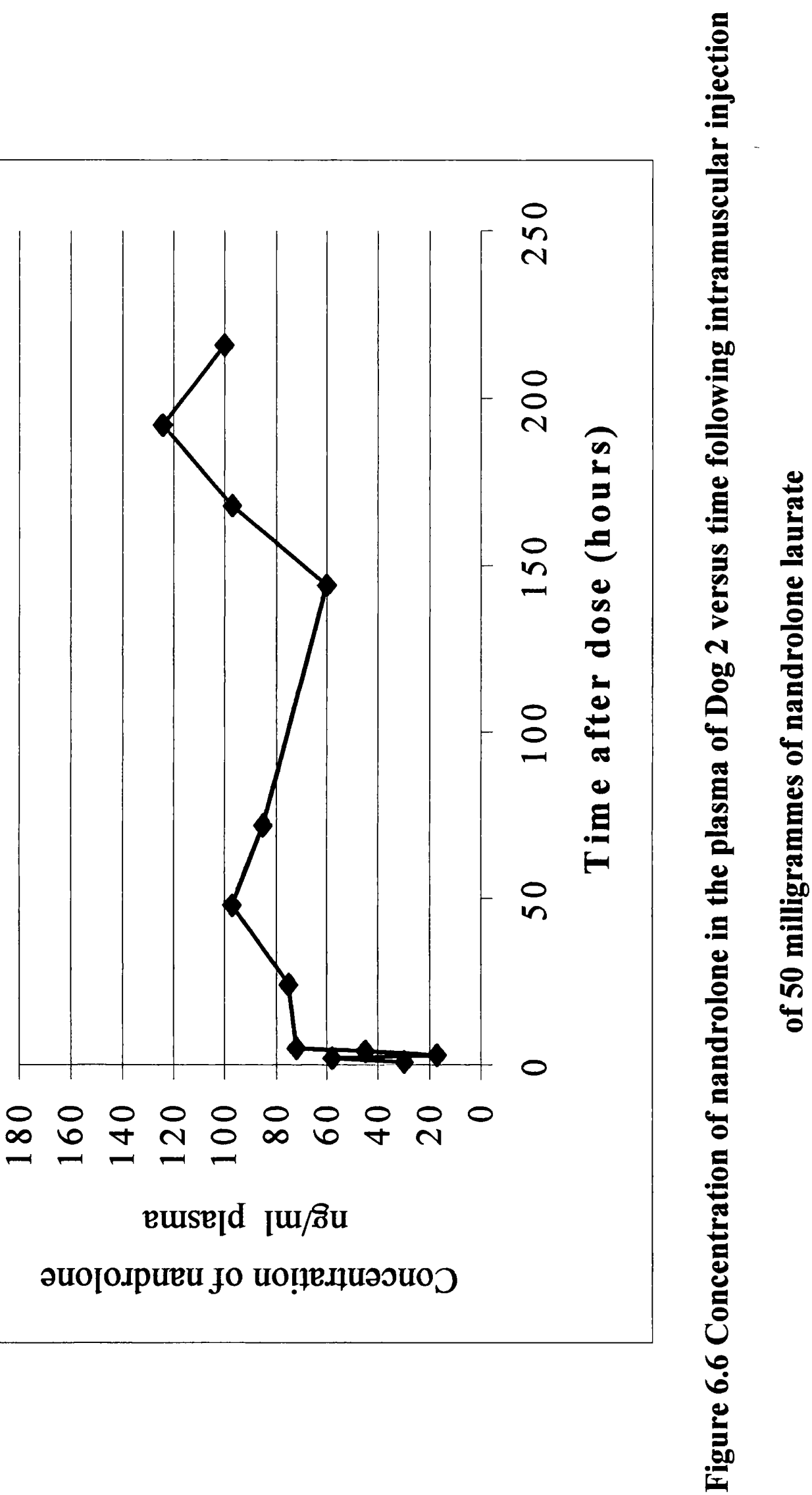

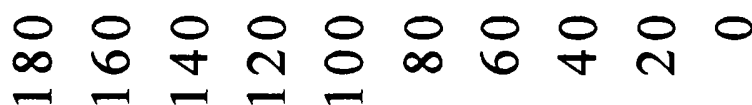

euse [d [u/ôu

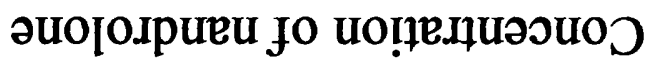


This may provide the basis of a simple screen to determine if exogenous anabolic steroid have been given to a greyhound. Caution must be exercised however as this pattern was not repeated in the other greyhound and it may be an artefact caused by disruption to the natural androgen metabolism by the introduced nandrolone.

Figure 6.9 shows the ratio of androsterone to etiocholanlone for $\operatorname{dog} 2$. As for testosterone and epitestosterone, the concentration of these compounds in plasma fell following the administration of nandrolone. It was not possible to determine a similar graph for $\operatorname{dog} 1$ as the levels of androsterone and etiocholanlone fell below the limits of detection for the instrument. As can be quite clearly seen from the inserted trendline, there does not appear to be any significant change to this ratio in plasma. This ratio in plasma does not appear to be reliable as an indicator of anabolic steroid use in the racing greyhound. In addition, many trainers would probably find it unacceptable to 'injure' a racing greyhound by the collection of a blood or plasma before a race when such an injury may impair its ability to race. Sample collection after a race may be an option, but from the two greyhounds studied there may be some residual effect of more than one sampling is made.

\section{Urinary Excretion of nandrolone}

Figure 6.10 shows the concentration of nandrolone (ng) per milligramme of creatinine and per millilitre of urine in the urine of dog 1 . Nandrolone was present in barely detectable concentrations prior to the dosing of this dog. About 24 hours post dose there was an increase in the concentration of nandrolone (ng) per milligramme of creatinine to 0.39 nanogrammes nandrolone per milligramme creatinine. This fell over the next 10 days back towards the starting levels. A similar pattern existed for the concentration of nandrolone per millilitre of urine. A 
maximum concentration of $6.6 \mathrm{ng} / \mathrm{ml}$ of nandrolone was reached 48 hours post administration.

Figure 6.11 shows the concentration of creatinine in the individual urine specimens over an extended time period. The mean concentration of creatinine is plotted as the dotted line. As can be clearly seen, during the 11 day period after dosing, the levels of creatinine were continually below the average value for dog 1 . This is probably due to the anticatabolic effect of the nandrolone, reducing protein metabolism and increasing the amount of protein retained within the body. Therefore, if a normal creatinine range can be established for dog 1 it would be probable that creatinine measurements could help in the identification of anabolic steroid use. It is questionable, however, if such an extrapolation could be made to other dogs. The greyhounds used in these experiments had free access to water at all times. In order to boost creatinine levels in a dog, all that would be required is to deprive the greyhounds of water for a period of time prior to collection of a urine specimen, therefore artificially raising the creatinine levels. 


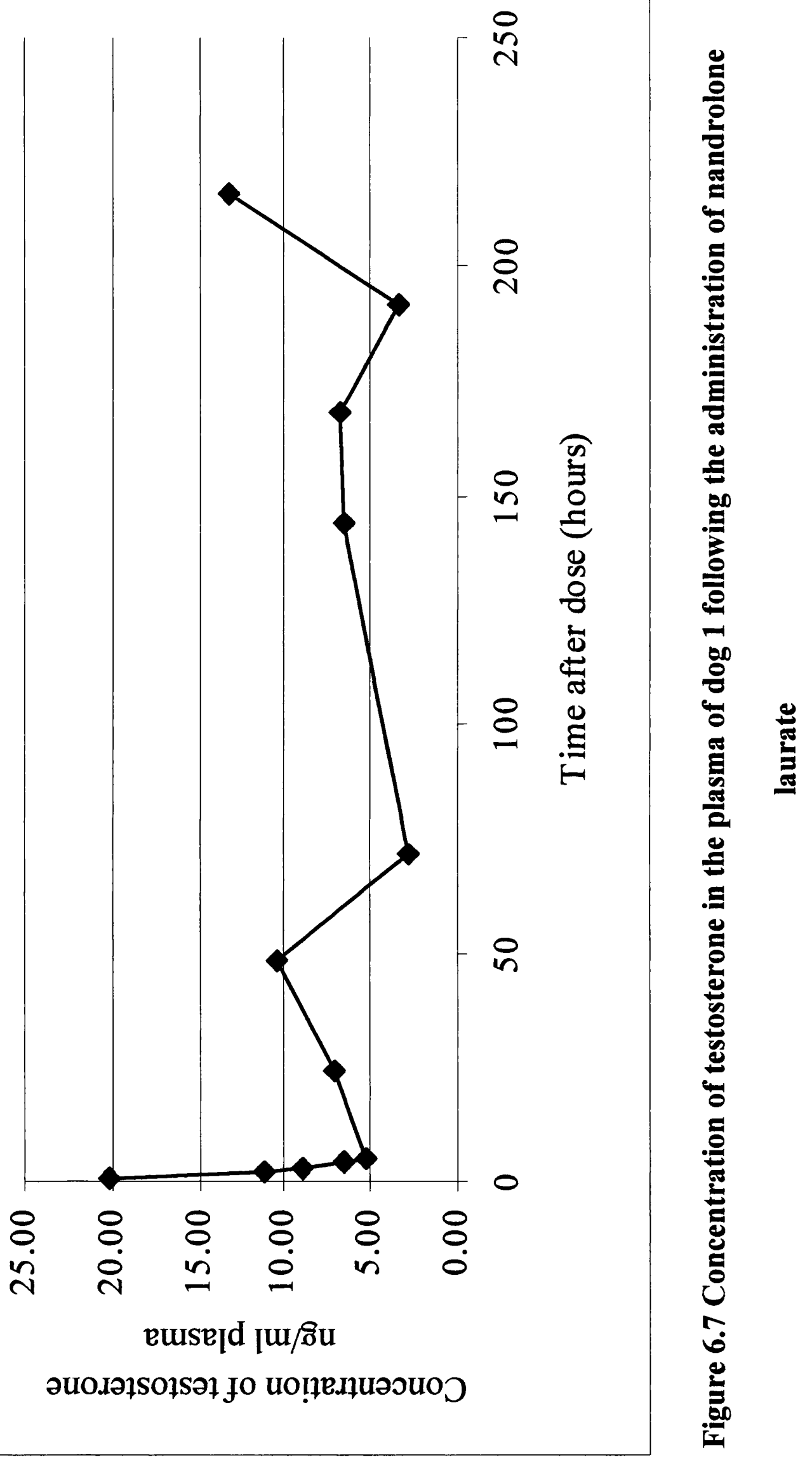



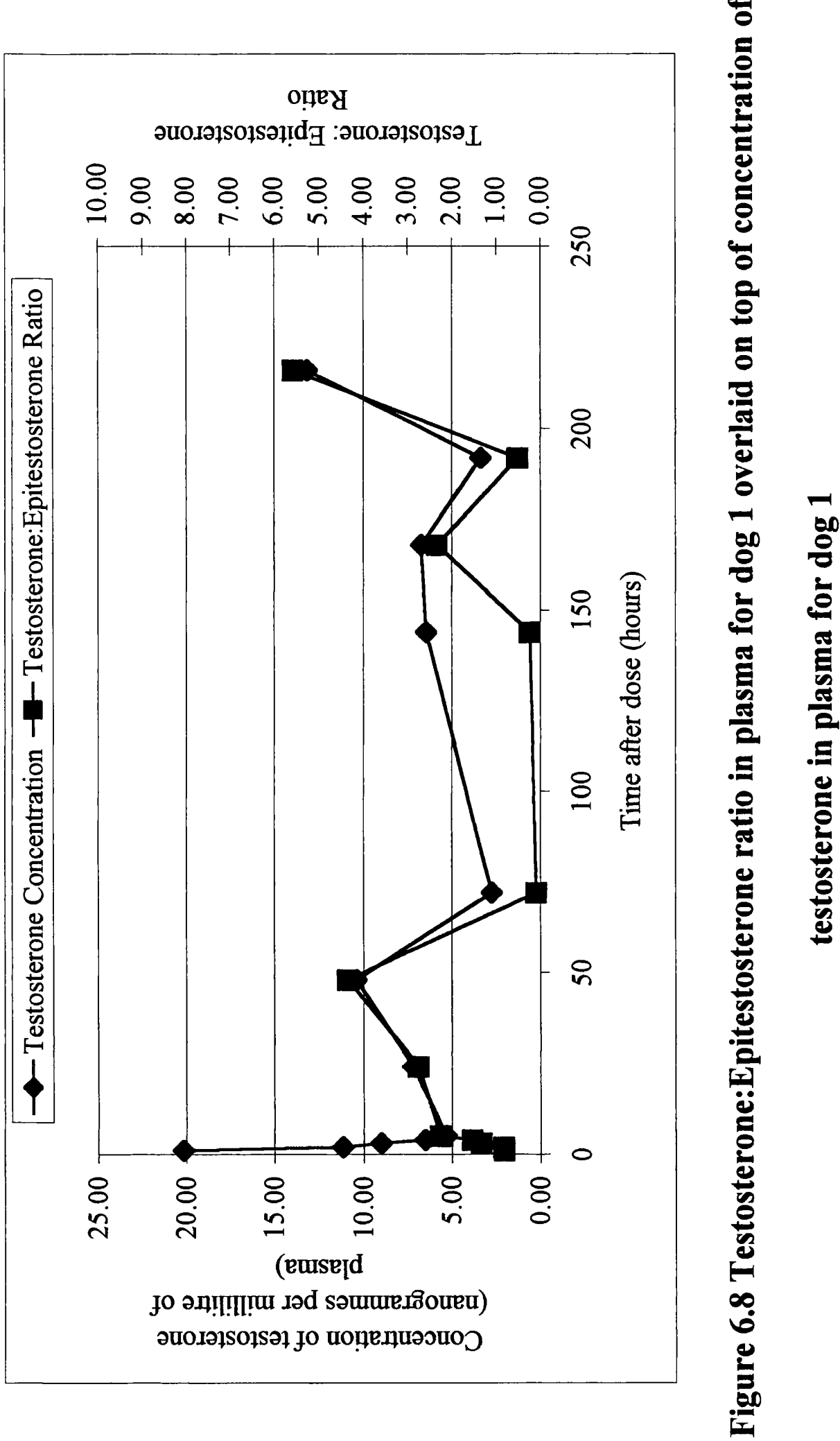


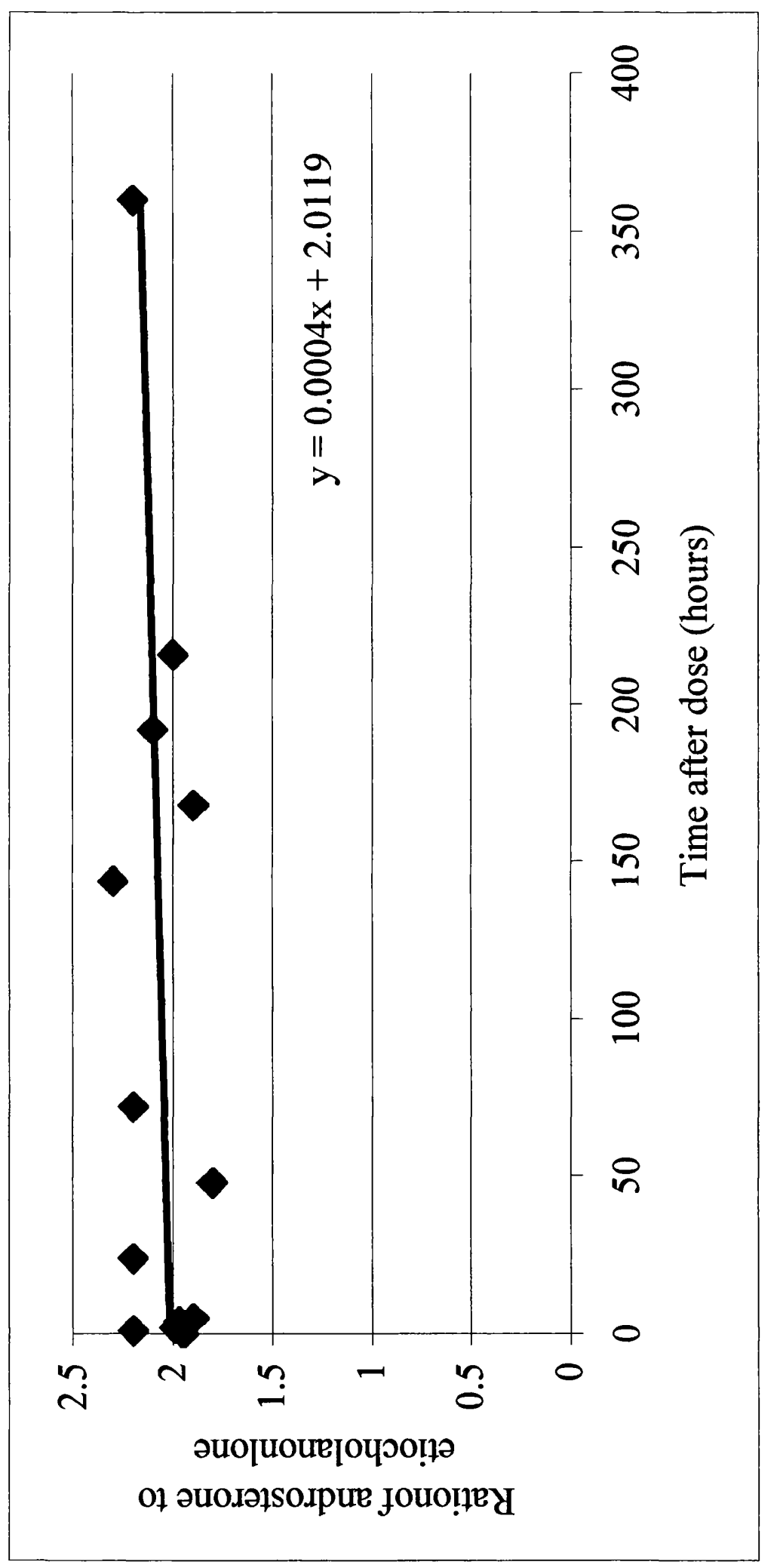

in

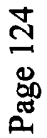

ํㅗㄹ

을

잉

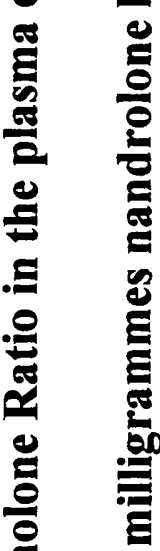

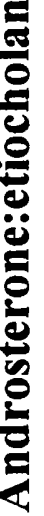

6

를 
The levels of other the steroids monitored were all below the limits of detection in both greyhounds.

As indicated earlier, there is very little data on the metabolism of anabolic steroids in the racing greyhound. The only other publication that deals with the topic in any detail is the previously mentioned publication of Brockwell et $\mathrm{al}^{72}$. These authors gave an upper level of $19 \mathrm{ng} / \mathrm{ml}$ of nandrolone in the urine of a bitch 48 hours post administration and stated that they identified the presence of potentially two metabolites, $5 \alpha$-estran-3 $\beta$-ol-17-one and an unassigned oestradiol. Even when a bitch was given testosterone, the maximum level of testosterone was attained 2 days post administration and was only identifiable for up to three days post administration. The data presented here and that presented by Brockwell and coauthors are consistent with each other.

At the beginning of this study, it was thought that nandrolone was a synthetic steroid. This study along with several others now suggests that nandrolone is, in fact, an endogenous steroid. Its presence has been reported in the colt ${ }^{89}$ and male pigs ${ }^{90}$. Therefor the presence of nandrolone or its metabolites must not be taken as evidence of use unless concentrations are also considered. 


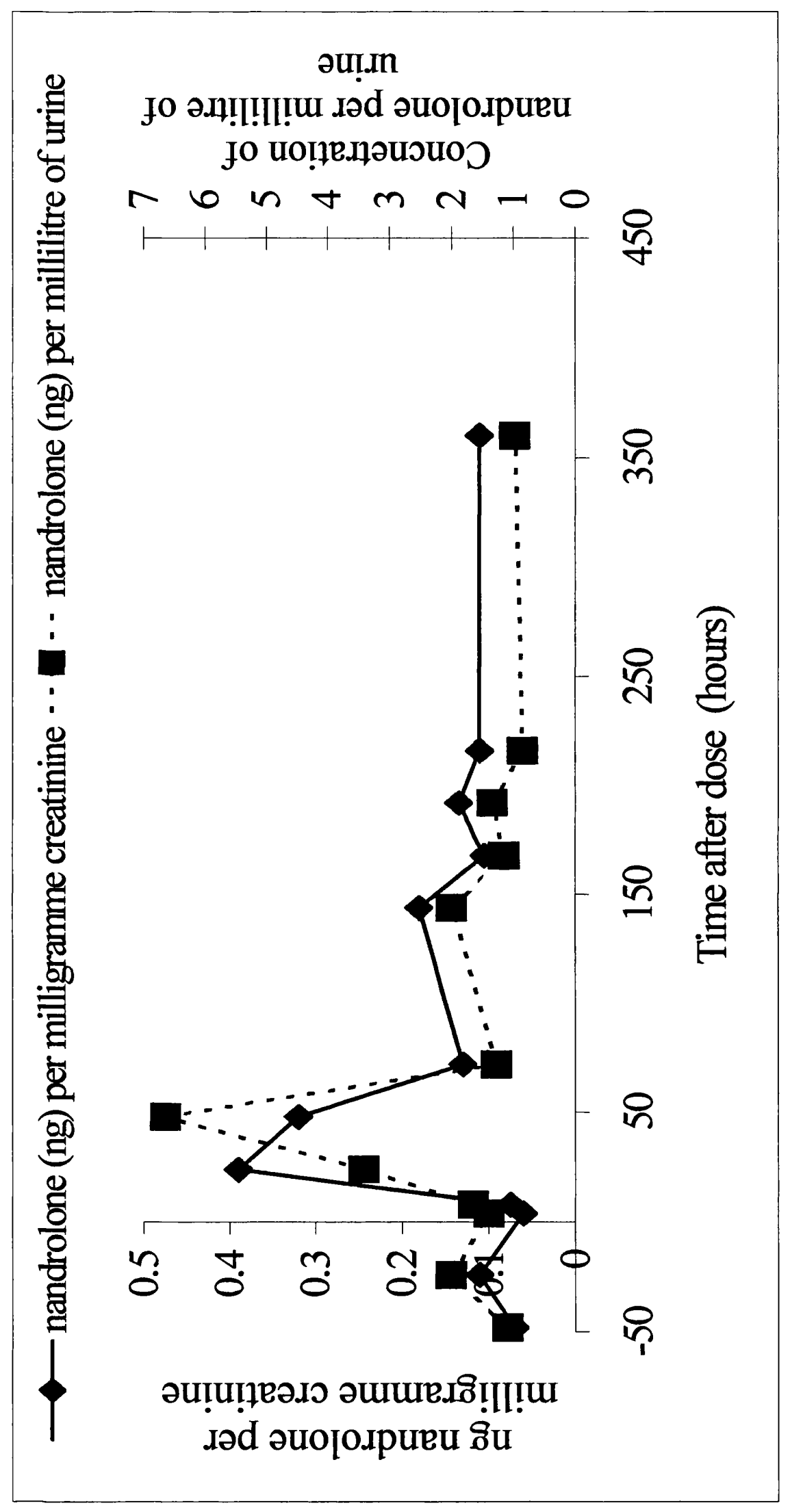

- 


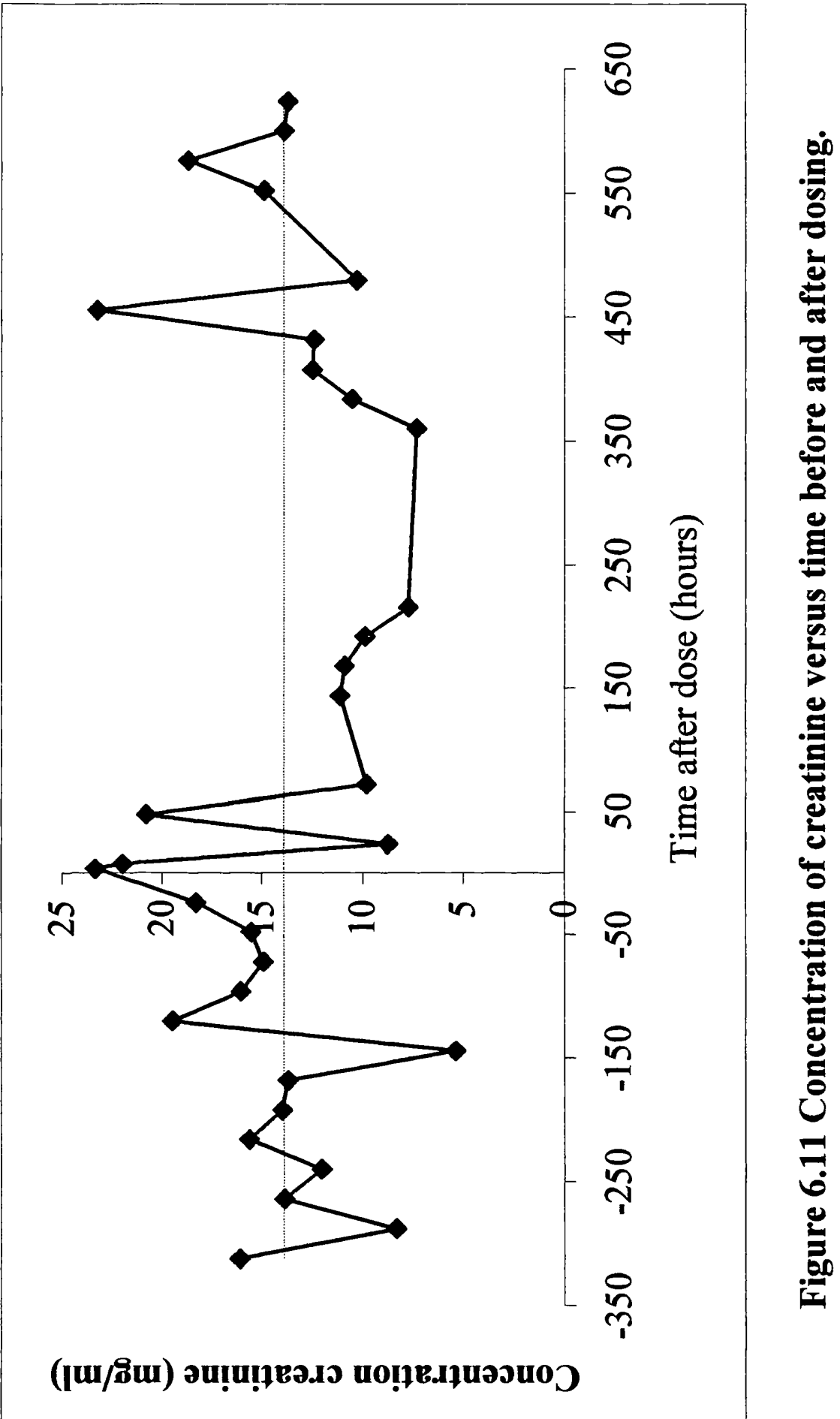




\section{Boldenone}

Boldenone (1-dehydrotestosterone) was administered to two male dogs at a level of 1 millilitre of Vebonol. This is the equivalent of 25 milligrammes of boldenone undecylenate. As for nandrolone, analytical conditions are given in Chapter 4. Samples were collected as described for nandrolone above.

\section{Plasma and Urine Results.}

The presence of boldenone was not detected in any of the urine or plasma samples analysed. This is consistent with the report published by Brockwell et $\mathrm{al}^{72}$. In addition, there did not appear to be the major disruption of the steroid metabolism that occurred with the nandrolone dosing experiment. There are several possible explanations for both the lack of detection of boldenone and the lesser effect on endogenous steroid metabolism.

Significantly less 'free' boldenone was effectively given to the greyhounds than nandrolone. Consequently, both the amount of boldenone available for excretion and the effects on the negative feedback mechanisms when applied to the androgen/steroid metabolism may well be less than those caused by the relatively higher concentration of nandrolone. In addition, it appears that in some species, boldenone is extensively metabolised. Dumasia et al ${ }^{55}$ reported 15 metabolites in the equine castrate, not including isomers. Consequently, depending on the metabolism of the greyhound, it may not be possible to detect any metabolites as each individual metabolite may be present at low concentrations. 


\section{Conclusions.}

It is possible to detect the presence of nandrolone in both the plasma and urine of racing greyhounds following intramuscular administration of this anabolic steroid as its laurate ester. Nandrolone has been shown to be an endogenous steroid in the greyhound. It appears that detection of administered steroids is dependent on both the steroid and the amount given. There does appear to be a suppression of the endogenous steroid concentration, as would be expected, and there may be a relationship between the plasma testosterone:epitestosterone ratio and the plasma testosterone concentration. The levels of creatinine appear to be depressed below the average shortly after dosing and this may provide a detection mechanism for a given animal. 


\section{Chapter 7 The analysis of clenbuterol in the plasma and urine of the racing greyhound.}

\section{Introduction}

Structures of clenbuterol and salbutamol are given in Figure 7.1

\section{Mass Spectrometry Studies}

Prior to extraction and analysis of the biological samples, it was necessary to determine sensitive, specific reliable methodology for the analysis of clenbuterol. A variety of derivatives were formed and the alternative mass spectrometry techniques available were evaluated to determine their suitability.

\section{Derivative Formation 1: Trimethylsilyl derivatives}

Standard solutions of clenbuterol and salbutamol were taken to dryness under a stream of nitrogen at $60^{\circ} \mathrm{C}$. BSTFA containing $1 \%$ TMCS $(0.050 \mathrm{ml})$ was added to each dried residue and sealed in a glass vial. Samples were incubated at $80^{\circ} \mathrm{C}$ for 30 minutes prior to analysis. Portions $(1 \mu \mathrm{l})$ of each sample were analysed by GC-MS system 1.

\section{Derivative Formation 2: Perfluoroacyl derivatives}

Standard solutions of clenbuterol and salbutamol were dispensed into glass vials and taken to dryness under a steam of nitrogen at $60^{\circ}$. Trifluoracetic anhydride, pentafluoropropionic anhydride or heptafluorobutyric anhydride $(0.050 \mathrm{ml})$ was added to form trifluoroacetyl (TFA), pentafluoropropionyl (PFP) and heptafluorobutyryl (HFB) derivatives respectively. The samples were allowed to stand at room temperature for at least 20 minutes prior to analysis. Portions $(1 \mu 1)$ of each sample were analysed by GC-MS using system 1 . 
<smiles>CC(C)(C)NCC(O)c1cc(Cl)c(N)c(Cl)c1</smiles>

Clenbuterol<smiles>CC(C)(C)NCC(O)c1ccc(O)c(CO)c1</smiles>

Salbutamol

Figure 7.1 Structures of Clenbuterol and Salbutamol 


\section{GC MS Conditions}

All analyses were carried out using GC-MS system 1 (as described previously in Chapter 4 ) with the following differences. A DB-5-MS Column (20m x $0.18 \mathrm{~mm}$, df $0.18 \mu \mathrm{m}$ ) was employed in the gas chromatograph. The reagent gas for the chemical ionisation, both positive and negative was ammonia (supplied by BOC, UK). For all full scan studies, the resolution of the mass spectrometer was set to 1000 . For selected ion recording studies, the resolution of the instrument was set to 3000 .

\section{Trimethylsilyl derivative of clenbuterol.}

The mass spectra of the trimethylsilyl (TMS) derivatives of clenbuterol and salbutamol are illustrated in Figure 7.2. The TMS derivative is formed by the action of the derivatisation reagent on the hydroxyl group to form only a mono-TMS derivative. Other authors have employed more rigorous derivatisation conditions $\left(100^{\circ} \mathrm{C}\right.$ for 16 hours $)$ and have formed bis-derivatives of clenbuterol ${ }^{91}$. The second TMS residue attaches to the primary amine group. The base peak of the mass spectrum is at $\mathrm{m} / \mathrm{z} 86$ and corresponds to the cleavage of the side chain of the molecule $-\left\{\mathrm{CH} 2 . \mathrm{NH} . \mathrm{C}(\mathrm{CH} 3)_{3}\right]$. The next most intense peaks are $\mathrm{m} / \mathrm{z} 73$ and $\mathrm{m} / \mathrm{z} 57$, corresponding to cleavage of the TMS group and the tertiary butyl group, respectively. A molecular ion couplet does not appear to be present at $\mathrm{m} / \mathrm{z} 348 / 350$ and the highest mass visible is at $\mathrm{m} / \mathrm{z} 333 / 335$, corresponding to the loss of a methyl group from the parent compound. Of the higher molecular mass peaks, those at m/z 262/264 (M-86) and m/z 243/245 (M105) are the most intense. The former masses correspond to the loss of the side chain already detailed and the latter can be attributed to the loss of the -OTMS side chain plus a methyl group. It is well accepted that ions below m/z 100 are not particularly diagnostic. With the prevalence of these low mass ions in the mass spectrum of clenbuterol TMS derivative, it was decided to investigate alternative derivatives and mass spectrometric techniques. 


\section{Perfluoroacyl derivatives.}

Several other derivatives have been proposed for the analysis of Clenbuterol. Dumasia and Houghtan formed cyclic dimethylsilylmethylene derivatives and employed electron impact ionisation to look at a variety of $\beta$ agonists, $\beta$-antagonists and their metabolites in the urine of horses ${ }^{9}$. Girault et al formed acid anhydride derivatives and employed mass spectrometry and negative ion chemical ionisation to determine the level of clenbuterol at femtomole sensitivity in the plasma of humans ${ }^{8}$. Due to the lack of information on the pharmacokinetics of $\beta$-agonists in the racing greyhound, and with the previous experience of the low levels of steroids in both urine and plasma of racing greyhounds, it was considered appropriate to maximise the sensitivity of the analyses and it was decided to use perfluoroacyl derivatives in combination with negative ion chemical ionisation (NI-CI) mass spectrometry.

As indicated above, Girault et al $^{8}$ formed perfluoroacyl derivatives and used pentafluorpropionic anhydride to form pentafluoropropionyl (PFP) derivatives. The authors justified the use of these derivatives, NI-CI and the extensive time commitment required by stating that the very low doses of clenbuterol given therapeutically (approximately $40 \mu \mathrm{g} /$ day) and the high volume of distribution of clenbuterol (approximately 350 litres) resulted in very low plasma concentrations. Following incubation at room temperature, they proposed the formation of a bis derivative, with rapid degradation to a mono derivative and an accompanying dehydroacylation within the clenbuterol molecule. The reaction scheme is summarised in Figure 7.3. They stated that it was possible to form a bis-derivative by incubation of the reaction mixture at higher temperatures, but a mixture of mono- and bis-derivatives was formed and the ratio between these two derivatives was not consistent 
<smiles>CC(C)(C)NCC(O)c1cc(Cl)c(N)c(Cl)c1</smiles><smiles>CC(C)(C)N(C(=O)C(F)(F)F)C(OC(=O)C(F)(F)F)C(c1cc(Cl)c(N)c(Cl)c1)C(F)(F)F</smiles><smiles>CC(C)(C)N(C=Cc1cc(Cl)c(N)c(Cl)c1)C(=O)C(F)(F)F</smiles>

Figure 7.2 Derivatisation of Clenbuterol as Proposed by Girault et al ${ }^{100}$. 
<smiles>CC(C)(C)NCC(O)c1cc(Cl)c(N)c(Cl)c1</smiles>

$$
\downarrow
$$<smiles>[R]C(=O)Nc1c(Cl)cc(C(OC([R])=O)C([2H])N(C([R])=O)C(C)(C)C)cc1Cl</smiles>

$$
\downarrow
$$<smiles>[R]C(=O)NC=Cc1cc(Cl)c(N([R])C([R])=O)c(Cl)c1</smiles>

Where $\mathrm{R}=-\mathrm{CF}_{3},-\mathrm{CF}_{2} \mathrm{CF}_{3}$, or $-\mathrm{CF}_{2} \mathrm{CF}_{2} \mathrm{CF}_{3}$

Figure 7.3 Proposed Derivatisation reaction of Clenbuterol in this study 
In the present study, initial attempts to form and identify the mono- derivatives were unsuccessful. In all the analyses carried out using any of the perfluoroacyl anhydrides, tris-derivatives were invariably formed and these were accompanied by the dehydration proposed by the previous authors to give a bis-derivative. It was not possible to show the presence of a mono-derivative. This reaction scheme is illustrated in Figure 7.3.

Figures 7.5a, b, and c show the reconstructed mass spectra of TFA, PFP and HFB derivatives of clenbuterol respectively under positive ion electron impact conditions. Differences in the fragmentation patterns for each derivative are accounted for by the different lengths of the side chains of the derivatives.

Figure 7.6 illustrates the positive ion chemical ionisation mass spectra of the TFA and HFB derivatives. The HFB derivative has a base peak of $651(\mathrm{M}+1)$ and a higher mass at $\mathrm{m} / \mathrm{z} 668(\mathrm{M}+18)$, corresponding to the addition of $\mathrm{NH}_{4}{ }^{+}$. The TFA derivative also exhibits the $(\mathrm{M}+1)$ fragment at $\mathrm{m} / \mathrm{z} 451$ but there was no indication of a comparable $(M+18)$ fragment at $\mathrm{m} / \mathrm{z} 468$. In addition, considerable fragmentation exists for the TFA derivative compared to the HFB derivative, resulting in the presence of a large number of diagnostically poor lower masses.

Figures 7.7a, b, and c show respectively the reconstructed mass spectra of TFA, PFP and HFB derivatives of clenbuterol under NI-CI conditions. There are both similarities and differences between the three derivatives. Differences between the spectra of the PFP and HFB derivatives can be explained by the differences in acyl groups attached to clenbuterol by derivatisation. A pseudomolecular ion at (M-1) caused by proton abstraction by the reagent gas exists for both compounds along with the loss of a fluorine atom (M-19) and hydrogen chloride (M-36). The base peaks of both derivatives correspond to a loss of the tertiary butyl group and hydrogen fluoride (M-77). The second most intense peaks correspond to the loss of the previously mentioned groups and $\mathrm{HCl}$ (M-113). The TFA derivative, however, displays neither a 


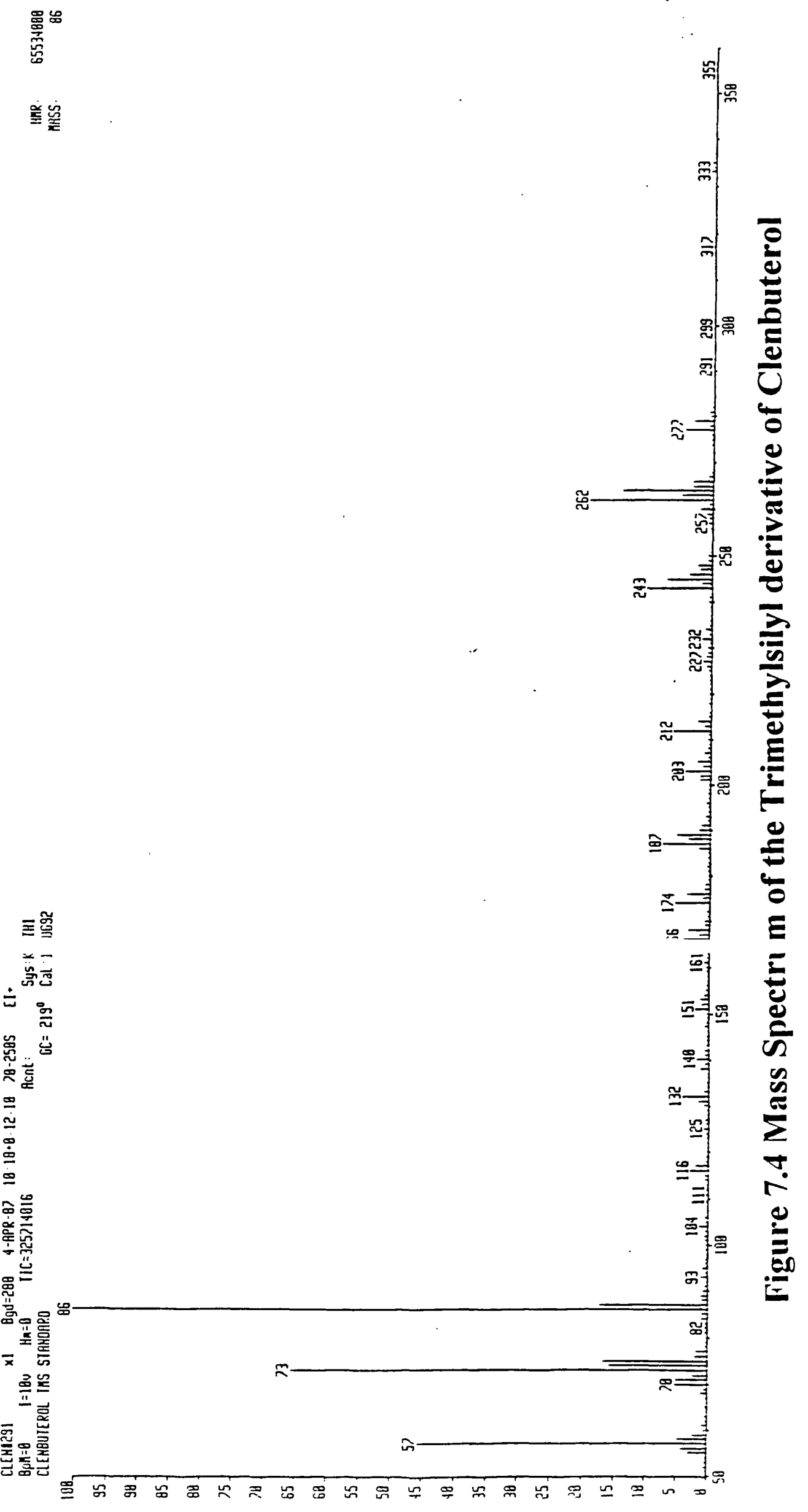




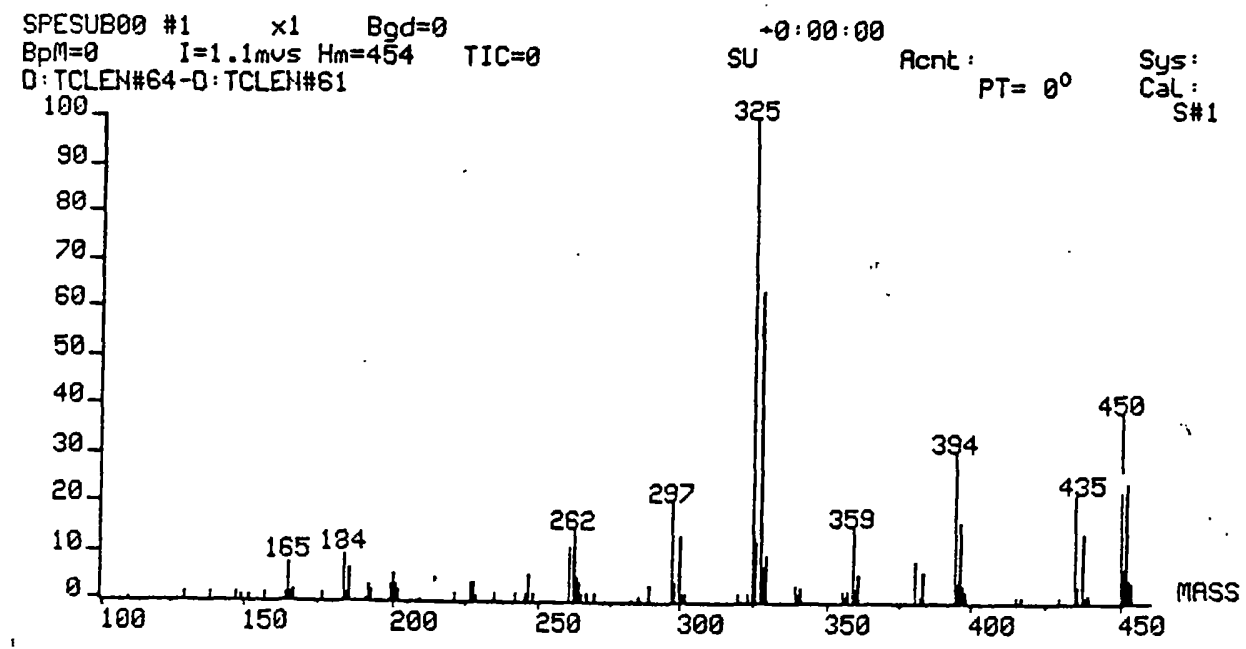

Figure 7.5a Positive Ion Electron Impact Mass spectrum of the TFA derivative of Clenbuterol

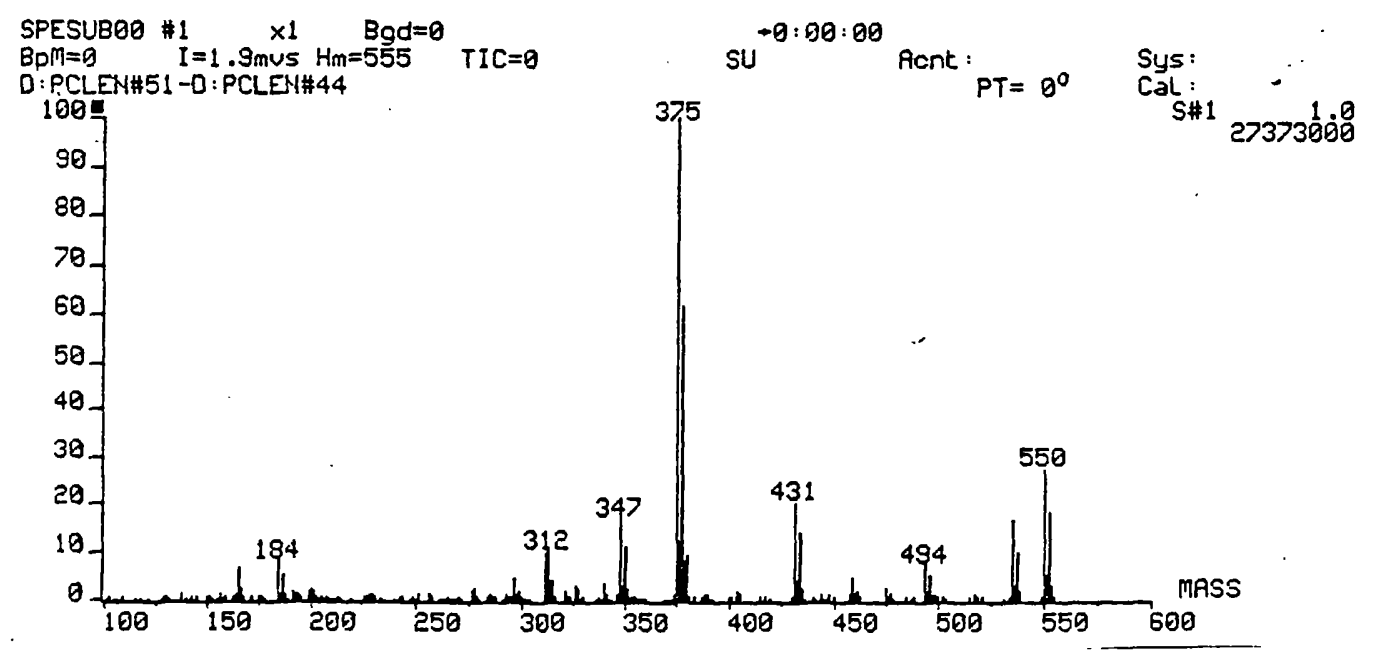

Figure 7.5b Positive Ion Electron Impact Mass spectrum of the PFP derivative of Clenbuterol

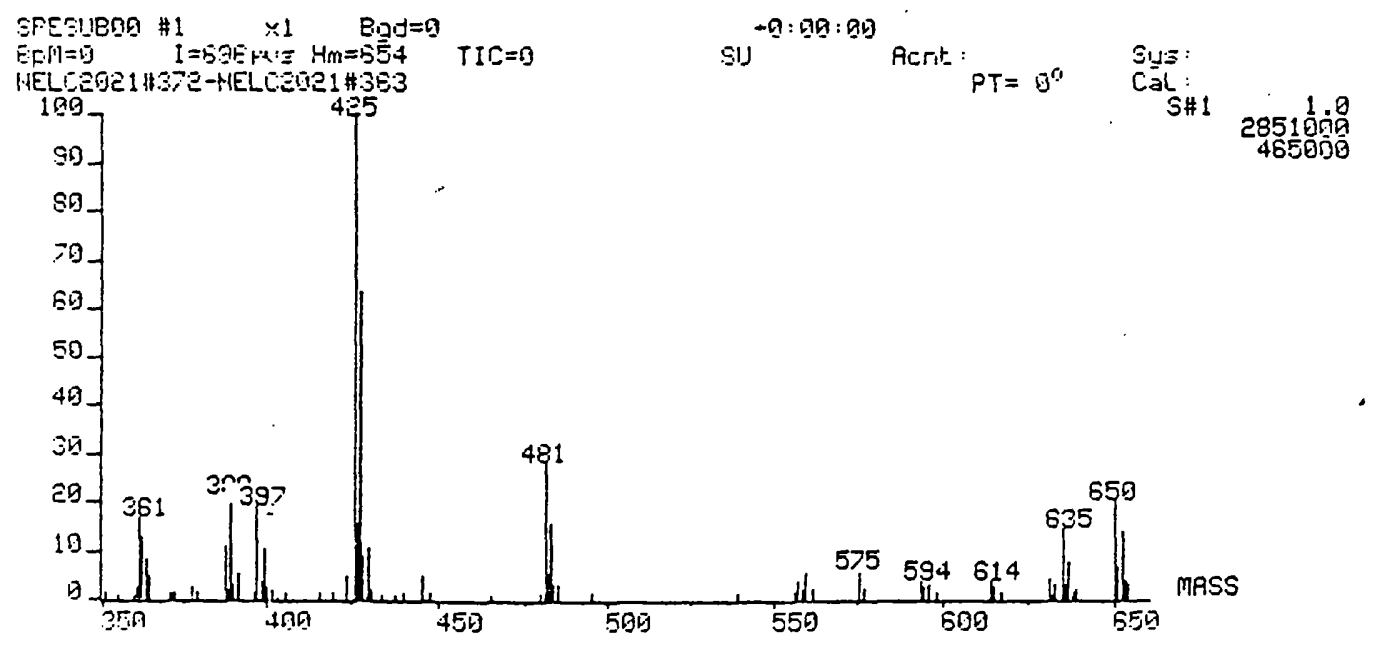

Figure 7.5c Positive Ion Electron Impact Mass spectrum of the HFB derivative of Clenbuterol 


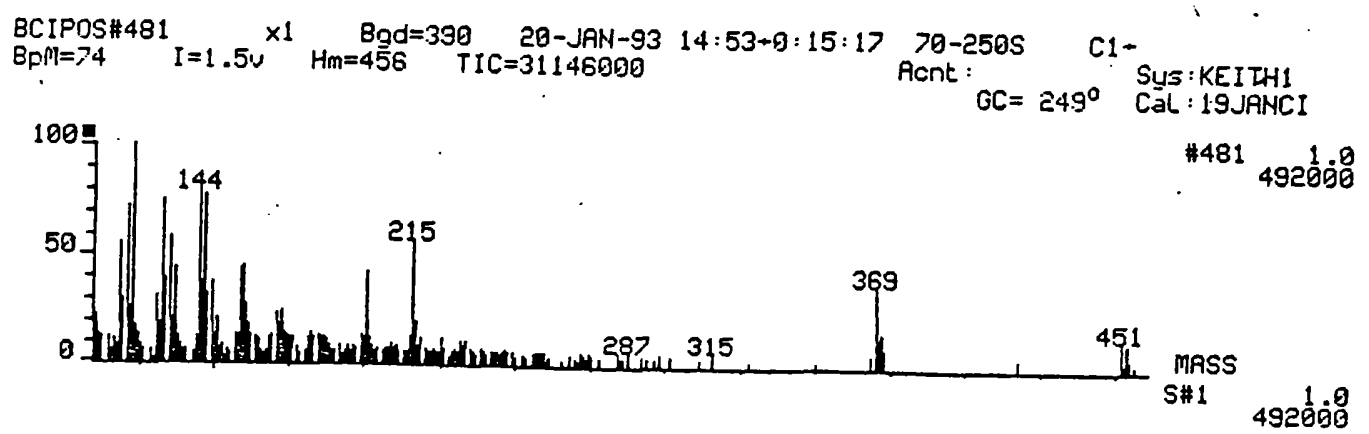

Figure 7.6a Positive Ion Chemical Ionisation Mass spectrum of the TFA derivative of Clenbuterol

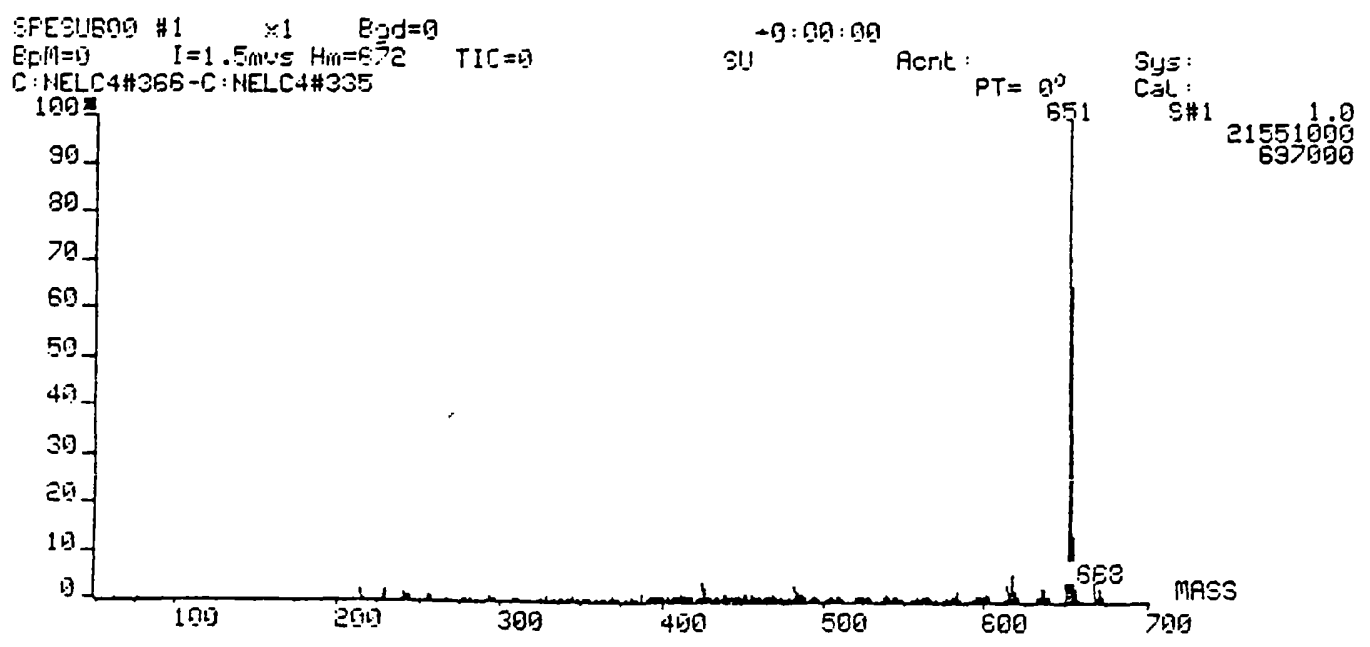

Figure 7.6b Positive Ion Chemical Ionisation Mass spectrum of the HFB derivative of Clenbuterol 


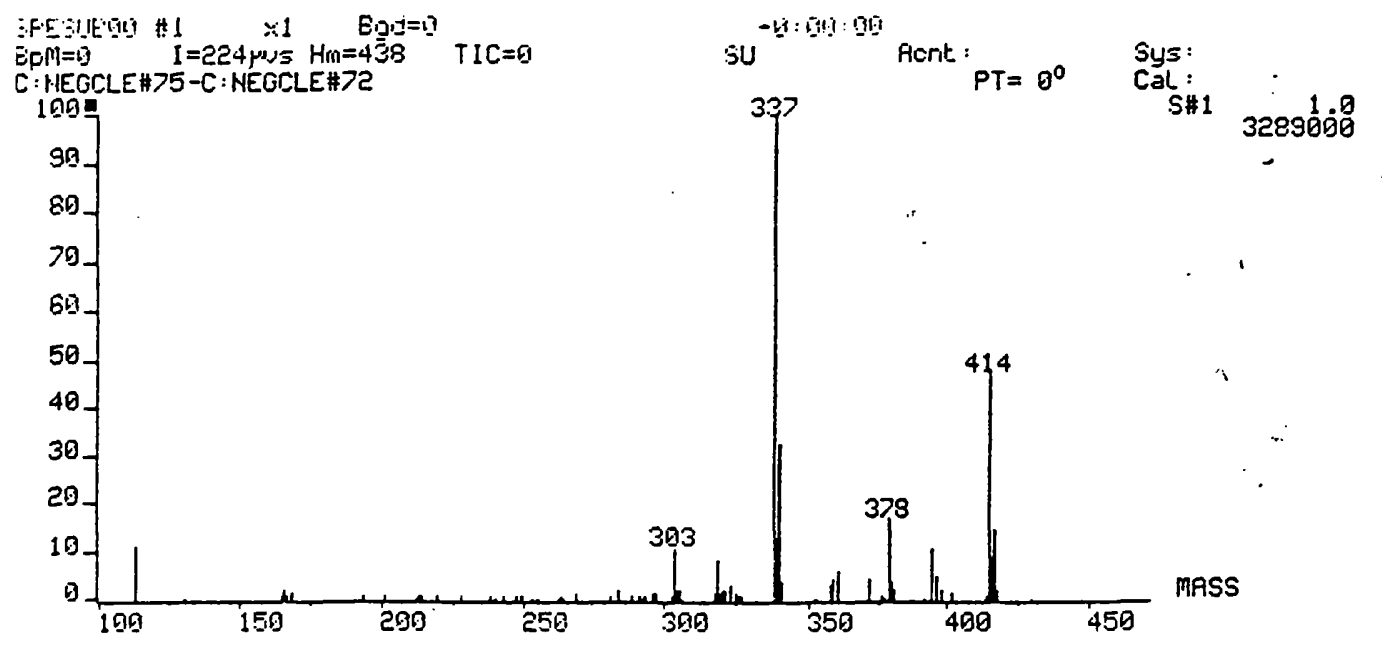

Figure 7.7a Negative Ion Chemical Ionisation Mass spectrum of the TFA derivative of Clenbuterol

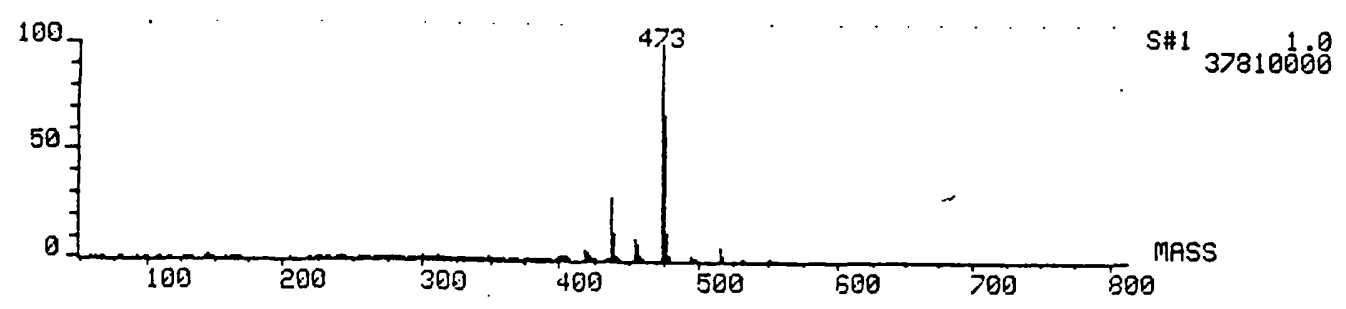

Figure 7.7b Negative Ion Chemical Ionisation Mass spectrum of the PFP derivative of Clenbuterol

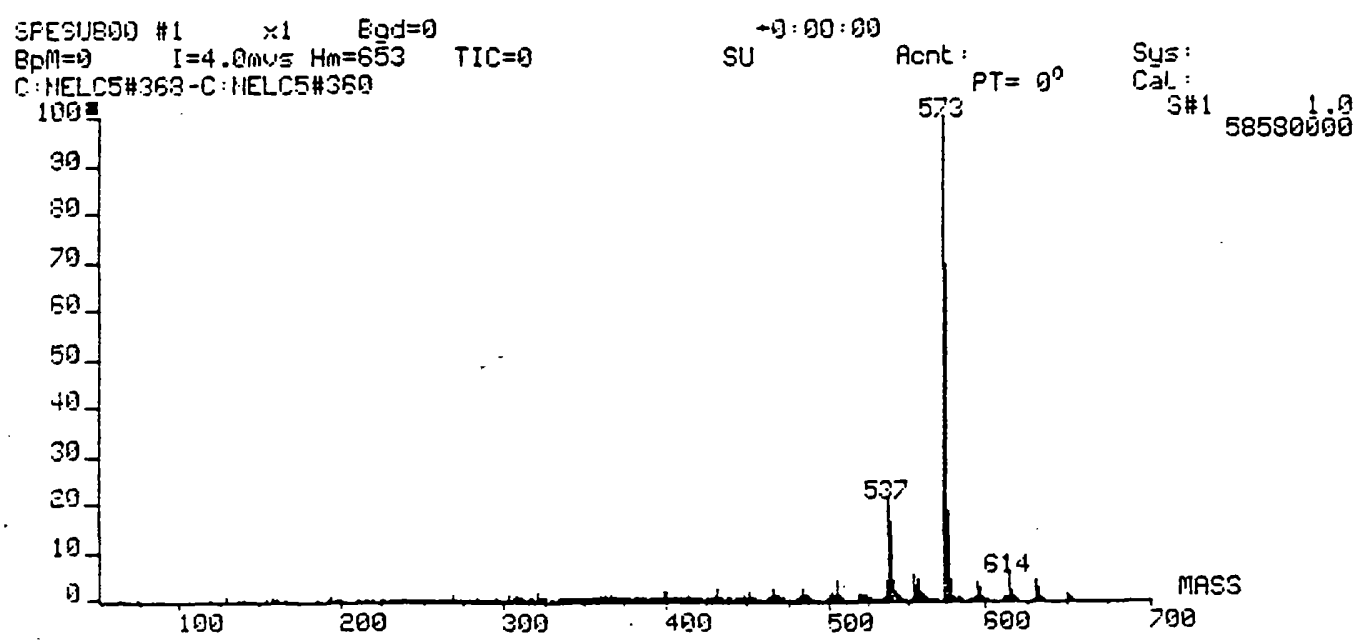

Figure 7.7c Negative Ion Chemical Ionisation Mass spectrum of the HFB derivative of Clenbuterol 
pseudomolecular ion nor the (M-77) ion. The ions corresponding to (M-36) and (M113) losses do exist, however.

The sensitivity of the assay was determined using the HFB derivative under NI-CI conditions. The GC-MS system 1 was operated in the selected ion recording mode at a resolution of 3000 . The ions monitored were the molecular ion $(\mathrm{m} / \mathrm{z} 573$ and $\mathrm{m} / \mathrm{z} 537$ ),. Using these parameters, it was possible to determine the presence of $1 \mathrm{pg}$ of clenbuterol injected onto the column. Any concentrations lower than this resulted in a signal to noise ratio of less than 3:1.

Using NI-CI mass spectrometry in association with HFB derivatives, it was possible to achieve good selectivity and sensitivity. To achieve and maintain this level of sensitivity, however required a disproportionate amount of time for maintenance. When analysis of the samples commenced, it was noted that sufficient sensitivity and specificity could be achieved using GC-MS system 2 in the electron impact mode. All analysis of biological samples were therefore carried out using the alternative GC-MS system 2 in conjunction with TMS derivatisation.

\section{Pharmacokinetic Studies}

Details on dose preparation, animal dosing, sample collection and analysis are given in Chapter 4.

\section{Additional Experimental Details}

Calibration standards were prepared by extraction of standards from urine and plasma. Correlation coefficients for all calibration curves were in excess of 0.99. All samples were analysed in duplicate. Each result had to be within $5 \%$ of the average of the two values.

\subsection{Results and Discussion}

Figures 7.8 and 7.9 show the concentrations of clenbuterol in the plasma of a dog and a bitch respectively versus time after dose. Peak levels for both animals 
appeared to be approximately 5 hours post dose, reaching concentrations of $62 \mathrm{ng} / \mathrm{ml}$ in the dog and 50ng/ml in the bitch. Within 24 hours, plasma concentrations in both animals had fallen to $2 \mathrm{ng} / \mathrm{ml}$. Levels then continued to decline fairly steadily after 24 hours until 168 hours post dose when it was no longer possible to determine the presence of clenbuterol in the plasma of either animal. Between 24 and 168 hours, clenbuterol displayed a half life of approximately 24 hours in the plasma of both animals.

Levels of clenbuterol in urine were, as expected, significantly higher than in plasma. As with the experiments with nandrolone and boldenone, it was not possible to obtain complete urine collections. The results presented in Figure 7.10 and 7.11 show the levels of clenbuterol found per millilitre of urine and per milligram of creatinine for the dog and bitch respectively. When comparing the two animals, a similar urinary excretion pattern was found to exist. When the results were corrected for 'dilution', it can be seen that there was approximately three times the concentration of clenbuterol per milligram of creatinine in the samples obtained from the bitch when compared to the dog (approximately $12.8 \mathrm{ng} / \mathrm{mg}$ creatinine vs. $4.3 \mathrm{ng} / \mathrm{mg}$ creatinine). For both animals, similar amounts of clenbuterol were present in the 24 and 48 hour samples. The male greyhound urine sample had a concentration of $4.3 \mathrm{ng} / \mathrm{ml}$ and $4.4 \mathrm{ng}$ of clenbuterol per $\mathrm{mg}$ creatinine at 254 and 48 hours respectively, falling to $0.5 \mathrm{ng} / \mathrm{mg}$ creatinine by 96 hours. For the female greyhound levels of 12.8 and $11.1 \mathrm{ng} / \mathrm{mg}$ creatinine were present at 24 and 48 hours post dose and this fell to $0.89 \mathrm{ng} / \mathrm{mg}$ creatinine 72 hours. Levels of clenbuterol continued to fall after these initial time periods. It was still possible to determine the presence of clenbuterol 10 days following administration.

Two milligrams of clenbuterol were administered to each animal, giving an approximate dose of $0.0625 \mathrm{mg} / \mathrm{kg}$. Zimmer et al used ${ }^{14} \mathrm{C}$-labelled clenbuterol to dose 5 rabbits and one $11 \mathrm{~kg}$ beagle at $2.5 \mathrm{mg} / \mathrm{kg}$. This level is equivalent to $27.5 \mathrm{mg}$ for an $11 \mathrm{~kg}$ dog or $80 \mathrm{mg}$ for a $32 \mathrm{~kg}$ greyhound. No side effects were reported in either species and the majority of the radioactivity was excreted in the urine by 96 
hours post dose $\mathrm{e}^{92}$. Koyama et $\mathrm{a}^{93}$ also gave up to $2.5 \mathrm{mg} / \mathrm{kg} / \mathrm{day}$ of clenbuterol to beagles with no notable side effects. For comparison Girault et al ${ }^{8}$ administered a dose of $40 \mu \mathrm{g}$ per day to healthy human volunteers and Dumasia and Houghton ${ }^{9}$ administered $800 \mu \mathrm{g}$ daily for four days to horses. Although the dose used in this study appears to be high in comparison to these doses, it was believed to be consistent with the significantly higher doses used by individuals who abuse performance-enhancing substances. 

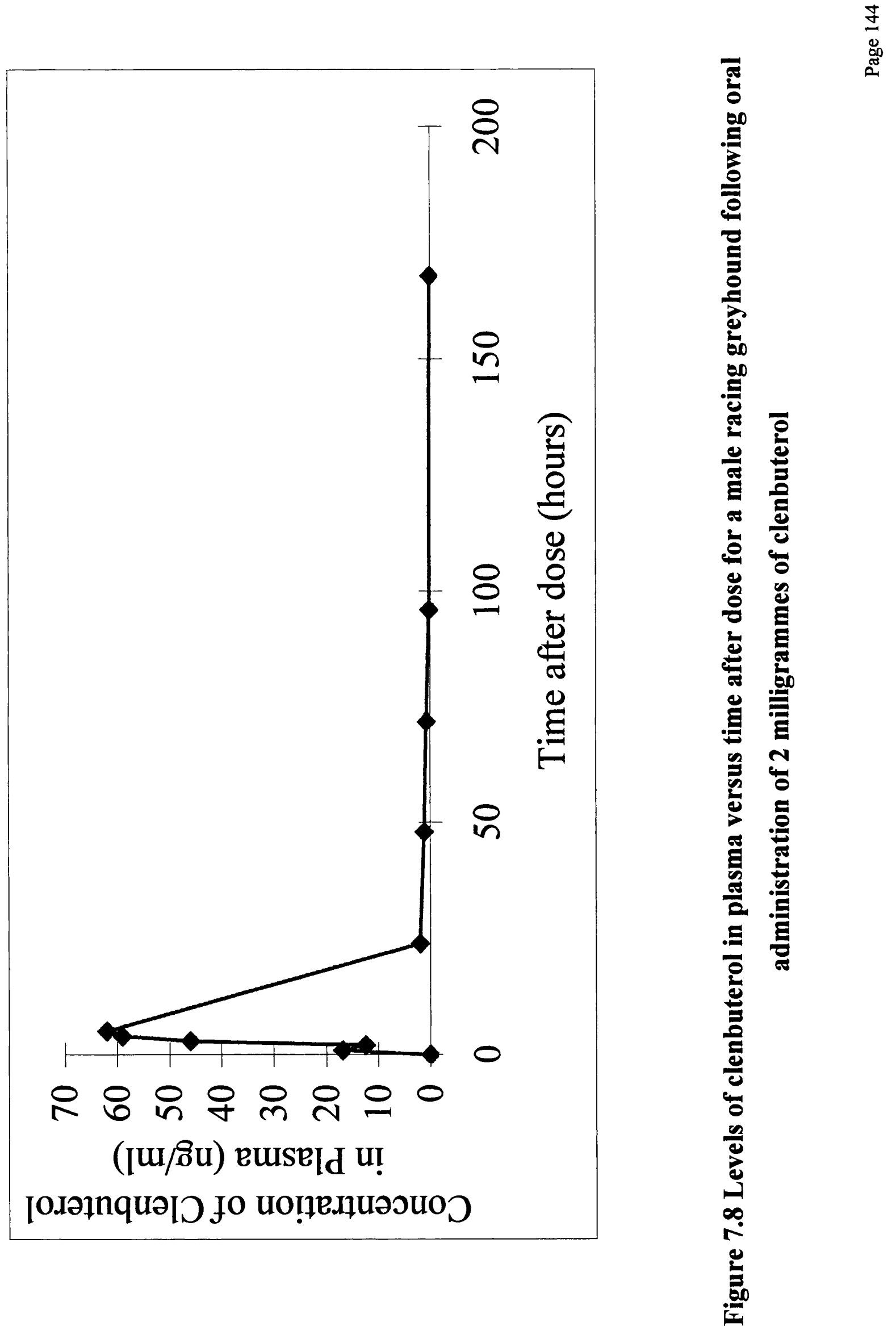


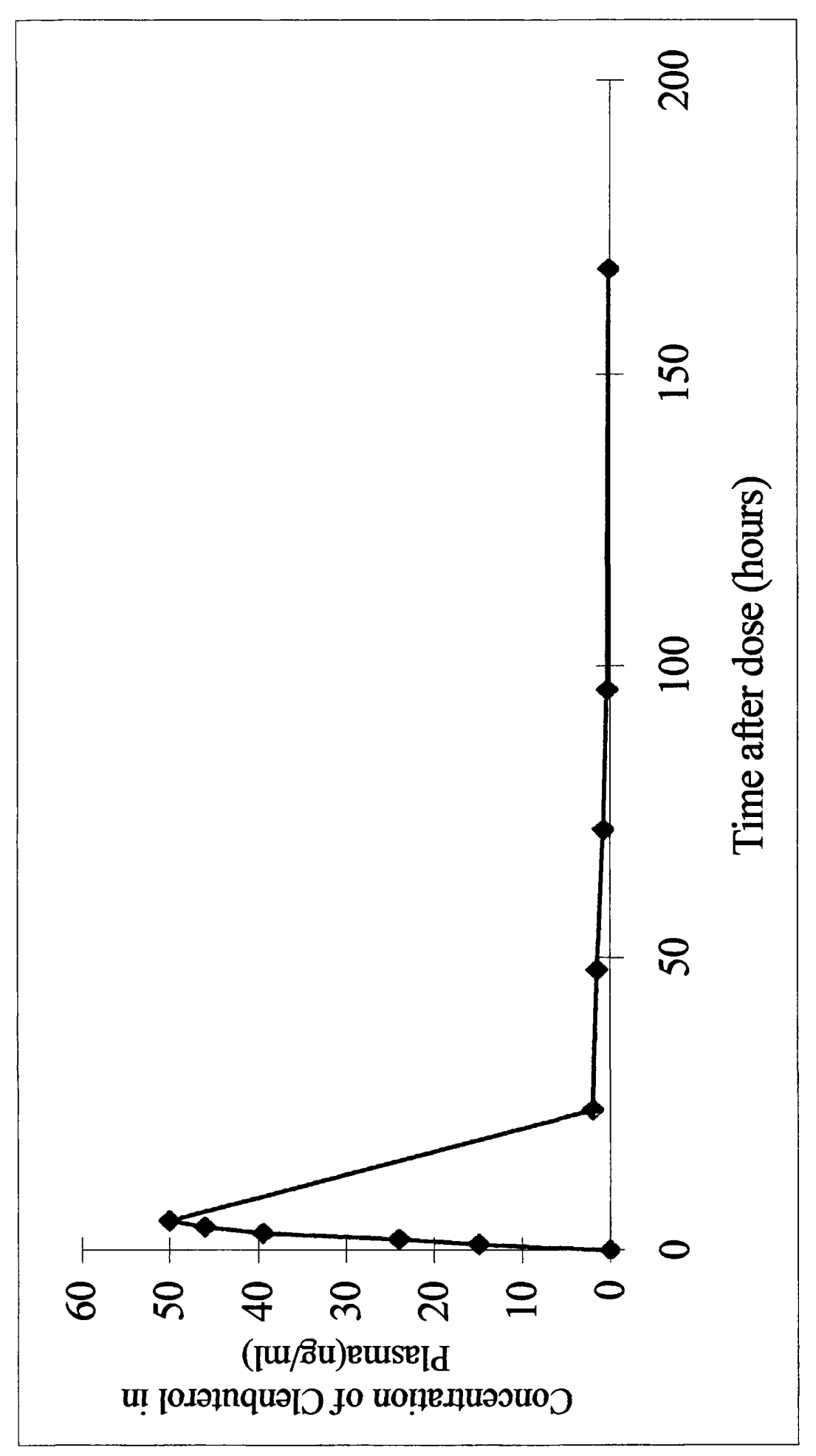

종

훈

.

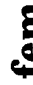

은

s

\&े \&

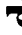

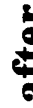

昰

完

눙

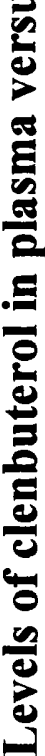

i

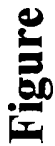




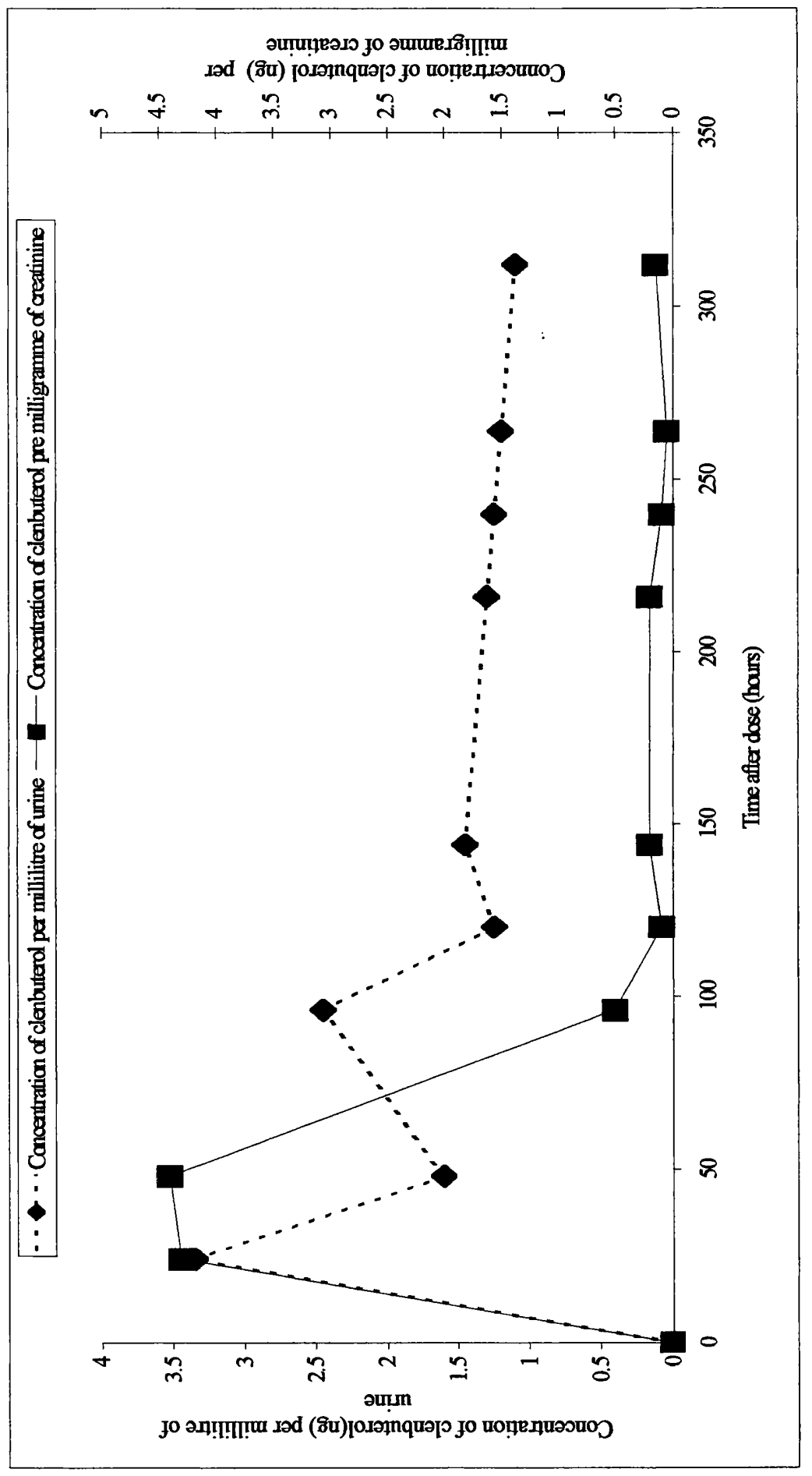

ฐ⿹

를

8

봉

.5

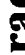

롤

$\rightarrow$ 일

흥 능

\&

过

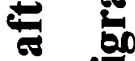

를

ㄴ

응

85

\&

클

I

운

㝵

药 


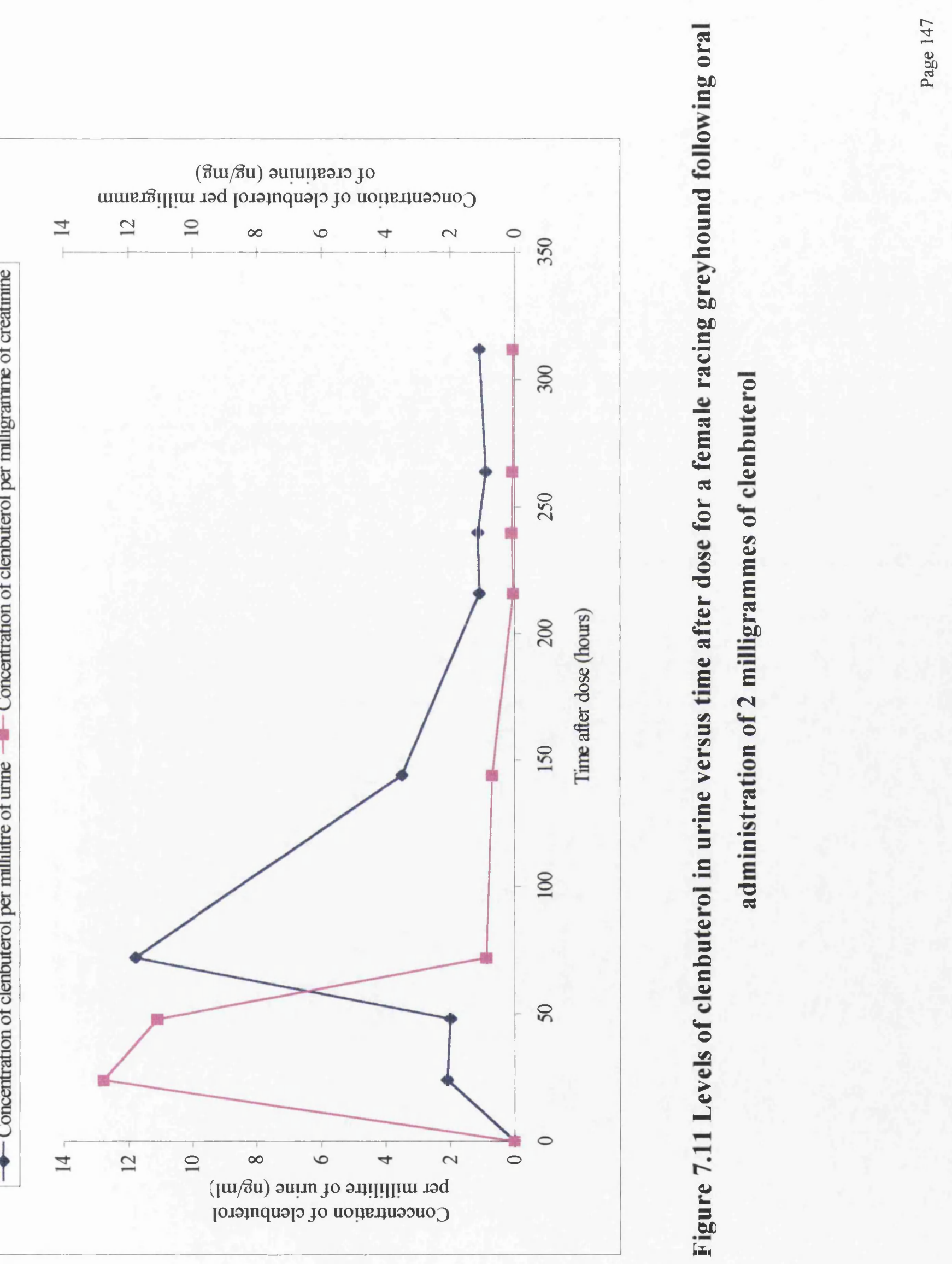




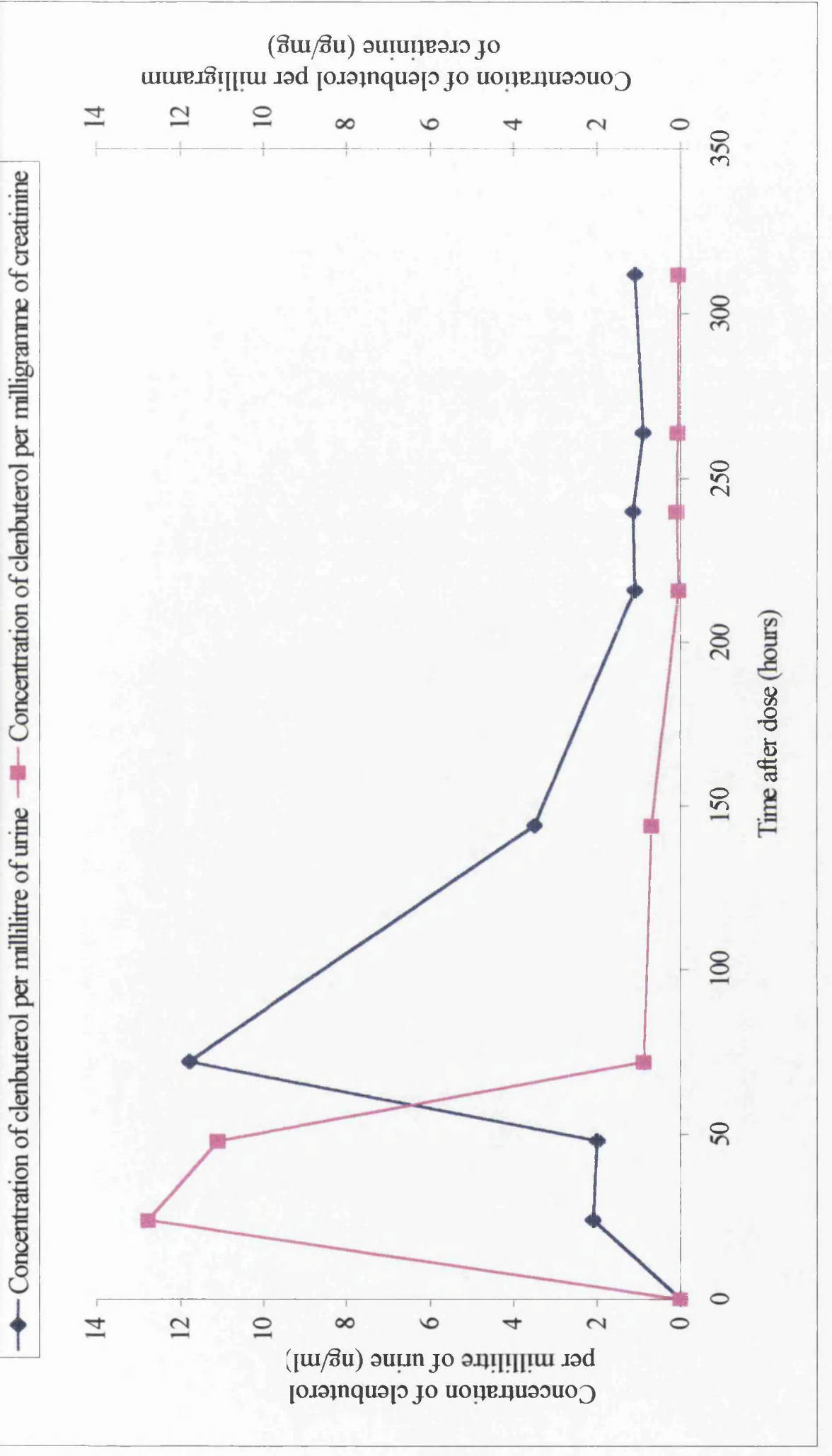


In the greyhounds, however, this dose produced major side effects. Contraindications appeared approximately 2 hours post dose. Heart rate and respiration were both dramatically increased. Both animals became polyuric and polydipsic. Due to the severity of these side effects, blood sampling was suspended 5 hours post dose to avoid any additional stress to the greyhounds. Creatinine levels fell in the urine to such low levels the it was not necessary to perform the normal thirty fold dilution for creatinine analysis, a consequence of the polyurea and polydipsia. Blood sampling was resumed 24 hours post dose and, although dramatically reduced, the side effects were still present. All side effects ceased by 48 hours post dose.

These dramatic side effects may be due to several factors. As indicated earlier, it is not possible to create a $\beta$-agonist which only stimulates a single adrenoceptor sub-type. The symptoms displayed by both greyhounds reflect the stimulation of the adrenoceptors and the activation of the 'flight or fight' response, as reflected by the increased heart rate and respiration. It is possible that the 2 milligram dose was excessive for dogs, which is unlikely as far larger doses were given to beagles with little or no side effects, or that greyhounds may be particularly sensitive to stimulation of the adrenoceptors. In addition, both animals were fully trained race-fit animals with very little body fat. The high volume of distribution of clenbuterol in humans(ca. 350 litres) reflects the distribution of the drug throughout an individual. If the drug was less distributed in the racing greyhound, any side effect could be significantly greater.

The greyhounds used for this metabolism study were trained racing greyhounds. It would be useful to give a reduced dose of clenbuterol to both an actively training greyhound and a 'pet' - an unfit, fat greyhound - to see if the different responses could be attributed to the training regime.

In other breeds of dog, peak plasma levels reached $1.25 \mathrm{ng} / \mathrm{ml} 2$ to 3 hours post administration of a dose of $2.5 \mathrm{mg} / \mathrm{kg}$ and the majority of the dose was excreted from the plasma by 9 hours post dose ${ }^{92}$. As indicated earlier, urine levels were 
almost non-existent 96 hours post dose. In 10 human volunteers, under normal therapeutic dosing conditions, a $20 \mu \mathrm{g}$ dose produced a mean peak plasma level of $86.5 \mathrm{pg} / \mathrm{ml}$ approximately 2.5 hours post oral dose ${ }^{8}$, a similar time to that previously reported for the dog. The half life was reported as 29.5 hours with significant levels of clenbuterol still detected 72 hours post-dose. No data was presented on urine levels. In horses, an $800 \mu \mathrm{g}$ dose for 4 days could be detected in urine using the relatively insensitive technique of full scan electron impact ionisation mass spectrometry up to 96 hours after the final dose ${ }^{9}$. No information was given on plasma levels.

It is well known that it is difficult to extrapolate pharmacokinetic data between species. In these studies there appear to be significant intra-species variation as well as inter-species similarities. In the beagle, two studies revealed little or no side effects at significantly higher doses than those used in this study. This level in greyhounds produced significant side effects. The half-life of clenbuterol in humans and greyhounds appears to be similar at 29.5 and 24 hours respectively. No information is available regarding the plasma half-life of clenbuterol in either the beagle or the horse. Due to the side effects of the dose, it was not possible to determine the exact time for peak plasma concentration in the racing greyhound due to the interruption of the plasma sampling schedule.

However, it does appear to be at least 5 hours post dose, significantly longer than in either the beagle or human. The pattern of urinary excretion is similar in both dog breeds, with the majority excreted by 48 hours post administration. It would appear that an extended period of elimination exists in the greyhound and possibly the horse, as it is possible to detect the presence of clenbuterol by full scan electron impact mass spectrometry up to 96 hours post dose.

The longer detection time of clenbuterol in urine samples of the greyhounds when compared to horses may be due to the larger dose of clenbuterol given to the greyhounds and the relatively small volume of urine eliminated by the dogs, effectively concentrating the urine sample. 
In conclusion, following a single, oral 2 milligram dose of clenbuterol it is possible to detect the drug in the plasma of a racing greyhound for up to 7 days and in the urine for up to ten days. The two milligram dose produced significant side effects in actively trained racing greyhounds, which had not been suggested by previous studies in beagles. 


\section{Chapter 8 Conclusions}

This project was designed to examine the metabolism of anabolic agents in the racing greyhound. Six targets were set and each of them can be dealt with separately.

To establish a metabolic unit for greyhounds in which dogs would be trained in an identical manner to racing animals.

Previous projects within the Department of Forensic Medicine and Science, University of Glasgow have always used greyhounds that were effectively pet dogs and kept at the Veterinary Medicine School. These animals were caged and used to being handled. They were, however, quite old animals and had been used for multiple metabolism experiments. Consequently, the collection of samples from these animals has been relatively easy and it was possible to keep the greyhounds in metacages that would permit total urine collections. In addition, for serial sampling, indwelling cannulae could be used. This reduced significantly the amount of damage caused by repeated venipuncture.

In the present work, only the radiolabelled-testosterone dosing experiment used a captive animal in a metacage, and that was for health and safety reasons. Safety regulations would have been breeched if radioactive urine and faeces were allowed to contaminate the kennel environment.

The aim of using actively training greyhounds was to determine the metabolic profiles of actual racing animals and to determine if the use of anabolic agents could in reality improve the track times of the individual animals. Subtle differences may 
exist between trained and untrained animals. The sampling regime however, which involved venepuncture, damaged the animals and on the advice of the trainer the animals were not time trialed. The trainer felt that it was possible that more serious injury could result if these animals were trialed. When the animals were not being used for the metabolism experiments, however, they were treated exactly as the other greyhounds prepared for racing by the trainer.

The greyhounds were kennelled approximately 60 miles from the Department and the metabolism experiments were carried out under Home Office Licence at the kennels. These settings were not ideal. As stated earlier, it was not possible to obtain complete urine collections. This would have been advantageous and may have allowed for a more detailed determination of the elimination profiles of the greyhounds. When blood samples were collected, it was not always possible to separate the plasma from the red blood cells as quickly as would be preferred. Some lysis of the red blood cells could occur. A small centrifuge was supplied to the kennels to overcome this problem. A local veterinary nurse was required to come and collect the blood samples. Due to her working pattern, it was not always possible to collect all the desired samples, especially samples times that occurred over the weekend. In a more controlled environment a more appropriate sampling protocol could have been employed, although it may not have been possible to train the greyhounds.

In spite of these problems, it was possible to use the greyhounds for the metabolism experiments in the training environment. It may have been interesting, however to have compared the metabolic profiles between the racing greyhounds and the 'pet' to determine if there were any major differences caused by the training regime. 
To characterise the normal urinary and plasma steroid profiles for the racing greyhound.

To summarise, the levels of steroids present in the plasma of the male and female racing greyhound appear to be extremely low. Unlike humans, it was not possible to determine a normal urinary or plasma steroidal profile using full scan mass spectrometry in the male racing greyhound. It was possible, however, to detect the presence of some steroids in the female. The presence of these steroids in the female may be an indicator of the onset of oestrus. Using selected ion monitoring, it was possible to determine the presence of some steroids (testosterone, epitestosterone), but at levels of a few nanogrammes per millilitre. Levels of testosterone determined by radioimmunoassay corroborated these mass spectrometry findings. Therefor, unlike humans, greyhounds do not appear to have significant amounts of steroids in their plasma or urine.

To obtain some basic pharmacokinetic information regarding steroid metabolism in the racing greyhound.

Using labelled forms of testosterone it was possible to determine some pharmacokinetic information from male greyhounds. It appears that testosterone is rapidly removed from circulation following the intravenous dosing of a small amount of radiolabelled testosterone. It also appears that enterohepatic recirculation occurs, suggesting that at least some of the testosterone is eliminated via the bile. Only $40 \%$ of the radioactivity was recovered in the urine up to 7 days after dosing and this level seems to have plateaued. This suggests that up to $60 \%$ of the dose is eliminated via the faeces. Administration of deuterium labelled testosterone to a 
greyhound gave a dilution factor which suggested a pool size for testosterone of approximately $190 \mu \mathrm{g}$. This pool size is between 5 and 6 times that of a human male and appears to be disproportionaly large. It may be that the administration of such a large deuterium labelled dose totally disrupted the steroid metabolism of the greyhound and that this pool size should be regarded with caution. If such a large pool of testosterone existed, it would be expected that some metabolites of testosterone could be detected in urine samples. As discussed earlier, however, there were little or no steroids detected in the urine samples analysed.

\section{To examine the pharmacokinetics of specific anabolic steroids in the racing greyhound.}

Nandrolone and boldenone were given to two male racing greyhounds and the urine was analysed for the presence of the parent compounds. It was noted that nandrolone appeared to be present prior to the doping of the animals, suggesting that nandrolone is in fact an endogenous steroid to the racing greyhound. This is consistent with findings in other species ${ }^{89,90}$. The concentration of nandrolone found in the urine was extremely low. The lack of boldenone in the urine sample along with the low concentrations of nandrolone suggest that the steroids are extensively metabolised prior to excretion, or else, like testosterone, are excreted in faeces. In one of the few papers dealing with steroids and the racing greyhound, Brockwell and co-workers had similar findings, including the presence of testosterone for only two days after dosing with testosterone. ${ }^{72}$ 
To examine the effects of exogenous steroids on the natural steroid profile of the racing greyhound.

The administration of nandrolone and deuterium labelled testosterone appeared to depress the levels of steroids found in plasma and urine samples. During the nandrolone experiment, the steroid profile of one greyhound appeared to be completely eliminated. This loss was probably caused by the negative feedback loops within the pituitary-hypothalamus-gonadal axis of the animals. In addition to the suppression of the steroid levels there appeared to be a suppression of the creatinine eliminated from the body as the nandrolone exerted its anticatabolic effect. The elimination of creatinine appeared to return to normal after approximately 10 to 12 days. While this may be an inexpensive way to determine the administration of an anabolic steroid to an individual animal, it is likely to be unspecific and of little practical use when the normal ranges of creatinine for the greyhound are established. Boldenone appeared to have a significantly lower effect on the steroid profile of the racing animals, possibly due to the lower does applied. The use of internal markers to determine anabolic steroid use, such as the T:E ratio was unsuccessful in these studies but recent publications suggest that internal markers can be used to determine the exogenous administration of endogenous steroids such as testosterone $e^{94}$. 


\section{To examine the pharmacokinetics of other anabolic agents in the racing}

greyhound

Clenbuterol was chosen as a model non-steroidal anabolic agent. The dose given to one male and one female greyhound caused very severe side effects. It was possible however to determine the presence of clenbuterol in the plasma and urine sample for an extended time period (at least 10 days) post dose. The clinical indications corresponded to the known side effects of beta agonists.

\section{Future Work}

It does appear that there are some unique features regarding the metabolism of steroids and other anabolic agents in the racing greyhound. These features may be applicable to the canine species in general or may be applicable specifically to the greyhound. These features may arise as a result of persistent inbreeding to create the current form of racing greyhound. As a consequence of inbreeding other species of dog have acquired genetic traits. Dalmatians, for example, are prone to be deaf at birth and have chronic kidney problems. It may be that the lack of steroids in the urine and the sensitivity of greyhound to beta agonists are as a result of genetic traits. It is also possible that the differing responses may be due to the training regime employed.

Consequently, it would be appropriate to investigate the steroid profile and metabolism of anabolic steroids and anabolic agents both in untrained greyhounds 
and other species. This would help resolve the issues surrounding particular susceptibilities to certain drugs.

In future studies of greyhound steroid biochemistry, more sensitive methods would be required than those available for the work presented in this thesis, and this is likely to be possible if newer instrumentation were to be used. Also, the present study provided some indications that steroids are excreted via bile into the faeces. Other drugs are known to be excreted in faeces in the dog, for example non-steroidal anti-inflammatory drugs ${ }^{95}$. Future pharmacokinetic studies on the greyhound should incorporate faecal analysis as part of the study plan for this reason. The disturbances to the normal greyhound steroid biosynthesis noted in the present work require further investigation, to elicit the factors controlling endogenous synthesis and how these are disrupted in the greyhound by administration of anabolic steroids. This would be essential if screening methods similar to those used in the human for steroid doping are to be developed. The analytical scheme for future studies should therefor include peptide hormones.

Although two anabolic steroids and one beta agonist have been studied here, it would be appropriate to extend the range of compounds studied. Suggested compounds include estradiol, zeranol, stanozolol and other beta agonists. If additional studies were to be carried out, it would be easier to use a combination of unlabelled, deuterium labelled and radiotracer material simultaneously. This was not possible in the present study, as the aim was to use actively trained racing greyhounds

Overall, the aims of the study were met, and provide starting points for future work. 


\section{Acknowledgements}

I wish to thank all the people were involved in this project form conception through to delivery but some deserve a specific mention.

First, I would like to thank the National Greyhound Racing Club for providing the financial support for this project and Professor Hamilton Smith for allowing me to carry out the work in his Department.

I owe a huge debt to Dr Robert Anderson of the Department of Forensic Medicine and Science, University of Glasgow for supervising this work, all his suggestions, support and encouragement. Thanks for all your efforts Bob.

The animal work was carried our with Mr John Spence, greyhound trainer, Marie the nurse, Edward Boyce and Michael Boyce, and the Kennel girls at the Vet school. Thanks to all of you.

To Patrick, a friend and colleague, thanks.

To Max, Edgar, Blue, Black and White 1, Black and White 2 and the Brindle, thank you.

To the staff at the Department for your help and friendship

Finally, to all my family and friends who put up with me talking about urine for a long time, thanks. 


\section{References}

1 Daniel F. Hanley in Sports Medicine and Physiology, R.H. Strauss (ed.) Saunders, 1979.

2 Les Woodland, 'Dope, The use of drugs in Sport', p 54, 1980, David \& Charles, Newton Abbot and London

3 Les Woodland, 'Dope, The use of drugs in Sport', p 55, 1980, David \& Charles, Newton Abbot and London

4 WW Franke, B Berendonk, 'Hormonal doping and androgenisation of athletes: a secret programme of the German Democratic Republic Government', Clin Chem, 43(1997), 1262-1279

5 International Olympic Committee medical code, Internet address http://www.nodoping.org/medch2_e.html\#anaboli

6 KM Hold, DG Wilkins, DJ Crouch, DE Rollins, RA Maes 'Detection of stanozolol in hair by negative ion chemical ionization mass spectrometry', J Anal Toxicol, 20(1996), 345-49

7 Daniel Duchaine 'Underground Steroid Handbook (11) Update :1992', first printing

8 J Girault, P Gobin, J B Fourtillan, 'Quantitative measurement of Clenbuterol at the femtomole level in plasma and urine by gas chromatography/negative ion chemical ionisation mass spectrometry', Biomed Environ Mass Spectrom, 19 (1990), 80-88

9 MC Dumasia, E Houghtan, 'Screening and confirmatory analysis of $\beta$ agonists, $\beta$-antagonists and their metabolites in horse urine by capillary gas chromatography mass spectrometry.' J Chrom., 564 (1991) 503-513 
10 Steven Karch, 'The Pathology of Drug Abuse’, p357, 1993, CRC Press Inc, Boco Rotan, Florida

11 From 'Rules of Racing and directions of the Stewards of the National Greyhound Racing Club Ltd', Published by Stewards of the National Greyhound Racing Club Ltd, October 1989, Gabare Ltd, London and Winchester

12 IUPAC-IUB Revised tentative Rules for Steroid Nomenclature copyright (1969) AM. Chem. Soc., published Biochemistry. 8, (1969), 2227 and Biochem J., 113, (1969), 5

13 Definative rules for the nomenclature of steroids, Pure Applied Chem., 31, (1972), 85

14 Richard Palmiter in 'Biochemistry', p 1104, G Zubay (Ed.), 1984, AddisonWesley Publishing Inc, Reading Massachusetts

15 J. Gorski, D.O. Toft, G. Shyamala, P Smith, A. Notides, 'Hormone Receptors: Studies on the interaction of estrogen with the utereus', Recent Prog. Horm. Res., 24 (1968), 45-80.

16 E.V. Jensen, T. Suzuki, T. Kawashima, E.W. Stumpf, P.W. Jungblut, E.R. de Sombrone, 'A two step mechanism for the interaction of estradiol with rat uterus', Proc. Natl. Acad. Sci. U.S.A, 59(1968), 632-638.

17 B.S. Katzenellenbogen, 'Dynamics of steroid hormone receptor action' Ann. Rev. Physiol., 42(1980), 17-35.

18 V.K. Moudgil, (ed) 1987, Recent Advances in Steroid Hormone Action, Walter de Gruyle and Co., New York. 
19 O.M. Conneely, W.P. Sullivan, D.O. Toft, M. Birnbaumer, R. Cook, B. Maxwell, T. Zarucki-Schultz, G. Greene, W. Schrader, B. O'Malley, 'Molecular cloning of the chicked progesteron receptor', Science, 233(1986), 767-770. 20 P. Walter, S. Green, G. Greene, A. Krust, J.-M. Bornert, J.-M. Jeltsch, A. Staub, E. Jensen, G. Scrace, M. Waterfield, P. Chambon, 'Cloning of the human estrogen receptor cDNA', Proc. Natl.Acad. Sci. U.S.A., 82(1985), 7889-7893.

21 H. Loosfelt, M. Atger, M. Misrahi, A. Guiochon-Mantel, C. Meriel, F. Logeat, R. Benarous, E. Milgrom, 'Cloning and Sequence analysis of Rabbit progesterone-receptor complementory DNA', Proc. Natl. Acad. Sci. U.S.A., 83(1986), 9045-9049.

22 M. Danielsen, J.P. Northrop, G.M. Ringold, 'The mouse glucocorticoid receptor: mapping of functional domains by cloning, sequncing and expression of wild-type and mutant receptor proteins', Embo. J., 5(1986),2513-2522.

23 S. M. Hollenberg, C, Weinberger, E.S. Ong, G Carelli, A. Oro, R. Lebo, E Thompson, R. M. Evans, 'Primary Structure and expression of a functional human glucocorticoid receptor cDNA', Nature, 318(1985), 635-641.

24 R. Miesfeld, S. Rusconi, P.J. Godowski, B.A. Maler, S. Okret, A.-C. Wikstrom, J.A. Gustafsson, K.R. Yamamoto, Cell, 'Genetic complementaion of a glucocorticoid receptor deficiency by expression of cloned receptor cDNA', 46(1986), 389-399.

25 A. Krust, S. Green, P. Argos, V. Kumar, P. Walter, J.-M. Bornert, P. Chambon, 'The chicken oestrogen receptor sequence, homology with v-erbA and the human oestrogen and glucocorticoid receptors', EMBO J., 5(1986), 891-897. 
receptor:sequnce homology with the human and avian receptors and identification of multiple estrogen receptor messenger ribonucleic acids', Mol. Endocrinol., 1(1987), $355-362$.

27 S. Green, V. Kumar, A. Krust, P. Chambon, in Recent Advances in Steroid Hormone Action, V.K. Moudgil, (ed) (1987), 161-183 Walter de Gruyle and Co., New York.

28 R. White, J.A. Lees, M. Needham, J. Ham, M. Parker, 'Structural organisation and expression of the mouse estrogen receptor', Mol. Endocrinol, 1(1987), 735-744.

29 F. Pakdel, C. Le Guellec, C. Vaillant, M.G.L. Roux, Y. Valotaire, 'Identification and estrogen induction of two estrogen receptors (ER) messenger ribonucleic acids in the rainbow trout liver: sequence homology with other Ers', Mol Endocrinol, 3(1989), 44-51.

30 R. Hume, G.S. Boyd, 'Cholesterol metabolism and steroid hormone production', Biochem Soc. Trans., 6(1978), 893898

31] E.R. Simpson, Mol. Cell Endocr., 13, (1979), 213

32 A.M. Neville, J.L Webb, T. Symmyton, 'The in vitro utuliazation of (4-14C)dehydroisoandrosterone by human adrenocortical tumors associated with virilism, Steroids, 13(1969), 821

33 I. Welikg, L.L Engle, 'J, Biol. Chem., 238, (1963), 1302

34 A. Vermeulan, 'The hormonal activity of the postmenopausal ovary', J. Clin. Endocr. Metab., 42(1976), 247-253 
35 C. Longcope, W Widrich, C.T. Swain, 'The secretion of estraone and estradiol by human testis', Steroids, 20,(1972), 439-448

36 J. Goto, J. Fishman, 'Participation of a nonenzymatic transfornation in the biosynthesis of estrogens from androgens', Science, 195(1977), 80-81

37 W.G. Kelly, O. Devlan, T.H. Rizkallah, 'The role of 19-hydroxy-delta4androstene-3,17-dione in the conversion of circulating delta4-androstene-3,17 dione to estrone', J. Clin. Endocrinol. Metab, 43(1976), 190-194

38 F.L. Bellino, Y. Osawa, ' Solubilisation of estrogen synthetase from human terme placental microsomes using detergents', J Steroid Biochem, 9(1978),219-228 39 E.A. Thompson, P.K. Siiteri, 'Partial resolution of the placental microsomal aromatase complex', J. Ster. Biochem., 7(1976), 635-639

40 L.D. Garren, G.N. Gill, H. Masin, G.M. Walton, 'On the mechanism of action of ACTH', Rec. Prog. Horm. Res.,27(1971),433-478

41 G.S. Boyd, A.M.S. Gorban, in Recently Discovered Systems of Enzyme Regulation by Reversible Phosphorylation', ed. P. Cohen, Elsevier/North Holland Biomedical Press, Amsterdam.

42 C.T. Privalle, J.F. Crivello, C.R. Jefcoate, 'Regulation of intramitochondrial cholesterol transfer to side-chain cleavage cytochrome P-450 in rat adrenal gland', Proc. Natl. Acad. Sci. USA, 80(1983), 702-706

43 W. Vale, J. Spiess, C. Rivier, J. Rivier, 'Characterisation of a 41 residue ovine hypothalmic peptide that stimulates secretion of corticotrophin and beta endorphin',Science, 213(1981), 1394-1397

44 W.Y. Huang, R.C.C Chang, T.N. Redding, S. Vigh, A.V.Schally, Fed. Proc., 41, (1982), 1458 
45 A. Grossman, A.C. Niewenhuyzen Kruseman, L. Perry, S. Tomlins, A.V.

Schally, D.H. Coy, L.H. Rees, A.M. Comaru-Schally, G.M. Besser, 'New hypothalmic hormome corticotrophin-releasing factor, specifically stimulates the release of adrenocorticotrophin hormone and cortisol in man', Lancet, 1982, 921922

46 S.J. Main, R.V. Davis, B.P. Setchell, 'Effects of passive immunisation against testosterone on serum aand pituitary gonadotrophins: implications for the relative inportance of androgens and inhibin in the control of gonadotrophin secretion in the male rat', J. Endocrinol., 86(1980), 135-146

47 R.V. Brooks, Biochem. J., 68, (1958), 50

48 A.E. Kellie, E.R. Smith, Biochem. J., 66, (1957), 490

49 DB Gower and JW Honor in 'Principles of Steroid Biochemistry', p365, HLJ Makin (Ed.), 2nd Edition, 1984, Blackwell Scientific Publications, Oxford 50 DB Gower and JW Honor in 'Principles of Steroid Biochemistry', p365, HLJ Makin (Ed.), 2nd Edition, 1984, Blackwell Scientific Publications, Oxford 51 DB Gower in 'Principles of Steroid Biochemistry', p280-282, HLJ Makin (Ed.), 2nd Edition, 1984, Blackwell Scientific Publications, Oxford 52 DB Gower and JW Honor in 'Principles of Steroid Biochemistry', p384, HLJ Makin (Ed.), 2nd Edition, 1984, Blackwell Scientific Publications, Oxford 53DB Gower and JW Honor in 'Principles of Steroid Biochemistry', p383-387, HLJ Makin (Ed.), 2nd Edition, 1984, Blackwell Scientific Publications, Oxford 54 E Nieschlag, H Behre in 'Testosterone, action, deficiency substitution', E Nieschlag and H Behre (Eds), 1990, Springer-Verlag, Berlin 
M C Dumasia, E Houghton, 'Biotransformation Of 1-Dehydrotestosterone In

The Equine Male Castrate: Identification Of The Neutral Unconjugated And Glucuronic Acid Conjugated Metabolites In Horse Urine', Biomed. Environ Mass Spectrom, 17(1988), 383-392.

56 C Y Gradeen, S C Chan, P S Przybylski, 'Urinary excretion of furazebol metabolite', J Anal Toxicol, 14(1990), 120-122

57 R Masse, H Bi, C Ayotte, P Du H Gelinus, R Dugal, Studies on anabolic steroids. V. Sequential reduction of methandienone and structurally related A-ring substituents in humans: gas chromatographic-mass spectrometric study of the corresponding urinary metabolites', J Chrom, 562(1991), 323-340

58 W Schanzer, H Geyer, M Donike, 'Metabolism of metandienone in mad: identification and synthesis of conjugated excreted urinary metabolite, determination of excretion arates and gas chromatographic -massspetrometric identification of bishydroxylated metabolites', J Steroid Biochem Mol Biol, 38(1991), 441-464

59 D Goudreault R Masse, 'Studies on anabolic steroids 4. Identification of new urinary metabolites of methenolone acetate (primobolan) in human by gas chromatography-mass spectrometry', J Steroid Biochem Molec Biol, 37(1990), 137154

60 L A van Ginkel, R W Stephany, H J vna Rossum, H van Blitterswijk, P W Zoontjes, RCMHooischuur, J Zuydendorp, 'Effective monitoring of residues of nortestosterone and its major metabolites in bovine urine and bile', J Chrom, 489(1989), 95-104

61 E Houghton, G A Oxley, M S Moss. S Evans, 'Studies related to the metabolism of anabolic steroids in the horse: a gas chromatographic mass 
spectrometric method to confirm the administration of 19-nortestosterone or its ester to horses', Biomed Environ Mass Spectrom, 5(1978), 170-173

62 R Masse, H Bi C Ayotte, R Dugal, Biomed Environ Mass Spectrom, 18 429438

63 R Masse, C Ayotte, H Bi R Dugal. J Chrom 497 (1989) 17-37

64 W Schanzer, G Opfermann, M Donike, J Steroid Biochem (36(1990)153-174

65 W M Muck, J D Henion Biomed Environ Mass Spectrom, 19 (1990)37-51

66 A.G.Rico V Burgat Sacaze, Curr Top Vet Med Anim Sci, 26, (1984) 72-84

67 W Schanzer, M Donike, 'Metbolism of anabolic steroids in man: the synthesis and use of reference material for the identification of anabolic steroid metabolites', Anal Chim Acta, 275(1993), 23-48

68 Steven Karch, ‘The Pathology of Drug Abuse’, p357, 1993, CRC Press Inc, Boco Rotan, Florida

69 Martindale,

70 M Richkind and L-E Edqvist, Peripheral plasma levels of corticosteroids in normal beagles and greyhounds measured by a rapid competitive binding technique, Acta Vet Scand., 1975, 745-757Richkint and Enqqvist, Acta Vet Scan, 1975, 14, $745-757$

71 Paul L Pemberton, 'The use of anabolic steroids and vitamins in the racing Greyhound.', 34 191-198

72 M Brockwell, J Knox, A Stenhouse and J Ralston, 'The identification of the metabolites of testosterone, 19-nortestosterone and 1-dehydrotestosterone in Greyhound Urine' from Proceedings of the Ninth International Conference of Racing Analysts and Veterinarians,' Volume 1 Analytical Topics, Ed. Charles R 
Short, Pub The International Conference of Racing analysts and Veterinarians, 1992

Baton Rouge.

73 J Ralston, S Ngo and A Stenhouse 'Detection of 19-nortestosterone in Equine and Greyhound Urine' from Proceedings of the Ninth International Conference of Racing Analysts and Veterinarians,' Volume 1 Analytical Topics, Ed. Charles R Short, Pub The International Conference of Racing analysts and Veterinarians, 1992 Baton Rouge.

74 J Chapman in 'Practical Organic Mass Spectrometry', 2nd Edition, 1993, John Wiley and Sons, Chichester

75 'Sorbent Extraction Technology Handbook'. Eds. N Simpson and K C Vab Horne, Varian Sample Preparation Products, Harbor City, California.

76 C.H.L. Shackleton and J.W. Honour, Clin. Chim. Acta, 69 (1976) 267.

77 C.H.L. Shackleton and J.O. Whitney, Clin. Chim. Acta, 107 (1980) 231.

78 B. Green and R.E. Leake, (Eds.), Steroid Hormones, A Practical Approach, IRL Press, Oxford, 1987, p. 54.

79 A. Beelinger, J. Couture, S. Coron and R. Roy, Ann. N. Y. Acad. Sci., 595 (1989) 251.

80 H. Ehrsson, T. Walle and H. Brotell, Acta Pharmaceutica Suicica, 8 (1971) 319.

81 Sigma Diagnostics Creatinine Kit, Procedure No. 555

82 S Biddle, Personal communication, 1999

83 Walter Jennings, personal communication, November 1990

84 Dr. R Heitzman, Personal communication 1998

85 Dr R Heitzman, Personal communication, 1999 
86 ABPI compendium of data sheets for veterinary products 1985-1986 pp445-446.

87 ABPI compendium of data sheets for veterinary products 1985-1986, pp120.

88 M Donike, , H Geyer, M Kraft and S. Raouth in P. Billotti, G Benzi and A Ljungquist (Eds.) Proceedings of 2nd IAF World symposium on Doping in Sport, Monte Carlo, 5-7 June 1989, International Athletic Foundation, Rome 1990, P107. 89 Ed Houghtan, 'Anabolic steroids in the horse-a review of current knowledge' from proceedings of the ninth international conference of racing analysts and veterinarians,' Volume 1 Analytical Topics, Eds Charles R Short, Pub The International Conference of Racing analysts and Veterinarians, 1992 Baton Rouge. 90 G Kennedy , Personal communication.

91 L Leyssene, C Driessen, A Jacobs, J Chech, J Raus, 'Deteriminations of $\beta 2$ receptor agonists in bovine urine and liver by gas chromatography tendem mass spectrometry.', J Chrom., 564 (1991) 515-527

92 VA Zimmer, A Bucheler, S Kaschke, 'Pharmakokinetik und Metabolitenmuster von clenbuterol beim kaninchen und beim hund.', Arzneim Forsch, $26(1976) 1442-5$

93 T Koyama, A Nagami, H Uno, T Makita, S Seki, A Inoue, K Ojima, M Enomoto, 'Toxicity Studies of Clenbuterol (V); Chronic toxicity study of clenbuterol in dogs', Iyakuhin Kenkyu, 15(2) 1984, 855-905

94 V Ferchard, B Le Bizec, F Monteau, F Andre, 'Determination of the exogenous character of testosterone in bovine urien by gas chromatography-combustion-isotope ratio mass spectrometry', Analyst, 123(1998), 2617-2620

95 A Study of Non-steroidal Anti-inflammatory Drugs in the Urine of the Racing Greyhound, Ph.D. Thesis, University of Glasgow, 1990 